LBL--20324

DE86 002870

Search for Right-Handed Currents

by Means of Muon Spin Rotation

David Philip Stoker

Ph.D. Thesis

Lawrence Berkeley Laboratory

University of California.

Berkeley, California 94720

September 1985

\title{
DISCLAIMER
}

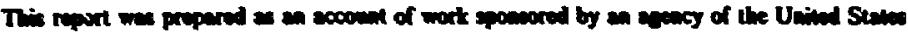

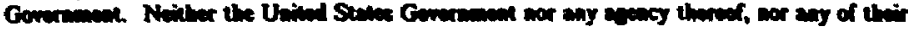

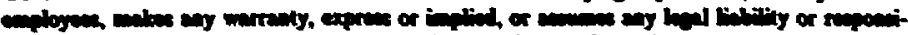

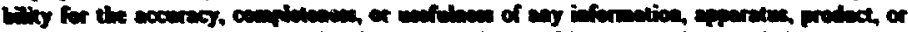

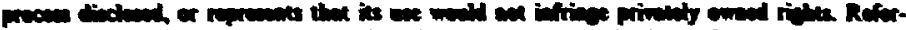

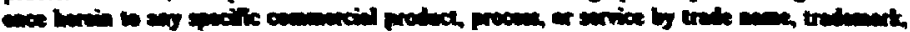

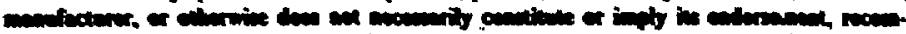

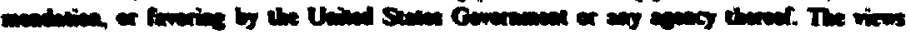

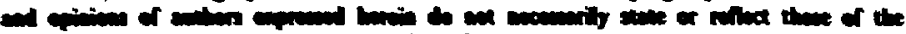

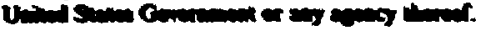




\section{Search for Right-Handed Currents \\ by Means of Muon Spin Rotation}

David Philip Stoker

Abstract

A muon spin rotation ( $\mu S R$ ) technique has been used to glace limits on right-handed weak currents in $\mu^{+}$decay. A beam of almost $100 \%$ polarized 'surface' muons obtained from the TRIUMF M13 beamline was stopped in essentially non-depolarizing $>99.99 \%$ pure metal foils. The $\mu^{+}$spins were precessed by $70-G$ or 110-G transverse flelds. Decay $e^{+}$ented within $225 \mathrm{mrad}$ of the beam dircction and with momenta above $4 \% \mathrm{MeV} / \mathrm{c}$ were momentum-analyzed to $0.2 \%$. Comparison of the $\mu$ SR signal amplitude with that expected for $(V-A)$ decay yields an endpolnt asymmetry $\varepsilon P_{\mu} \delta / \rho>0.9951$ with $90 \%$ confidence. In the context of manifest left-right symmetric models with massless neutrinos the results imply the $90 \%$ confidence limits $M\left(W_{2}\right)>381 \mathrm{GeV} / \mathrm{C}^{2}$ and $-0.057<6<0.044$, where $W_{2}$ is a predominantly right-handed gauge boson and $\zeta$ is the left-right mixing angle. Limits on $M\left(W_{2}\right)$ for $M\left(v_{\mu R}\right) \neq 0$ are also presented. The endpoint asymatry is used to deduce liaits on the $v_{\mu L}$ mass and helicity in $\pi^{+}$decay, non-(V-A) couplings in helicity projection form, and the ass scale of composite leptons. 
Table of Contents

Acknowledgments

Chapter 1. Introduction

Chapter 2. The Standard and Left-Right Symmetric Models . . . 4

2.1 The Standard Model: A Brief Review . . . . . . . . 4

2.2 The Left-Right Symmetric Model: An Introduction . . . 6

2.3 Neutrinos: Dirac or Majorana? . . . . . . . . . 8

2.4 The Low-Energy Hamiltonian . . . . . . . . . . 10

2.5 Limits on Right-Handed Currents . . . . . . . . . 12

Chapter 3. Muon Decay . . . . . . . . . . . . 14

3.1 Four-Fermion Contact Interaction . . . . . . . - 14

3.2 Mur Decay Asymmetry . . . . . . . . . . . . 15

3.3 Radiative Corrections - . . - . . . . . . . 18

3.4 Effects of Intermediate Vector Bosons . . . . . . . 21

3.5 Lorentz Structure . . . . . . . . . . . . . 23

Chapter 4. Muons in Matter - . - . . . - . - . . . . 26

4.1 Muon Deceleration and Theraalization . . . . . . . 26

4.2 Mwon Dejolarizetion in Scattering

From Unpolarized Electrons - . . . - . - . - 27

4.3 Spln-lattice Relaxation . . . . . . . . . . . 29

4.4 Spin-Spla Relaxation: uSR SIEal Dampinc . . . . . . 33 
Chapter 5. The Beamline and Apparatus . . . . . . . . . 39

5.1 The Beamline . . . . . . . . . . . . . . . 39

5.2 The Apparatus: An Overview . . . . . . . . . 42

5.2.1 The Solenoid . . . . . . . . . . . . . 45

5.2.2 The Spectrometer . . . . . . . . . . . 48

5.2.3 Proportional Chambers . . . . . . . . . 48

5.2.4 Drift Chambers . . . . . . . . . . . 49

5.2 .5 Scintillators . . . . . . . . . . 52

5.2.6 Stopping Targets . . . . . . . . . . 52

5.3 The Trigger . . . . . . . . . . . . 54

5.4 Data Acquisition . . . . . . . . . . . 58

Chapter 6. Event Reconstruction . . . . . . . . . 60

6.1 Wire Chamber Alignment . . . . . . . . . . 60

6.2 Muon Track Reconstruction . . . . . . . . . . 60

6.3 Positron Track Reconstruction _. . . . . . . . . 61

6.4 Extra Muons . . . . . . . . . . . . . 64

6.5 Momentum Reconstruction . . . . . . . . . 65

Chapter 7. Data Analysis . . . . . . . . . . . 69

7.1 Overview . . . . . . . . . . . . . . . 69

7.2 Positron Momentu Spectra . . . . . . . . . . 70

7.3 Positron Angular scceptance . . . . . . . . . . 72

7.4 Positron Momentia Acceptance . . . . . . . . 73

$\pi .5$ Monte Carlo Tests . . . . . . . . . . . 74

7.6 Data Fitting kesults . . . . . . . . . . . 76 
Chapter 8. Corrections and Systematics . . . . . . . . 86

8.1 Corrections . . . . . . . . . . . . . . 86

8.1.1 Muon Depolarization in Scattering with Electrons - 86

8.1.2 Coulomb Scattering . . . . . . . . . . 86

8.1.3 Extra Muons . . . . . . . . . . . . 88

8.1 .4 Cloud Muons . . . . . . . . . . . . 89

8.1.5 Longitudinal Field Component . . . . . . . . 90

8.1.6 Timing Errors . . . . . . . . . . . . 90

8.1 .7 Summary . . . . . . . . . . . . . . . 91

8.2 Systematic Errors . . . . . . . . . . . . . 93

8.2.1 Reconstruction of $\theta_{\mu}$ and $\theta_{\mathrm{e}}$. . . . . . . . 93

8.2.2 Momentum Calibration . . . . . . . . . . 94

8.2.3 Definition of $x=1 \quad$. . . . . . . . . . . 94

8.2.4 Energy-Loss Straggling . . . . . . . . . . 94

8.2.5 Muon Mean-Life . . . . . . . . . . . . 95

8.2.6 Summary . . . . . . . . . . . . . . . 95

Chapter 9. Results and Conclusions . . . . . . . . . 97

9.1 The Normalized Asymmetries . . . . . . . . . . 97

9.2 Right-Handed Current Limits with Massless Neutrinos . . 98

9.3 Limits on $M\left(W_{2}\right)$ with $M\left(v_{\mu R}\right) \neq 0 \quad$. . . . . . . . . 98

9.4 Litaits on $E P_{\mu} \delta / \rho$. . . . . . . . . . . . 101

9.5 Limits on $M\left(v_{\mu L}\right)$ and $v_{\mu L}$ Helicity in $*^{+}$Decay . . . . 103

9.6 Lorentz Structure Restrictions . . . . . . . . 104

9. $\pi$ Limits on Composite Leptons . . . . . . . . . . 104 
Appendix A. First-Order Optics of Solenoldal Fields . . • • . 107

Appendix B. Positron Energy-Loss Straggling - • . . • • • 110

Appendix C. Tables of Data Fit Results . . • • . • • • 112

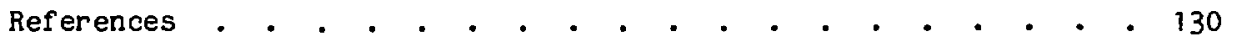




\section{Acknowledgments}

This thesis owes much to the efforts of the other collaboration members participating in TRIUMF experiments E185 and E247: Brian Balke, John Carr, George Gidal, Bruno Gobbi, Alex Jodid10, Chris Oram, Kirk Shinsky (deceased), Herb Steiner, Mark Strovink, and Bob Tripp. The technical assistanne of C. Covey, R. Fuzesy, F. Goozen, P.Harding, M. Morrison, and P. Robrish at L.B.L. and the support of the TRIUMF staff contributed to the success of the experiments.

I would particularly like to thank my advisor Mark Strovink, who having advised me initially that a thesis could be completed in $2+\varepsilon$ years, continued to provide patient support and advice as $\varepsilon$ grew to 2.7 .

I would also like to thank Marjorie Olmstead for helpful discussions of relevant aspects of sol1d state physics.

The assistance of Pat Bronnenberg in typing much of this thesis and other material is greatly appreciated.

The research reported here was supported in part by the U.S. Department, of Energy through Contracts No. DE-AC03-76SF00098 and AC02-ER02289. 
Chapter 1

Introduction

In the course of more than a decade of remarkable agreement with experiment the Glashow-Weinberg-Salam mode1 ${ }^{1-3}$ ), based on the gauge group SU(2) $\mathrm{LUU}(1)$, has become accepted as the 'standard model' of electroweak interactions. Despite its outstanding success the standard model does not explain the left-handed character of the charged surrent weak interactions such as $B$ and $\mu$ decay. Instead the left-handedness is built in a priori by allowing only the left-handed components of fermions to couple to the charged gauge bosons. Shortly before Weinberg and Salam unified the weak and electromagnetic interactions, Lipmanov4) asked

"... whether the nonconservation of parity in weak interactions is not a manifestation of a violated $\left(V_{ \pm A}\right)$ symmetry of these interactions, with $(V-A)$ dominance... It is possible that the coupling between the weak interaction currents is mediated by intermediate vector bosons. Then one can Imagine that there exist intermediate bosons of two kinds, $W^{(V-A)}$ and $W^{(V+A)}$, which nediate the $(V-A)$ and $(V+A)$ couplings, respectively. If the ans of the $W^{(V-A)}$ and $W^{(V+A)}$ were equal, there would be no experiental anifestation of parity non-conservation. However, the latter efrect appears if there is a was difference for the two Intermediate bocons. The effective current-current Lagrandian for the wak interactions... he the form (for $q^{2}\left(\mathrm{an}^{2}\right)$ :

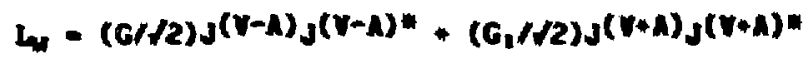


where $G / \sqrt{2}=4 \pi g^{2} / M^{2}(V-A), G_{1} / \sqrt{ } 2=4 \pi g^{2} / M^{2}(V+A)$ "

Lipmanov went on to show that the electron emission asymmetry in muon decay provided an estimate $G_{1} \leq 0.12 G$, and that the $\mu^{+}$from $\pi^{+}$ decay would be partially depolarized, with longitudinal polarization $P_{\mu}=1-2 G_{1}^{2} / G^{2}$

The more recent left-right syminetric theories 5,6$)$, in which the standard electroweak gauge group is extended to $S U(2)_{L} \times S U(2)_{R} \times U(1)$, embody the spirit of the Lipmanov formulation. Although completely left-right symmetric at the Lagranglan level these theories admit asymmetric solutions through spontaneous symmetry breaking which violate parity'). In particular, the Higgs mechanism can impart a larger mass to $W_{R}$ than to $W_{L}$, thereby suppressing the $r$ ght-handed currents at low $q^{2}$ while retainine parity conservation for $q^{2} \gg M^{2}\left(W_{R}\right)$.

This thesis presents the results of a search for deviations from the $(V-A)$ prediction for the $e^{+}$asymmetry in polarized $\mu^{+}$decay at rest by means of a muon spin rotation ( $\mu S R$ ) technique. The recent development") of 'surface' beams has provided muon beams with essentially the polarization intrinsic to pion decay at rest. Naturally, right-handed currents way contribute at each step of the $w+\mu+e$ decay chain thus enhancing the experiwental sensitivity.

The experinent was operated in two modes, each sensitive to right-handed ourrents but with different major sources of possible systematic error. In each asse the " bean was stopped in netal cargets. In metals, unitike many otmer atcrials, the pu are therwalized

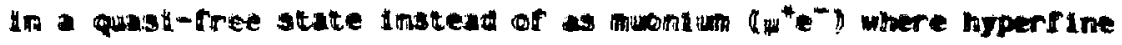

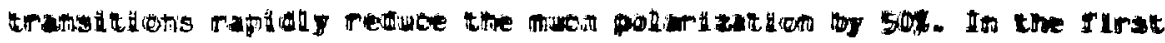


mode") the spins of the stopped $\mu^{+}$were held in a 1.1-T field which quenches muon depolarization in any residual muonium through the Paschen-Back effect. Measurement of the momentum spectrum endpoint decay rate opposite to the $\mu^{+} \mathrm{spin}$, which vanishes for a purely $(V-A)$ interaction, allows limits to be set on any right-handed current admixture. In the second mode, which provided the data presented here, the $\mu^{+}$spins were instead precessed by $70-G$ or $110-G$ fields transverse to the beam direction. The time variation of the $\mathrm{e}^{+}$emission rate near the beam direction as the $\mu^{+}$spins precess constitute the $\mu$ SR signal. Limits on right-handed currents are set by comparing the $\mu$ SR signal amplitude with that expected for a $(V-A)$ interaction.

The experiment was conceived in mid-1980 and most of the apparatus was constructed during 1981. The data presented in this thesis was accumulated during the three running periods of experiments E185 and E247 at the TRIUMF cyclotron during 1982-4. 
Chapter 2

The Standard and Left-Right Symmetric Models

2.1 The Standard Model: A Brief Review

The gauge group of the standard electroweak model is $S U(2)_{L} \times U(1)_{Y}$ with coupling constants $g$ and $g^{\prime}$ respectively. The leptons and quark weak eigenstates are assigned to left-handed $S U(2)$ doublets

$$
\left[\begin{array}{l}
v_{e} \\
e^{-}
\end{array}\right]_{L}\left[\begin{array}{l}
v_{L} \\
\mu^{-}
\end{array}\right]_{L, \ldots}\left[\begin{array}{l}
u \\
d^{\prime}
\end{array}\right]_{L,}\left[\begin{array}{l}
c \\
s^{\prime}
\end{array}\right]_{L, \ldots}
$$

and rlght-handed singlets.

The simplest Higgs assignment required to break down the symmetry to $U(1)_{\mathrm{em}}$, thereby guaranteeing the masslessness of the photon, is the scalar SU(2) Loublet

$$
\phi=\left[\begin{array}{l}
\phi_{0}^{+} \\
\phi^{0}
\end{array}\right]
$$

Minimizing the Higgs potential yields a non-zero vacuum expectation value solution

$$
+=\left[\begin{array}{l}
0 \\
v
\end{array}\right]
$$

which Iaparts asses to the $U$ and $Z$ bosons and the rermions. With the Weinberg angle $\theta_{w}$ defined by tang, $=g^{\prime} / g$ the gauge rields $\overrightarrow{H^{*}}=\left(W^{2}, w^{2}, W^{3}\right)$ and $B_{1}$ associated with $\operatorname{SU}(2)_{L}$, and $U(1)_{Y}$ respectively, become the pinysioal doson elenstates

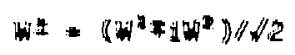$$
N^{2}=6^{2}+2 / 2
$$ 


$$
\begin{array}{ll}
Z=W^{3} \cos \theta_{W}-B \sin \theta_{W} & M_{Z}^{2}=\left(g^{2}+g \cdot 2\right) V^{2} / 2 \\
Y=W^{3} \sin \theta_{W}+B \cos \theta_{W} & M_{Y}=0
\end{array}
$$

Comparison of single $W$ exchange in the low-energy limit with the corresponding four-fermion contact interaction 81 ves $\mathrm{g}^{2} / 8 \mathrm{M}_{\mathrm{W}}{ }^{2}=\mathrm{G}_{\mathrm{F}} / \sqrt{2}$ where $\mathrm{G}_{\mathrm{F}}$ is the Fermi coupling constant. In addition, the form of the electromafnetic curren: allows the electronic charge $e=\sqrt{ }(4 \pi \alpha)$ to be related to $g$ and $g^{\prime}$ by $e=g \sin \theta_{W}=g^{\prime} \cos \theta_{W}$. Tinen to lowest order and ignoring radiative corrections the standard model predicts

$$
M_{W}=\frac{1}{\sin \theta_{W}}\left[\frac{\pi \alpha}{G_{F} \sqrt{2}}\right]^{1 / 2}=\frac{37.3}{\sin \theta_{W}} \mathrm{GeV}
$$

and

$$
M_{2}=\frac{M_{W}}{\cos \theta_{W}}-\frac{74.6}{\cos 2 \theta_{W}} \mathrm{GeV}
$$

Table (2.1) shows the experimental masses fiom the $U A-1^{10}$ ) and UA-2 ${ }^{12)}$ collaborations at CERN together with the standard model predictions of Marclano and $\left.\operatorname{sirlin}^{12}\right)$. The theoretical predictions use $\sin ^{2} \theta_{w}=0.217 \pm 0.014$ obtained from deep inelastic $v_{p}$ scartering and the e-D scattering asymetry after applying radiative corrections.

\begin{tabular}{cccc}
\hline & UA-1 & UA-2 & Standard Model \\
\hline$H_{1}$ (GeV) & $80.9 \pm 1.5 \pm 2.4$ & $31.0 \pm 2.5 \pm 1.3$ & $83.0+2.9$ \\
$H_{2}$ (GeV) & $95.6 \pm 1.5 \pm 2.9$ & $91.9 \pm 1.3 \pm 1.4$ & $93.8+2.4$ \\
\hline
\end{tabular}

Table (2.1)

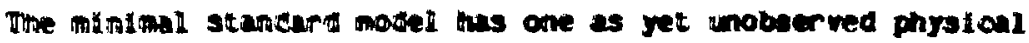


neutral scalar Higss with a mass $M_{H}$ not predicted by the theory. However, stability of the physical vacuim requires $\mathrm{H}_{\mathrm{H}}>7 \mathrm{GeV}$ and the weak interactions are predicted to become strong at high energies unless $M_{H}<1$ TeV.

\subsection{Left-Right Symmetric Mode 1 : An Introduction}

The gauge group of ieft-right symmetric models is SU(2) $)_{L} \times S U(2)_{R} \times U(1)_{B-L}$ with coupling constants $B_{L}, g_{R}$, and $B^{\prime}$ respectively. Only manifest left-right symmetric models, for which $\mathrm{B}_{\mathrm{L}}=\mathrm{g}_{\mathrm{R}}=\mathrm{g}$, are considered here. Compared to the standard model, the lert-right symmetric model requitres an extra set of gauge bosons and a more complex Higgs structure to produce the fermion and gauge boson masses. The lefi- and right-hancied fermion components are assigned to isospin doublets $\psi_{L}, R$ with the indicated quantum numbers $\left(T_{L}, T_{R}, B-L\right)$ :

$$
\begin{gathered}
{\left[\begin{array}{l}
v_{e}^{e} \\
e^{-}
\end{array}\right]_{L},\left[\begin{array}{l}
v_{\mu} \\
\mu^{-}
\end{array}\right]_{L}, \ldots\left[\begin{array}{l}
v_{e} \\
e^{-}
\end{array}\right]_{R},\left[\begin{array}{l}
v_{\mu} \\
\mu^{-}
\end{array}\right]_{R, \ldots}\left[\begin{array}{l}
u \\
d^{\prime}
\end{array}\right]_{L},\left[\begin{array}{l}
c \\
s^{\prime}
\end{array}\right]_{L}, \ldots\left[\begin{array}{l}
u \\
d^{\prime}
\end{array}\right]_{R,}\left[\begin{array}{l}
c \\
s^{\prime}
\end{array}\right]_{R, \ldots},} \\
(1 / 2,0,-1) \quad(0,1 / 2,-1) \quad(1 / 2,0,1 / 3) \quad(0,1 / 2,1 / 3)
\end{gathered}
$$

The generation of Dirac masses, $\alpha\left(\psi_{R} \psi_{L}+\psi_{L} \psi_{R}\right)$, for the fermions requires Yukawa couplings to Higgs multiplets with quantum numbers $\left(1 / 2,1 / 2^{*}, 0\right)$ since the ass terms in the Lagranglan must be Lorentz scalars. The required multiplets of complex scalar flelds are

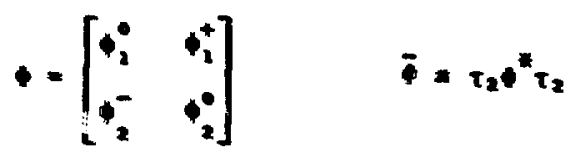


Additional Higgs multiplets are needed to complete the symetry breakdown to $\mathrm{U}(1)_{\mathrm{em}}$. The simplest choice is the doublets

$$
x_{L}=\left[\begin{array}{l}
x_{L}^{+} \\
x_{L}^{0}
\end{array}\right] \quad x_{R}=\left[\begin{array}{l}
x_{R}^{+} \\
x_{R}^{\circ}
\end{array}\right]
$$

with quantum numbers $(1 / 2,0,1)$ and $(0,1 / 2,1)$ respectively. Although the classical Higgs potential is symmetric under $X_{L^{\leftrightarrow}} \rightarrow X_{R}$, Senjanovic ${ }^{3}$ ) has shown that for a range of coefficients an asymmetric solution

$$
\left\langle X_{L}\right\rangle=0, \quad\left\langle X_{R^{\prime}}\right\rangle=\left[\begin{array}{l}
0 \\
v
\end{array}\right], \quad\langle \$\rangle=\left[\begin{array}{cc}
k & 0 \\
0 & k^{\prime}
\end{array}\right]
$$

emerges as the absolute minimum of the potential.

The gauge fields $\vec{w}_{L}, \vec{w}_{R}$, and $B$ associated with $S U(2)_{L}, \operatorname{SU}(2)_{R}$, and $\mathrm{U}(1)_{\mathrm{B}-\mathrm{L}}$ respectively, combine to form the mass eigenstates $W_{1} \pm, W_{2} \pm$, $z_{1}, z_{2}$ and $\gamma$. In general, the Higgs mechanism which gives masses to the gauge bosons also produces a left-right mixing. The physical charged bosons are

$$
\left[\begin{array}{l}
W_{1} \\
W_{2}
\end{array}\right]^{ \pm} \cdot\left[\begin{array}{cc}
\cos \zeta & \sin \zeta \\
-\sin \zeta & \cos \zeta
\end{array}\right]\left[\begin{array}{c}
W_{L} \\
W_{R}
\end{array}\right]^{ \pm}
$$

where $W_{L, R}^{ \pm}=\left(W_{L}^{2} R^{\mp i} W_{L, R}^{2}\right) / \sqrt{ } 2$ and $\tan 2 \zeta=-4 k k^{\prime} / V^{2}$. The experinental constraints that $\zeta$ is small and $M\left(W_{2}\right) \gg M\left(W_{i}\right)[\operatorname{section}(2.5)]$ inply $Y \gg K_{1} K^{\prime}$, and then

$$
\begin{aligned}
& H^{2}\left(H_{1}\right)=g^{2}\left(k^{2}+k^{12}\right) / 2 \\
& H^{2}\left(H_{2}\right)=g^{2}\left(v^{2}+k^{2}+k^{\prime 2}\right) / 2
\end{aligned}
$$

With 6, , the analog of the Hetnberg angle, defined by $\left.\sin ^{2} \theta^{\prime \prime}-g^{2} / g^{2}+g^{\prime 2}\right)$ the phyatol neutral bosons are 


$$
\begin{aligned}
& Y=\left(w_{L}^{3}+w_{R}^{3}\right) \sin \theta_{w^{\prime}}+B /\left(\cos 2 \theta_{w^{\prime}}\right) \\
& z_{2}=W_{L}^{\prime} \cos \theta_{W^{\prime}}{ }^{\prime}-W_{R}^{3} \sin \theta_{W}{ }^{\prime} \tan \theta_{W^{\prime}}{ }^{\prime}-B \tan \theta_{W} \cdot V\left(\cos 2 \theta_{W}{ }^{\prime}\right) \\
& z_{2}=W_{R}^{3} /\left(\cos 2 \theta_{W^{\prime}}{ }^{\prime}\right) / \cos \theta_{W^{\prime}}-B \tan \theta_{W^{\prime}}
\end{aligned}
$$

with masses

$$
\begin{aligned}
& M(\gamma)=0 \\
& M\left(z_{1}\right)=M\left(W_{1}\right) / \cos \theta_{W^{\prime}} \\
& M\left(z_{2}\right)=M\left(W_{2}\right) \cos \theta_{W^{\prime}}^{\prime} / V\left(\cos 2 \theta_{W^{\prime}}\right)
\end{aligned}
$$

In addition, for the above choice of Higgs multiplets, there remain six neutral and four charged physical Higgs scalars. In the model of Senjanovic $\left.{ }^{13}\right)$ one neutral Higgs has a mass $-\left(M\left(W_{2}\right)\right)$ and the rest have masses $-\left(M\left(W_{2}\right)\right)$.

In the limit $M\left(W_{2}\right) \rightarrow \infty$ the predictions of the left-right symmetric model are 1dentical to those of the standard model for both the charged and neutral currents. Also, in the limit $\zeta+0$ but with $M\left(W_{2}\right)$ finite both models make identical predictions for the parity violating neutral currents.

\subsection{Meutrinos: Dirac or Majorana?}

The $v_{L}$ of the standard electroweak model may be elther Dirac or Majorana particles. In the Dirac case $v_{L}$ and $v_{R}$ are different helicity states of the same particle, and $v_{R}$ is assigned to ar SU(2) singlet. However, for Majorana neutrinos $v_{L}$ and $v_{R}$ are different particles, and vif is absent from the standard nodel.

The situation is wore conplex in the left-right symetric model where, depending on the cholce of Hiecs structure, the neutrinoe ay 
acquire both Majorana and Dirac asses. As will be seen below this provides an explanation, first proposed by Gell-Mann, Ramond and Slansky ${ }^{24}$ ), for the smallness of the $v_{L}$ mass. It also has a najor impact on the observability or right-handed currents in low-energy processes [section (2.4)].

The Dirac and Majorana mass terms have the structures and $\left(T_{L}, T_{R}, B-L\right)$ quantum numbers:

$$
\text { Dirac: } \quad\left(\bar{v}_{R} v_{L}+\bar{v}_{L} v_{R}\right) \quad(1 / 2,1 / 2,0)
$$

Majorana: $\left(\bar{v}_{L}^{c} v_{L}+\bar{v}_{L} v_{L}^{c}\right)$ and $\left(\bar{v}_{R}^{c} v_{R}+\bar{v}_{R} v_{R}^{c}\right) \quad(1,0,-2)$ and $(0,1,-2)$

Only Dirac mass terms, through Yukawa couplings to the multiplet $\phi$, are possible for the Higgs assigrment of section (2.2).

Mohapatra and Senjanovi ${ }^{25}$ ) have proposed a model in which two Majorana neutrinos $v$ and $N$ are assigned to the lepton doublets

$$
\left[\begin{array}{c}
\nu_{\mathrm{eL}} \\
\mathrm{e}^{-}
\end{array}\right], \ldots \cdot\left[\begin{array}{c}
\mathrm{N}_{\mathrm{eR}} \\
\mathrm{e}^{-}
\end{array}\right], \ldots
$$

prior to spontaneous symmetry breaking, and the H1ggs multiplets $X_{L, R}$ are replaced by $\Delta_{L}(1,0,2)$ and $\Delta_{R}(0,1,2)$ which generate the additional Majorana nass terms. The new Higgs structure is sonewhat nore complicated with

$$
\Delta_{L, R}=\left[\begin{array}{cc}
\delta^{+} / \sqrt{2} & \delta^{++} \\
\delta^{*} & -\delta^{+} / / 2
\end{array}\right]_{\mathrm{L}, \mathrm{R}}
$$


$\left\langle\Delta_{R}{ }^{\circ}\right\rangle=v$, the Majorana mass tern for $v_{L}$ vanishes while that for $N_{R}$ is $-M\left(H_{2}\right)$. The off-diagonal Dirac mass teras $\left(-\mathrm{H}_{\ell}\right.$ for $\left.\ell=e, \mu, \tau\right)$ cause a slight left-right mixing so that the mass eigenstates $v_{1}$ and $v_{2}$ are

$$
\left[\begin{array}{l}
v_{1} \\
v_{2}
\end{array}\right]=\left[\begin{array}{ll}
\cos \delta & \sin \delta \\
-\sin \delta & \cos \delta
\end{array}\right]\left[\begin{array}{l}
v_{L} \\
N_{R}
\end{array}\right]
$$

with masses $\quad M\left(v_{\ell_{1}}\right)=M_{\ell}^{2} / M\left(W_{2}\right)$

$$
M\left(v_{\ell 2}\right)=M\left(W_{2}\right)
$$

and mixing angle $\delta \sim M_{Q} / M\left(H_{2}\right)$

Here the small mass of the predominantiy left-handed neutrino $v_{1}$ is clearly related to the suppression of the right-handed currerits through the asymmetrlo vacuum expectation values $\left\langle\Delta_{L}\right\rangle=0$ and $\left\langle\Delta_{R}{ }^{0}\right\rangle=v$.

\subsection{The Low Energy Hamiztonian}

In the case of Dirtiz: neutrinos $m\left(v_{R}\right)=m\left(v_{L}\right)$, which is known experimentally to be small. The effective low-energ. Hamiltonian for charged curirent processes is then

$$
\begin{aligned}
& H_{e r f}=\frac{g^{2}}{2 H^{2}\left(H_{3}\right)}\left\{J_{L} J_{L}^{\dagger}\left(\cos ^{2} \zeta+\operatorname{csin}^{2} \zeta\right)-J_{R} d_{R}^{\dagger}\left(\sin ^{2} \zeta+\cos ^{2} \zeta\right)\right. \\
& +\left(J_{L} J_{L}^{t}+J_{R} J_{L}^{t}\right)(t-\epsilon) \sin (\cos \zeta\}
\end{aligned}
$$

where the aiss-squared ratlo $\varepsilon=M^{2}\left(H_{1} ; / N^{2}\left(H_{2}\right)\right.$ and the left-right aixing angle $\zeta$ are sald. Retaining only the leating order terns

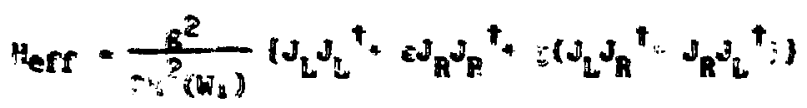


In the Majorana case described in section (2.3) the predominantiy right-handed $v_{2}$ is too massive to be produced in low-energy processes. The effective hamiltonian is now different or leptonic and semileptonic processes since $\mathbf{r l g h t - h a n d e d ~ c u r r e n t s ~ a r e ~ s u p p r e s s e d ~ b y ~ a ~}$ factor of sins at the leptonic vertices:

Semileptonic:

$$
\begin{aligned}
\mathrm{H}_{\mathrm{eff}}=\frac{\mathrm{g}^{2}}{2 \mathrm{M}^{2}\left(\mathrm{~W}_{1}\right)}\left\{\mathrm{J}_{\mathrm{L}} \mathrm{J}_{\mathrm{L}}^{\dagger}\right. & \cos \delta\left(\cos ^{2} \zeta+\varepsilon \sin ^{2} \zeta\right)-\mathrm{J}_{\mathrm{R}} \mathrm{J}_{\mathrm{R}}^{\dagger} \sin \delta\left(\sin ^{2} \zeta+\varepsilon \cos ^{2} \zeta\right) \\
& \left.+\left(\mathrm{J}_{\mathrm{R}} \mathrm{J}_{L}{ }^{\dagger} \cos \delta-\mathrm{J}_{\mathrm{L}} \mathrm{J}_{\mathrm{R}}{ }^{\dagger} \sin \delta\right)(1-\varepsilon) \sin \zeta \cos \zeta\right\}
\end{aligned}
$$

Leptonic:

$$
\begin{aligned}
H_{e f f}=\frac{g^{2}}{2 M^{2}\left(W_{1}\right)}\left(J_{L} J_{L}^{\dagger}\right. & \cos ^{2} \delta\left(\cos ^{2} \zeta+\varepsilon \sin ^{2} \zeta\right)+J_{R} J_{R}{ }^{\dagger} \sin ^{2} \delta\left(\sin ^{2} \zeta+\varepsilon \cos ^{2} \zeta\right) \\
& \left.-\left(J_{R} J_{L}{ }^{\dagger}+J_{L} J_{R}^{\dagger}\right)(1-\varepsilon) \sin \delta \cos \delta \sin \zeta \cos \zeta\right\}
\end{aligned}
$$

Then to leading order in $\varepsilon$ and $\zeta$, but neglecting terms in $\delta$ :

Sem1leptonic: $\quad H_{\text {eff }}=\frac{8^{2}}{2 M^{2}\left(w_{1}\right)}\left\{\left(J_{L} J_{L}^{\dagger}+\zeta J_{R} J_{R}^{\dagger}\right\}\right.$

where the right-handed current is purely hadronic.

Leptonic: $\quad H_{\text {erf }}=\frac{g^{2}}{2 M^{2}\left(w_{1}\right)} J_{L} J_{L}{ }^{\dagger}$

Thus if the $v_{2}$ are sufflclently massive, purely leptonic low-energy processes such as muon decay give no inforation on $c$ and $\zeta$ recardleas of $\mathrm{H}\left(\mathrm{H}_{2}\right)$, while sendleptonic processes still yleld inforation on 6 . The non-leptonlc lou-energy Hailtonlan is unchanged fro equation (2.1). 


\subsection{Linits on Right-Handed Currents}

The already exisitng experimental $90 \%$ confidence lidits on the mass-squared ratio $\varepsilon$ and the mixing angle $\zeta$ are aisplayed in Figure (2.1). The allowed regions are those which include $\varepsilon=\zeta=0$, 1.e. the $(V-A)$ limit. Only the linlts from the $y$ distributions in $v N$ and $\overline{v N}$ scattering (double 11nes, Ref, 16) are valld Irrespective of the $v_{R}$ mass. The other 11 mits assume massless or vary 11 ght $v_{\mathbb{R}}$. Muon decay contours are derived from decay-rate measurements opposite the $\mu^{+}$spin direction at the spectrum endpoint (bold curve, spin-held data from the present experiment, Ref. 9); the product of the asymmetry parameter and the $\mu^{+}$polarization, $E P_{\mu}$ (dotted curve, Ref. 17); and the Michel parameter $\rho$ (solid curve, Ref. 18). Nuclear $\beta$ decay contours are obtained from the Gamow-Teller $\beta$ polarization (dot-dashed curves, Ref. 19); the comparison of Gamow-Teller and Fermi $\beta$ polarizations (long-dashed curves, Ref. 20); and the "Ne asymetry $A(0)$ and $\mathrm{ft}$ ratio, with the assumption of conserved, vector current (short-dashed curves, Ref's. 21 and 22).

Additional model dependent limits, Independent of the $v_{R}$ mass but assuming the same left- and right-handed quark mixing angles, are set by scalleptonic decays $\left.{ }^{23}\right)[|\zeta|(1-\varepsilon)<0.005]$, current algebra analysis of non-leptonic $\Delta S=1$ weak decays $\left.{ }^{2+}\right)\left[|\zeta|(1-\varepsilon)<0.004\right.$, and $M\left(W_{2}\right)>300$ GeV if $6=0]$, and the $K_{L}-K_{S}$ mass difference ${ }^{2,26)}\left[M\left(W_{2}\right)>1.6\right.$ TeV]. Wh thout the quark wixing angle assuption the $K_{L}-K_{S}$ mass difference provides a Beneral lintt $\left.{ }^{*}\right) \mathrm{m}\left(\mathrm{H}_{2}\right)>300 \mathrm{GeV}$. 


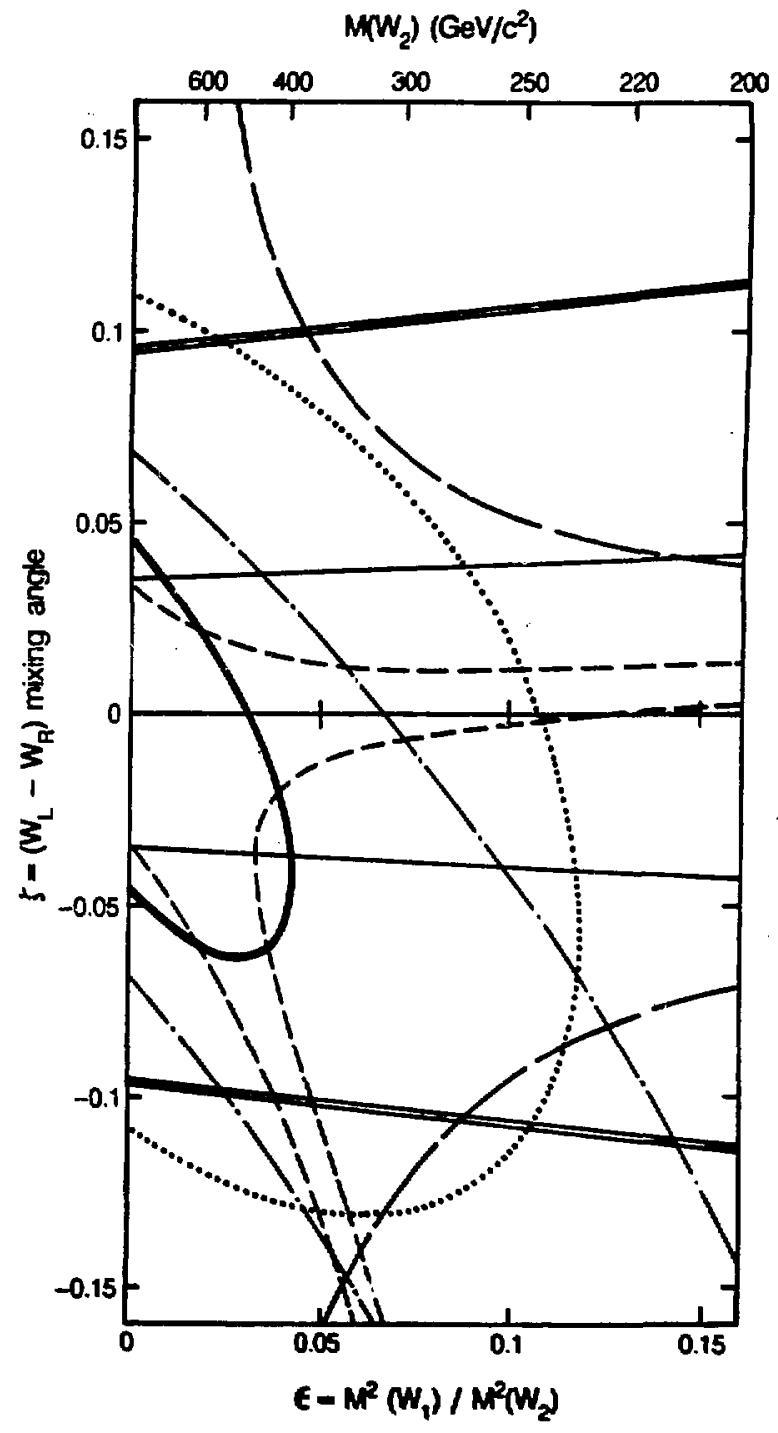

$x+205-1010$

FIGURE (2.1). Experimental 90\% confidence linits on the $w_{1}, 2$ mas-squared ratio $E$ and the left-richt mixing ancle 6 . The allowed regions are those which Include $c=t-0$. The sources of the linits are described in the text. 
Chapter 3

Mwon Decay

\subsection{Four-Fermion Contact Interaction}

The muon differential decay rate for an interaction mediated by a heavy vector boson, $W$, differs from that for the corresponding four-fermion contact interaction by terms $\left.{ }^{20}\right)$ of order $\left(m_{\mu} / M_{W}\right)^{2}$. These terms are $-10^{-6}$ for $\mathrm{M}_{\mathrm{W}}=80 \mathrm{GeV} / \mathrm{c}^{2}$ and are negligible at the present level of experimental precision. Consequently it is legitimate to treat muon decay as a contact interaction.

The $\mu^{+}$decay probability, integrated over $e^{+}$spin directions, for the most general four-fermion contact interaction with massless neutrinos and in the absence of radiative corrections $19^{22,30}$ )

$$
\begin{aligned}
\frac{d^{2} \Gamma}{d x d(\cos \theta)} \times & \left(x^{2}-x_{0}^{2}\right)^{1 / 2}\left\{9 x(1-x)+2 p\left(4 x^{2}-3 x-x_{0}^{2}\right)+9 \pi x_{0}(1-x)\right. \\
& \left.+\xi \cos \theta\left(x^{2}-x_{0}^{2}\right)^{1 / 2}\left[3(1-x)+2 \delta\left(4 x-3-m_{e} x_{0} / m_{\mu}\right)\right]\right\}
\end{aligned}
$$

Here $\theta$ is the angle between the $\mu^{+}$spin direction and the $e^{+}$momentum direction in the $\mu^{+}$rest frame, $x$ is the standard reduced energy $\operatorname{variable} x \cdot E_{e} / E_{e}(\max )$ where $E_{e}(\max )=\left(m_{\mu}{ }^{2}-m_{e}{ }^{2}\right) / 2 m_{\mu}=52.631 \mathrm{MeV}$, and $x_{0}=m_{e} / E_{e}(\max )$. The values of the muon decay parameters $\left.2,, 10\right) \rho_{0} n_{0}$ $E$, and $\delta$ depend on the relative strengths of the scalar, pseudoscalar, vector, axlal-vector and tensor interact Ions allowed by Lorentz Invariance. Table $(3.1)$ shows the $(V-1)$ and $(V+1)$ values of the decay parateters, together with thelr already existing experinental values"2). The values asuaed by the paraneters for more general foras or the interaction are discussed in section (3.5). 


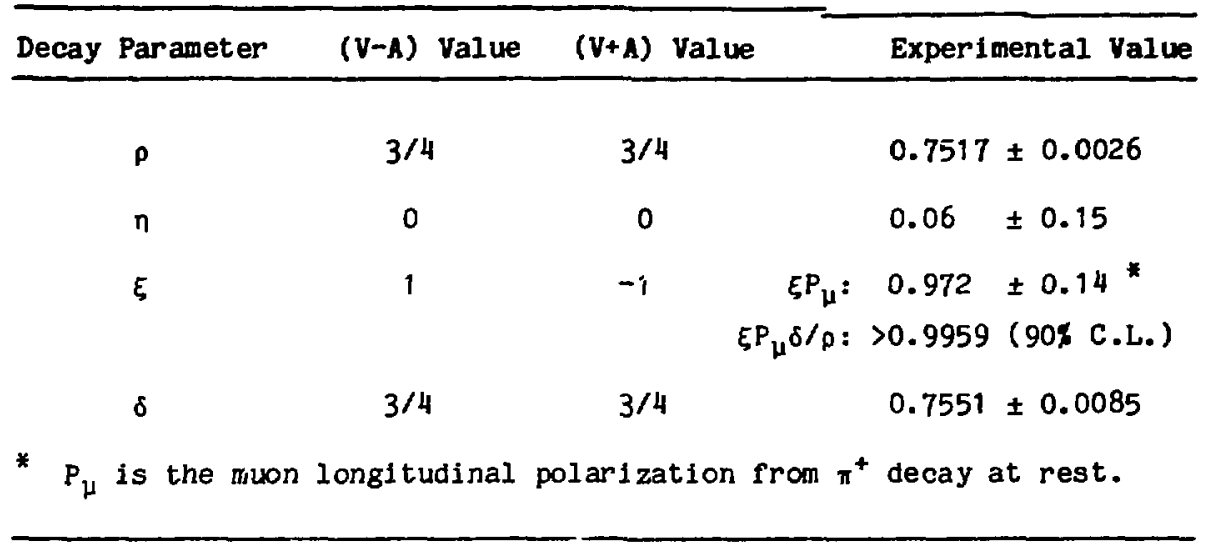

Table (3.1)

3.2 Muon Decay Asymmetry

In this section the muon decay asymmetry for arbitrary values of the decay parameters is compared to the (V-A) prediction ard is then related to the parameters $\varepsilon$ and $\zeta$ which characterize the left-right symmetric model.

Fran here on the term involving $n$ is assumed to be negliglble. In addition to $n$ being small experimentally [Table (3.1)], the term is suppressed by the factor $x_{0}=0.01$ and vanishes at the momentum spectru endpoint. To simplify the discussion surther the approximation $m_{e}=0$ is made teaporarily, yielding

$$
\left.\frac{d^{2} T}{d x d(\cos \theta)}=x^{2}(9: 1-x)+2 p(4 x-3)+E \cos \theta[3(1-x)+28(4 x-3)]\right\}
$$

If the ${ }^{*}$ spin direction is precessed in a manetic field the rate at which $e^{4}$ re colitted in a fixed direction becomes tive-dependent thrount the tiole-dependence of coso. The Inatantancous decay rate, 
normalized to the time-averaged $(\cos \theta=0)$ rate, is

$$
R[x, \theta(t)]=1+\frac{3(1-x)+2 \delta(4 x-3)}{9(1-x)+2 p(4 x-3)} \xi \cos \theta(t)
$$

The corresponding normalized rate for a purely ( $V-A)$ interaction $(\rho=\delta=3 / 4, \xi=1)$ is

$$
R[x, \theta(t)]_{(V-A)}=1+\frac{2 x-1}{3-2 x} \cos \theta(t)
$$

The maximum time variation of the rate, and hence the greatest experimental sensitivity to the degree of parity violation, is attained at $x=1$ and for maximal variations of $\cos \theta(t)$. The spin-precessing magnetic.field should therefore be perpendlcular to the $\mu^{+}$spin direction. The decays of most interest are those in which the $\mathrm{e}^{+}$is emitted with $x$ near 1 in a direction close to the $\mu^{+}$spin precession plane.

The amplitude of the resulting uSR signal, normalized to that expected for pure V-A muon decay, is

$$
A(x)=\frac{R[x, \theta(t)]-1}{R[x, \theta(t)](V-A)-1}
$$

and with the definitions $\bar{x}=1-x, \bar{\delta}-1-4 \delta / 3$ and $\bar{p}=1-4 \rho / 3$

$$
A(\bar{x})=(E \delta / p)(1+2 \bar{x}[\bar{\delta} /(1-2 \bar{x})-3 \bar{p} /(1+2 \bar{x})]]
$$

In the $\left(Y_{ \pm} A\right)$ lialts $A(\bar{x})=* 1$. For sall $\bar{x}$ the $(V-A)$ values of $\rho$ and $\delta$ may be inserted into equation (3.2) provided $E$ is then replaced by $A(\tilde{x})$. in aditional modification to equation (3.2) is required because the Incoming $w^{*}$ spin direction cannot be observed experimentally. However, th the $(V-A)$ IImit with wasless reutrlinos anguhar monentum 
conservation requires the $\mu^{+}$from $\pi^{+}$decay at rest to be enitted with their spin and momentum directions anti-parallel. Deviations from this limit can only reduce the longitudinal polarization $P_{\mu}$. With $\theta$ redefined to be the angle between the observed $\mu^{+}$and $e^{+}$momenta, equation (3.2) becomes

$$
\frac{d^{2} \Gamma}{d x d(\cos \theta)} \propto x^{2}\left(3-2 x+P_{\mu} A(\tilde{x}) \cos \theta(1-2 x)\right)
$$

The quantity $P_{\mu} A(\tilde{x})$ is the amplitude of the $\mu S R$ signal normalized to that expected for $(V-A)$ decay of $\mu^{+}$with $P_{\mu}=1$. In the context of left-right symmetric theories values of $P_{\mu} A(\bar{x})<1$ imply the existence of right-handed currents or $m\left(\nu_{\mu}\right)>0$.

The remainder of this section is devoted to relating $P_{\mu} A(\tilde{x})$ to the mass-squared ratio $E=M^{2}\left(W_{1}\right) / M^{2}\left(W_{2}\right)$ and mixing angle $\zeta$ of the left-right symmetric model. Following Beg et al. ${ }^{\prime 2}$, the effective low-energy Lagrangian may be written as

$$
L_{e f f}=-(G / 2)\left[v_{\lambda}^{\dagger} v^{\lambda}+n_{a a_{\lambda}} A^{\dagger} A^{\cdot} \cdot{ }^{\prime}+\eta_{a v}\left(v_{\lambda}{ }^{\dagger} A^{\lambda}+A_{\lambda}{ }^{\dagger} v^{\lambda}\right)\right]
$$

where $V$ and $A$ are the vector and axial-vector parts of $J_{L}$ and $J_{R}$. With $M_{1}=M\left(W_{2}\right)$, and $M_{2}=M\left(W_{2}\right)$ :

$$
\begin{aligned}
G / \sqrt{ } 2 & =\left(e^{2} / 8 M_{1}^{2}\right)(\cos \zeta-\sin \zeta)^{2}+\left(g^{2} / 8 M_{2}^{2}\right)(\cos \zeta+\sin \zeta)^{2} \\
\eta_{a a} & =\left(x^{2} M_{2}^{2}+M_{1}^{2}\right) /\left(x^{2} M_{2}^{2}+M_{2}^{2}\right) \\
\eta_{a v} & =-K\left(M_{2}^{2}-M_{1}^{2}\right) /\left(x^{2} M_{1}^{2}+M_{2}^{2}\right) \\
K & =(1+\tan \zeta) /(1-\tan \zeta)
\end{aligned}
$$

The muon decay parameters are dow:

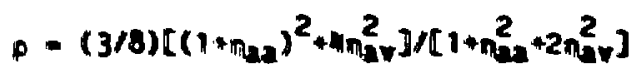




$$
\begin{aligned}
& n=0 \\
& \xi=-2 n_{a v}\left(1+n_{a a}\right) /\left[1+n_{a a}^{2}+2 n_{a v}^{2}\right] \\
& \delta=3 / 4 .
\end{aligned}
$$

and to leading order

$$
\begin{aligned}
\xi \delta / \rho & =1-2 \epsilon^{2} \\
\tilde{\rho} & =2 \zeta^{2}
\end{aligned}
$$

The $\mu^{+}$from $\pi^{+}$decay at rest have the polarization characteristic of Gamow-Teller $\beta$ decay:

$$
P_{\mu}=-2\left(n_{a a} / n_{a v}\right) /\left[1+\left(n_{a a} / n_{a v}\right)^{2}\right]=1-2(\varepsilon+\zeta)^{2}
$$

Equation (3.3) may now be rewritten in terms of $\varepsilon$ and $\zeta$ :

$$
P_{\mu} A(\tilde{x})-1-2\left\{2 \varepsilon^{2}+2 \varepsilon \zeta+\zeta^{2}[1+6 \tilde{x} /(1+2 \tilde{x})]\right\}
$$

Each value of $P_{\mu} A(\bar{x})<1$ is assoclated with an ellipticaj contour in the real $\varepsilon-\zeta$ plane. Thus measurement of $P_{\mu} A(\tilde{x})$ constrains both $\varepsilon$ and $\zeta$.

\section{3 Radiative Corrections}

Radiat 1 ve corrections to muon decay have been evaluated in detail only to order $a$. The first-order corrections are given by the virtual photon diagrams in Figure $(3.1)(a)-(c)$ and the inner bremsstrahlung diazrams (d) and (e) corresponding to the radiative decay $\mu \rightarrow$ evĩr. Fischer and Scheck') have calculated the radiative corrections for $(y-A)$ decay in the case unere the electron polarization 1 s not sumed over. The corrections independent or electron spin direction are unchanged if the (V-A) interaction is replaced by a more general vector and axlil-vector imteraction in charge retention form. Florescu and 

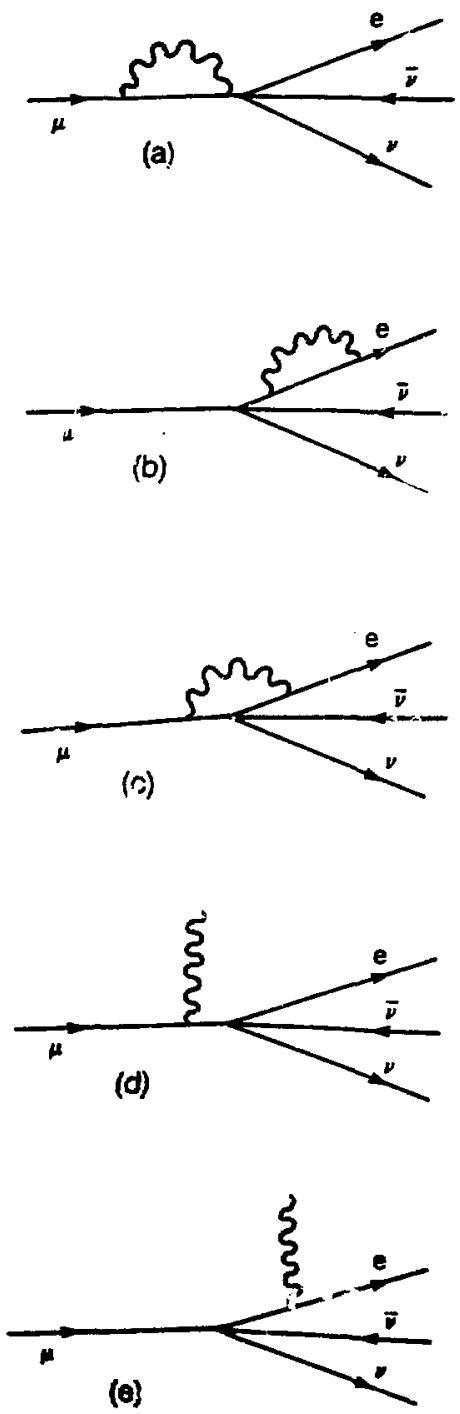

$x 02053-10: 41$

FIOURE (3.1). First-xcer radiative corrections to auon decay rroa virtul photon diaprang $(v)-(c)$, and laternal breasstrahlung diagrans (a) and (e). 
Kanei ${ }^{\text {"4) }}$ have calculated radiative corrections for a general Ferni Interaction. Including order a radiative corrections for $(V-A)$ decay and finite electron mass equation (3.4) becomes $2,, 10)$

$$
\begin{aligned}
\frac{d^{2} \Gamma}{d x d(\cos \theta)} \times\left(1-x_{0}^{2} / x^{2}\right)^{1 / 2}\left\{\left[x^{2}\left(3-2 x-x_{0}^{2} / x\right)+f_{c}(x)\right]\right. \\
\left.+P_{\mu} \wedge(\tilde{x})\left(1-x_{0}^{2} / x^{2}\right)^{1 / 2}\left[x^{2}\left(1-2 x+m_{e} x_{0} / m_{\mu}\right)+f_{\theta}(x)\right] \cos \theta\right\}
\end{aligned}
$$

where

$$
\begin{aligned}
f_{c}(x)= & (\alpha / 2 \pi) x^{2}\left\{2\left(3-2 x-x_{0}^{2} / x\right) R(x)-3 \ln x\right. \\
& \left.+\left[(1-x) / 3 x^{2}\right]\left[\left(5+17 x-34 x^{2}\right) \ln \left(m_{\mu} x / m_{e}\right)+2 x(17 x-11)\right]\right\} \\
f_{\theta}(x)= & (\alpha / 2 \pi) x^{2}\left\{2\left(1-2 x+m_{e} x_{0} / m_{\mu}\right) R(x)-\ln x\right. \\
& -\left[(1-x) / 3 x^{2}\right]\left[\left(1+x+34 x^{2}\right) \ln \left(m_{\mu} x / m_{e}\right) * 3-7 x-32 x^{2} \cdot 4(1-\cdot \ln (1-x) / x]\right\} \\
R(x)= & {\left[\ln \left(m_{\mu} x / m_{e}\right)-1\right]\left[2 \ln \left(x^{-1}-1\right)+3 / 2\right]+\ln (1-x)\left[\ln x+1-x^{-1}\right] } \\
& -\ln x+2 L_{2}(x)-\pi^{2} / 3-1 / 2
\end{aligned}
$$

and the Spence function $L_{2}(x)=-\int_{0}^{x} t^{-1} \ln (1-t) d t$.

It should be noted that $R(x)$, and hence $f_{c}(x)$ and $f_{\theta}(x)$, diverge logarithmically a: $x+1$. Qualitatively, the infrared divergences in the virtual photon dilagrams are no ionger compensated by those of the inner bremsstrahlung diagrams since the phase space for radiative decay vanishes as $x+1$. These divergences may be eliminaicad by including multiple soft-photon enission. The main effect near $x=1$ is to replace $1+(2 \alpha / x)\left[\ln \left(m_{\mu} / x_{e}\right)-1\right] \ln (1-x)$ in $R(x)[\text { equation }(3.9)]^{\prime}$ by" $)$

$$
\exp \left[(2 \alpha / x)\left[\ln \left(m_{\mu} / m_{e}\right)-1\right] \ln (1-x)-(1-x)(2 \alpha / x)\left[\ln \left(m_{\mu} / m_{e}\right)-1\right]\right.
$$

which vanishes as $x+1$ instead of diverging. It rollows that an appr:-inate correction of order $a^{2}$ any be made near $x=1$ by replacing 
$R(x)$ uth

$$
R_{2}(x)=R(x)+(2 \alpha / \pi)\left[\ln (1-x)\left[\ln \left(\mathbb{H}_{\mu} / \pi_{e}\right)-1\right]\right\}^{2}
$$

although, of course, $R_{2}(x)$ still diverges as $x+1$.

The data analysis uses equation (3.6) together with the radiative corrections or equations (3.7) through (3.10) to represent the $\mu^{+}$ differential decay rate. Figure (3.2) stwws the resulting $e^{+}$momentum spectra parallel and anti-parallel to the $\mu^{+}$spin. The radiative corrections are clearly not negligible.

3. 4 Effects of Intermediate Vector Bosons

As noted in section (3.1) the $\mu^{+}$differential decay rates for the $(V-A)$ contact interaction and the $W_{L}$-mediated interaction differ by terms of order $\left(m_{\mu} / M_{w}\right)^{2}$. The effect may be approximated by modifying the decay parameters as ${ }^{2 \bullet}$

and the decay rate as

$$
\begin{aligned}
\xi_{w} & =1+3 m_{\mu}^{2} / 5 M_{w}^{2} \\
\rho_{w} & =3 / 4+m_{\mu}^{2} / 3 M_{w}^{2} \\
\tau_{w}^{-1} & =\tau^{-1}\left(1+3 m_{\mu}^{2} / 5 M_{w}{ }^{2}\right)
\end{aligned}
$$

In addition the order a radiative corrections contain extra terms $\left.{ }^{3}\right)$ of order $\alpha\left(m_{\mu} / K_{\psi}\right)^{2}$. These effects are all negligible in the. present experinent. 


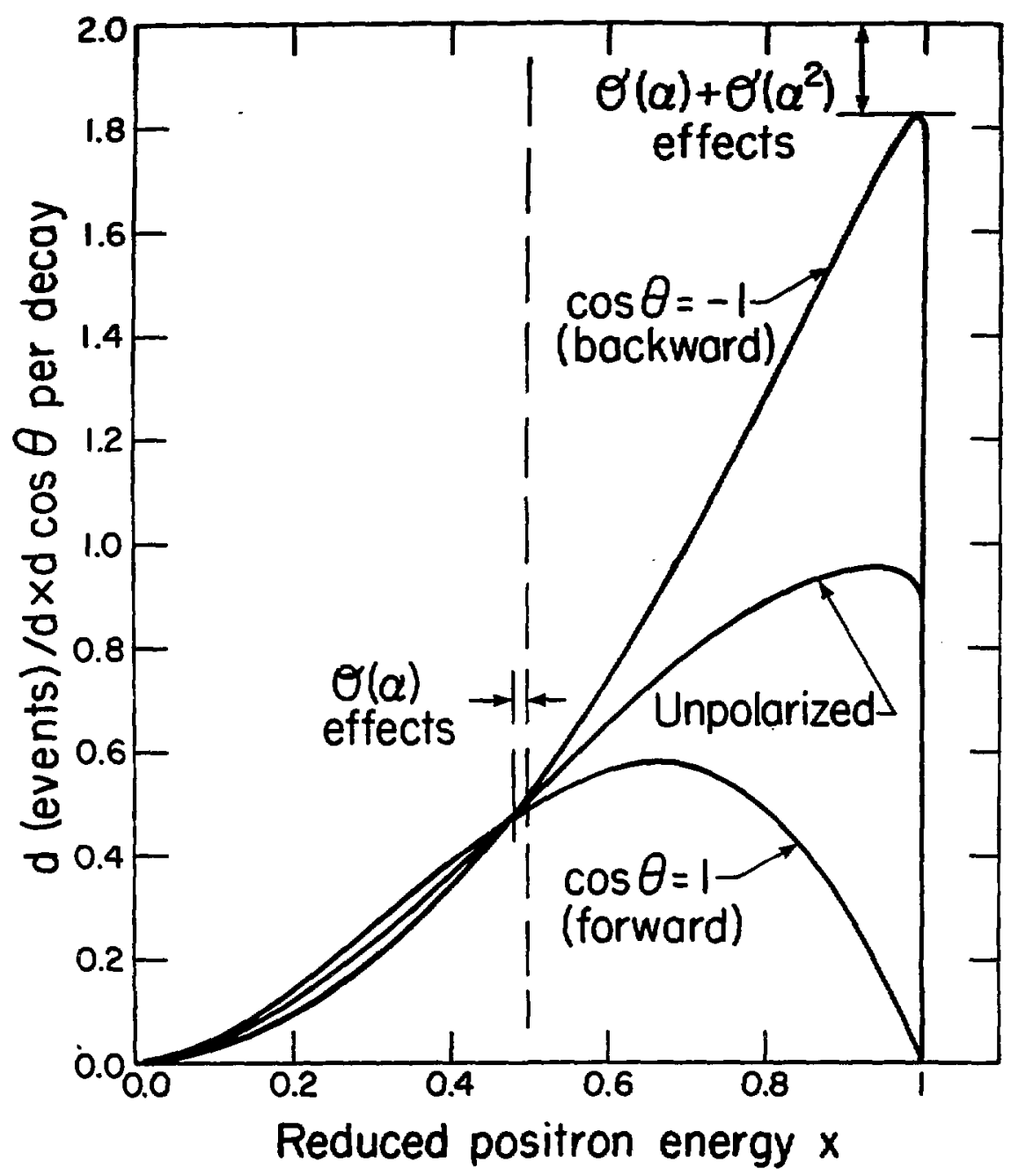

X8L8II-2103

FIGURE (3.2). The $(V-\Lambda) \mu^{*}$ difrerential decay rate parallel (backward) and ant1-parallel (forward) to the $p^{*}$ spin direction, and for unpolarized $\nu^{+}$. The effects of radiative corrections are also Indicated. 


\subsection{Lorentz Structure}

Mursula and Scheck ${ }^{26)}$ have recently obtained linits on non-( $(V-A)$ couplings using a hellcity projection form of the muon decay flavor retention contact interaction:

$$
\begin{aligned}
& H=\left(G_{0} / \sqrt{2}\right)\left(h_{12}(s+p) e v_{e}{ }^{(s+p)} v_{\mu \mu}+h_{12}(s+p)(s-p)+h_{21}(s-p)(s+p)\right. \\
& +h_{22}(s-p)(s-p)+g_{11}\left(v^{\alpha+\alpha} \alpha\right)\left(v_{\alpha}+a_{\alpha}\right)+g_{12}\left(v^{\alpha+a^{\alpha}}\right)\left(v_{\alpha}-a_{\alpha}\right) \\
& +g_{21}\left(v^{\alpha}-a^{\alpha}\right)\left(v_{\alpha}+a_{\alpha}\right)+g_{22}\left(v^{\alpha} a^{\alpha}\right)\left(v_{\alpha}-a_{\alpha}\right) \\
& \left.+f_{12}\left(t \alpha \beta+t^{\prime} \alpha \beta\right)\left(t_{\alpha \beta^{+}+t^{\prime} \alpha \beta}\right)+f_{22}\left(t^{\alpha \beta-t} \cdot \alpha \beta\right)\left(t_{\alpha \beta}-t^{\prime} \alpha \beta\right)+\text { h.c. }\right\}
\end{aligned}
$$

where $s_{1 k}=\bar{\psi}_{1} 1 \psi_{k}, p_{1 k}=\bar{\psi}_{1} \gamma_{s} \psi_{k}, v_{1 k}^{\alpha}=\bar{\psi}_{1} \gamma^{\alpha} \psi_{k}, a_{1 k}^{\alpha}=\bar{\psi}_{1} \gamma^{\alpha} \gamma_{s} \psi_{k}$, $t_{1 k}^{\alpha \beta}=\bar{\psi}_{1}\left(0^{\alpha \beta} / \sqrt{ } 2\right) \psi_{k}, t_{1 k}^{\alpha \beta}=\bar{\psi}_{1}\left(0^{\alpha \beta} \gamma_{s} / \sqrt{ } 2\right) \psi_{k}$ and the particle indices are as indicated in the $h_{11}$ term.

The pure (V-A) interaction is very simple in this form: only $g_{22}=0$. The combinations of covariants in each term project onto states of definite helicity in the limit of massless particles, and eliminate interference terms except between (scalar \pm pseudoscalar) and tensor interactions.

The deviations of the muon decay parameters from their ( $V-A)$ values are

$$
\begin{aligned}
& p-3 / 4=-(12 / A)\left\{\left|g_{12}\right|^{2}+\left|g_{21}\right|^{2}+2\left|f_{11}\right|^{2}+2\left|f_{22}\right|^{2}+\operatorname{Re}\left(h_{11} f_{11} * h_{22} f_{22}^{*}\right)\right\} \\
& \delta-3 / 4=(36 / A E)\left\{\left|g_{12}\right|^{2}-\left|g_{21}\right|^{2}-2\left|f_{12}\right|^{2}+2\left|f_{22}\right|^{2}-\operatorname{Re}\left(h_{11} r_{.1}^{*}-h_{22} f_{22}^{*}\right)\right\} \\
& E-1=-(8 / A)\left(4\left(\left|B_{11}\right|^{2}+2\left|g_{12}\right|^{2}-\left|g_{21}\right|^{2}\right)+\left|h_{11}\right|^{2}+\left|h_{21}\right|^{2}-4\left|f_{12}\right|^{2}+16\left|f_{22}\right|^{2}\right. \\
& \left.-8 R_{0}\left(h_{12} f_{22}^{*}-h_{22} f_{22}^{*}\right)\right] \\
& n=(8 / A) R e\left[g_{21}\left(h_{22} *+6 f_{22}^{*}\right)+g_{22}\left(h_{12} *+6 f_{22}^{*}\right)+g_{22} h_{21} *+g_{12} h_{12} *\right]
\end{aligned}
$$

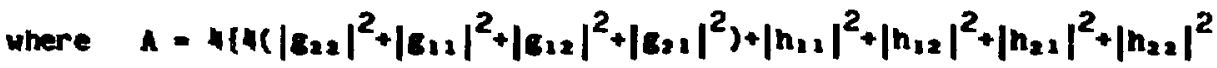

$$
\begin{aligned}
& \left.+12\left(\left|f_{21}\right|^{2}+\left|f_{22}\right|^{2}\right)\right]
\end{aligned}
$$


The couplings are related to equation (3.3) by equation (3.12):

$A(0)=\xi \delta / p=1-\frac{8\left|g_{11}\right|^{2}+2\left|h_{21}\right|^{2}+2\left|h_{11}-2 f_{11}\right|^{2}}{4\left(\left|g_{12}\right|^{2}+\left|g_{22}\right|^{2}\right)+\left|h_{12}\right|^{2}+\left|h_{21}\right|^{2}+\left|h_{11}-2 f_{12}\right|^{2}+\left|h_{22}-2 f_{22}\right|^{2}}$

Measurement of $P_{\mu} A(0) \leq A(0)$ therefore allows limits to be set on the couplings $g_{11}, h_{11}, h_{21}$, and $f_{12}$. Limits from the present experiment are presented in section (9.6).

Several constraints are imposed on the couplings if it is assumed that (1) the charged weak interactions are mediated by heavy bosons with spin 0, 1, or 2, (ii) the vector and tensor boson couplings are e- $\mu$ universal, and (iii) the scalar boson coupling may instead be proportional to the lepton mass (weak universality):

$$
\begin{aligned}
& h_{12}, h_{21} \text { real, positive semi-definite } \\
& h_{22}=h_{11} * \text { with }\left|h_{11}\right|^{2}=h_{12} h_{21} \\
& g_{11}, g_{22} \text { real, positive semi-definite } \\
& g_{21}=g_{12} * \text { with }\left|g_{12}\right|^{2}=g_{11} g_{22} \\
& f_{22}=f_{12}
\end{aligned}
$$

Limits on $g_{12}, h_{13}, h_{21}$, and $f_{11}$ therefore constrain other couplings. It should also be noted that any deviation of $\delta$ from $3 / 4$ would indicate a violation of $e^{-\mu}$ universality.

Two speclal cases are of Interest:

1) In the standard electroweak wodel where the charged weak Interaction 15 mediated by a single heavy vector boson $W^{ \pm}$which couples uni versally

$$
A(0)=1-2\left|8_{11}\right|^{2} /\left(\left|8_{12}\right|^{2}+\left|8_{22}\right|^{2}\right)
$$

$$
P_{1}=\left(8_{22}-8_{12}\right) /\left(8_{22}+E_{11}\right)
$$


so that

$$
P_{y} R(0)=1-2 B_{21} / B_{22}
$$

2) In the context of the left-right symetric model $\mathrm{G}_{11}$ and $\mathrm{G}_{12}$ provide weasures of $\varepsilon$ and $\zeta$. 
Chapter 4

\section{Muons in Matter}

4. 1 Muon Deceleration and Thermalization

The deceleration and thermalization of $\mu^{+}$in matter has been reviewed by Brewer et al.37) The main energy-loss processes depend on the $\mu^{+}$energy. For kinetic energies $E>2-3$ keV the energy loss is by scattering with electrons. The $\mu^{+}$beam is partially depolarized through spin exchange with the unpolarized electrons of the medium ${ }^{3}$ ). The calculation in section (4.2) shows the depolarization to be $7 \times 10^{-4}$ for surface muons. In addition, multiple Coulomb scattering from nuclei, which is non-relativistically spin conserving, misaligns the $\mu^{+}$spin and momentum directions. At $E=2-3 \mathrm{keV}$ the $\mu^{+}$velocity is comparable to that of the valence electrons of the medium. The $\mu^{+}$then begin to capture and lose electrons rapidly, forming a succession of short-lived muonium $\left(\mu^{+} e^{-}\right)$states. Again energy is lost in collisions with electrons. Below E=200 eV stable muonium is formed, and the energy loss is due to collisions of muonium with atoms and molecules. The time spent by the decelerating $\mu^{+}$in muonium states is too short for the hyperfine transitions to cause any appreciable depolarization.

In many non-metals the $\mu^{+}$are thermalized as muonium. In others, muonium with E=1-20 eV participates in 'hot atod' reactions where the $\mu^{+}$becose incorporated into molecules. The stopping targets in the present work were elther metals or liquid hellua. The $\mu^{+}$are thermalized in metals in a quasi-free state because the high conduction electron concentration effectively screens the "from Interactions

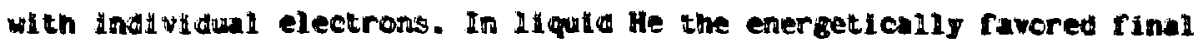


state 1 s the nolecular Ion He, with binding energies's) of 1.9 eV for the ground state and 1.2 ev for the first vibrational state. Muoniun is strongly disfavored in the final state due to the large difference between the ionization potentials of helium $(24.6 \mathrm{eV})$ and muonium $(13.5 \mathrm{eV})$. However, if any muons are thermalized as muonilu they may survive in this form for a considerable time because of the improbability of encountering a $\mathrm{He}^{+}$ion with which to recombine as $\mathrm{He}^{+}+\mu^{+} e^{-}+\mathrm{He}^{+}$.

4.2 Muon Depolarization in Scattering from Unpolarized Electrons

Ford and Mullin ${ }^{3}$ ) have shown that when non-relativistic $\mu^{+}$, with velocity $B$ in the laboratory frame, scatter with unpolarized $e^{-}$through a center of mass angle $\theta$ the probability that the final $\mu^{+}$spin direction is parallel $(\varepsilon=1)$ or anti-parallel $(\varepsilon=-1)$ to the initial spin direction 1s:

$$
Q(\varepsilon, \theta)=\frac{1+\varepsilon}{2}-\varepsilon \frac{m^{2}}{\mu^{2}} \beta^{4}\left[\sin ^{2}(\theta / 2)-\sin ^{4}(\theta / 2)+\sin ^{6}(\theta / 2)\right]
$$

where $m=m_{e}$ and $\mu=m_{\mu}$.

If the muons are initially fully polarized the final polarization after one scatter through $\theta$ is

$$
P_{\mu}=1-2 \frac{m^{2}}{\mu^{2}} B^{4}\left[\sin ^{2}(\theta / 2)-\sin ^{4}(\theta / 2)+\sin ^{6}(\theta / 2)\right]
$$

The corresponding fractional energy loss is

$$
u=\frac{n}{u} \sin ^{2}(0 / 2)
$$


With $\Delta P_{\mu}=1-P_{\mu}$ the 'depolarizing power' of a given fractional energy loss is

$$
\frac{\Delta P_{\mu}}{w}=2 \frac{m}{\mu} \beta^{2}\left[1-\sin ^{2}(\theta / 2)+\sin ^{4}(\theta / 2)\right]
$$

and

$$
\frac{d}{d\left[\sin ^{2}(\theta / 2)\right]} \frac{\Delta P_{\mu}}{W}=2 \frac{m}{\mu} \beta^{2}\left[-1+2 \sin ^{2}(\theta / 2)\right]
$$

The depolarization per unit energy loss is maximized for $\theta \rightarrow 0$ and $\pi$, and is reduced by $25 \%$ at the $\theta=\pi / 2$ minimum. In the non-relativistic limit the scattering cross section $0 \sim \operatorname{cosec}^{4}(\theta / 2)$. Then considering only small angle scattering the polarization after one scatter is

$$
P_{\mu}=1-2 \frac{m^{2}}{\mu^{2}} \beta^{4} \sin ^{2}(\theta / 2)
$$

With corresponding energy-loss

$$
d E=-E W=-\mu(\gamma-1) W=-m(\gamma-1) B^{2} \sin ^{2}(\theta / 2)
$$

The number of such scacters resulting in an energy loss $\delta \mathrm{E}$ such that $d E \ll \delta E \ll E$ is

$$
N=\frac{\delta E}{d E}=\frac{\delta E}{m(\gamma-1) \beta^{2} \sin ^{2}(\theta / 2)}
$$

and the polarization is then

$$
\begin{aligned}
P_{\mu}(\delta E) & =\left[1-2 \frac{m^{2}}{\mu^{2}} \theta^{4} \sin ^{2}(\theta / 2)\right]^{N} \\
& =1-2 \frac{m^{2}}{\mu^{2}} \frac{\beta^{2}}{\gamma-1} \delta E \\
& =1-2 \frac{m}{\psi^{2}} \frac{\gamma+1}{\gamma^{2}} \delta E
\end{aligned}
$$


The depolarization of non-relativistic $(\gamma=1)$ muons is therefore almost independent of their energy and proportional to their energy loss. Surface muons initlally have $E=4.1 \mathrm{MeV}$ and $\gamma=1.04$. Using $\Delta E=4$. 1Mev and $\gamma=1.02$ the depolarization when the $\mu^{+}$are (almost) brought to rest is

$$
1-P_{\mu}=2 \frac{m}{\mu^{2}} \frac{\gamma+1}{\gamma^{2}} \Delta E=7.3 \times 10^{-4}
$$

\subsection{Spin-Lattice Relaxation}

In order to obtain the most precise value of the measured muon mean-life $\tau_{\mu}$ to use in fitting the $\mu S R$ data one would like to include information from the spin-held mode of the experiment. However, muon spin-lattice relaxation in the spin-held mode conspires with parity violation to change the measured $\tau_{\mu}$ from 1 ts true value.

It should first be pointed out that while the 1.1-T spin-holding field is sufficlent to quench $\mu^{+}$depolarization in muonium, it cannot 'hold' the spins of quasi-free muons in the metal targets. The energy difference between states where the muon spin is parallel and ant1-parallel to the 1.1-T field is only $\Delta E=6.2 \times 10^{-7} \mathrm{eV}$, whereas the room temperature thermal energy is $\mathrm{kT}=2.6 \times 10^{-2} \mathrm{eV}$. Relaxation of the muon spins toward the equilibeiun situation, where the numbers of spins ant1-parallel and parallel to the applied field are almost equal, requires the presence of oscillating aagnetic flelds with frequency $\omega=9 \times 10^{8} \mathrm{~s}^{-1}$. Such fields are provided by the nuclear magnetic dipole monents and the lattice vibrations associated with low frequency acoustic phonons. The stopped muon polarization decays exponentially toward thermal equilioriu with the characteristic spin-1attice 
relaxation tiae constant $T_{1}$.

Now consider a $\mu^{+}$with 1 ts spin anti-parallel to the beam direction. According to $(V-A)$ theory the probabilty that the decay $e^{+}$ is emitted along the beam direction is enhanced by a factor of

$$
E(x)=1 / 2(1-x)
$$

if the muon spin direction is reversed. The decay time spectrum becomes

$$
N(x, t)=N_{0} \exp \left(-t / \tau_{\mu}\right)\left\{\exp \left(-t / T_{1}\right)+E(x)\left[1-\exp \left(-t / T_{1}\right)\right]\right\}
$$

If $T_{1}$ is much longer than the observation time the decay spectrum appears almost exponential with an effective measured muon mean-life $\tau^{\prime}{ }^{\prime}$ given by:

$$
\tau_{\mu}{ }^{\prime}=\frac{\tau_{\mu} T_{1}}{T_{1}-[E(x)-1] \tau_{\mu}}
$$

Thus $\tau_{\mu}{ }^{\prime}>\tau_{\mu}$ for $x>1 / 2$, and the effect increases rapidly as $x \rightarrow 1$.

The spin-held data from the second running period ('Run 2') with $x>0.88$ is shown in Figure (4.1). The fitted muon mean-ilfe is $\tau_{\mu}{ }^{\prime}=2.214 \pm 0.004$ (stat) $\mu$ s and the fitted background of $1.2 \pm 9.8$ per time bin is consistent with zero.

Figure (4.2) shows the $\tau_{\mu}$ ' of the spin-held data fitted as a function of the decay $\mathrm{e}^{+}$momentum. The background, which was found to be consistent with zero throughout the $x$ range, was fixed to zero. The Run 2 and hun 3 aluainum target data has been combined. Different $\mu^{+}$ lifetime clocks were used in each of the three running periods, and the lower statistics Run 1 data has been caltted since 1 covered a shorter $x$ range than the Run 2 and Rur 3 data. The curves are fits to equation (4.1) with finite anguiar acceptance erfects included in $E(x)$ and 


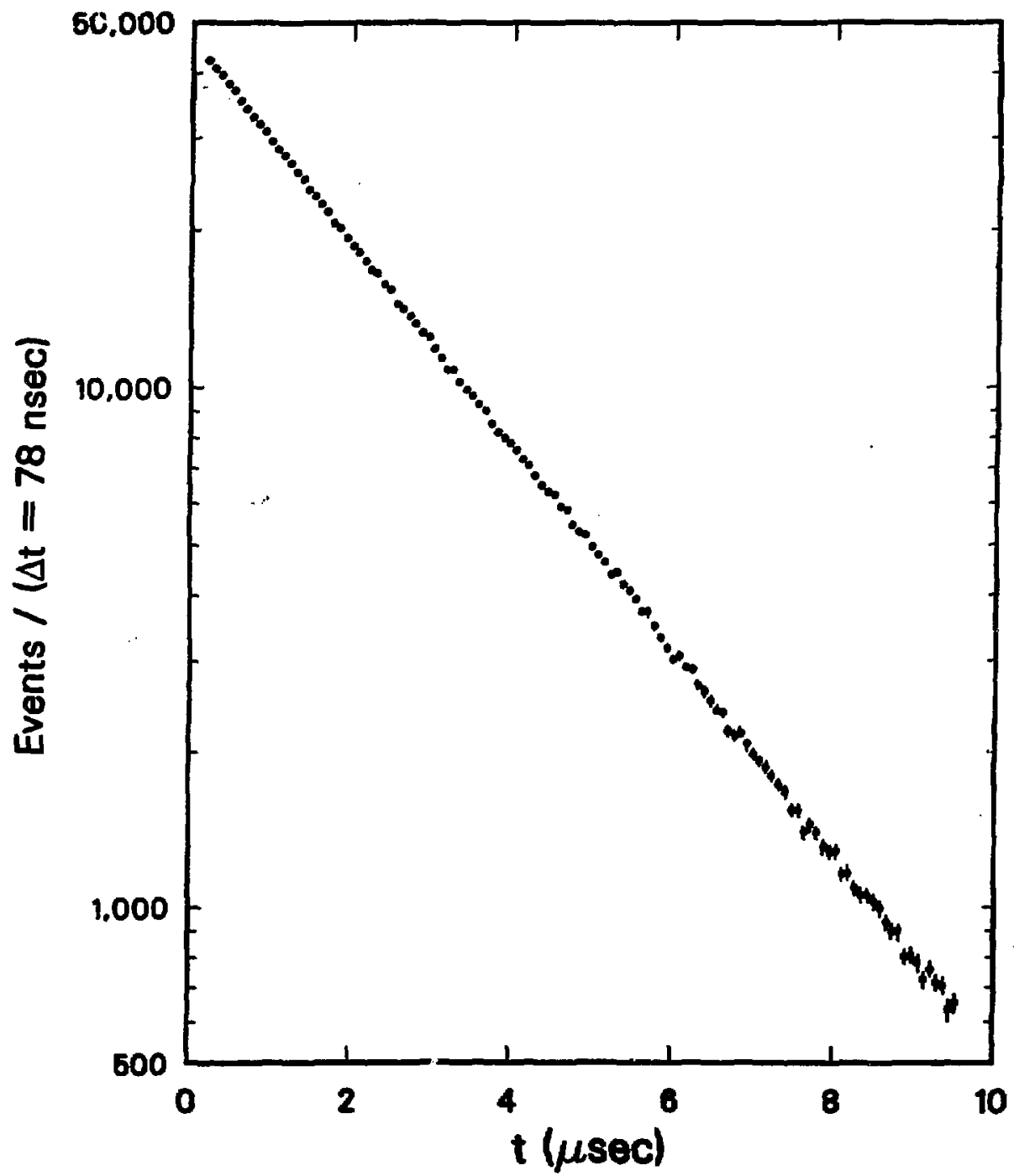

xce 053-16e

FIGURE (4.1). Tine spectrun of the spin-held data from Run 2. The fitted muon man-11fe is $x_{w}-2.214 \pm 0.004(s t a t$.$) wo with a fitted$ backeround of $1.2 \$ 9.8$ per tine bln. 


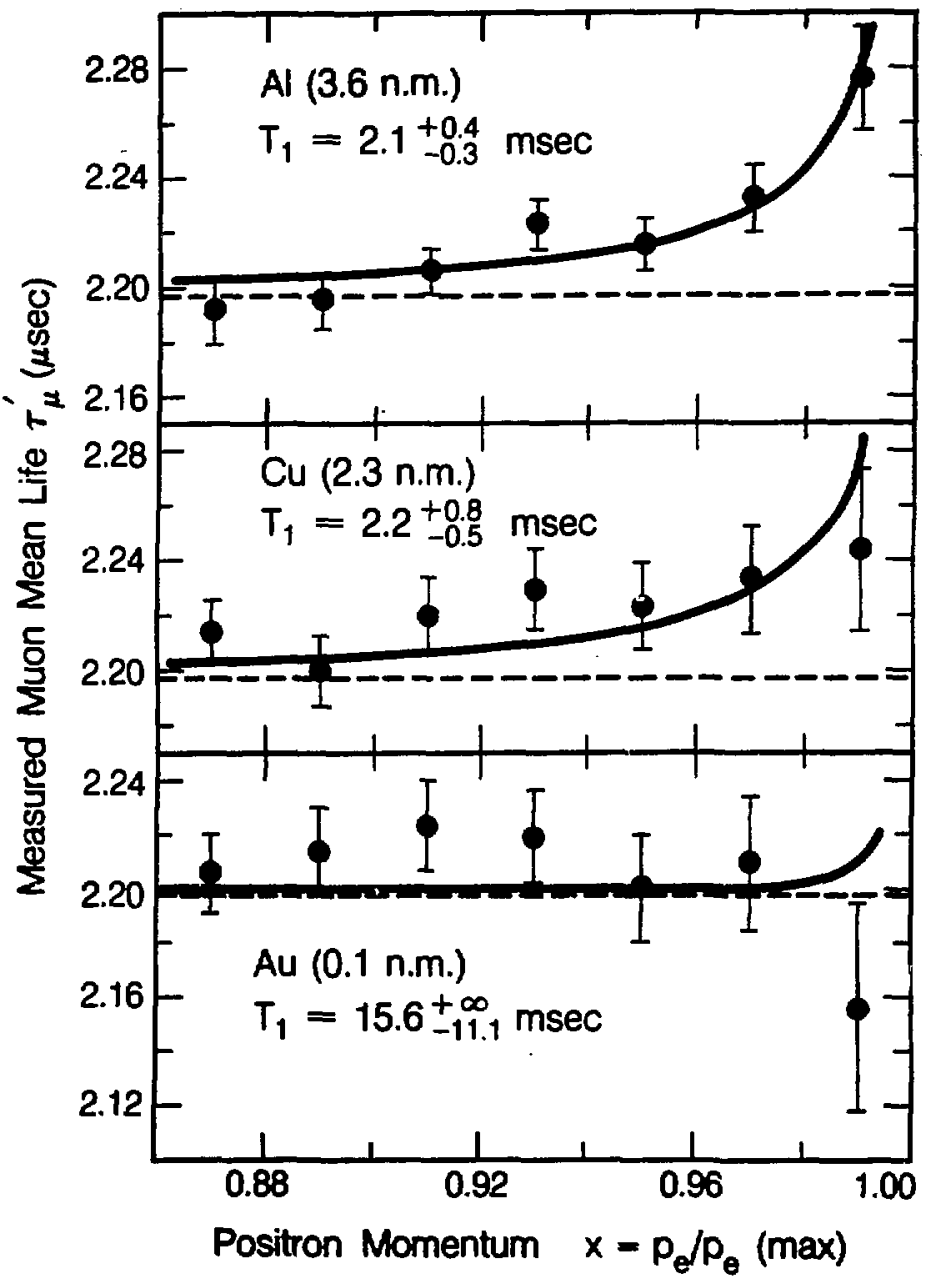

XBL. 658-11663

FICURE (4.2). Fitted won mean-11fe $\tau_{\mu}$ ' versus decay positron womentur for spln-held data from al-inu, copper, and gold targets. The tareet mateidal nuciear agnetic monent in units of nuclear agnetons (n.m., is Indlated. The correlation between the putative apdm-1attice reluxatlon times $T_{1}$ and the nuclear wametic woments surgests a real spifo-lattice relaxation erfect. 
assuming the true muon mean-11fe $\tau_{p}=2.137 \mathrm{\mu s}$. The best fit spin-lattice relaxation time constants of $T_{1}=2.1_{-0.3}^{+0.4}$ ms for $A 1, T_{1}=2.2_{-0.5}^{+0.8}$ ms ror $\mathrm{Cu}$, and $T_{1}=15.6_{-11.1}^{+\infty} \mathrm{ms}$ for Au correlate with their respect 1 ve nuciear dipoie moments of $3.6,2.3$, and $\mathrm{V} .1$ nuclear magnetons. This correlation suggests the effect is due to spin-lattice relaxation rather than some residual background problem. In principle the foregoing method provides 2 means of measuring $\mu^{+}$spin-lattice relaxat on time constants $T_{1}-10^{3} \tau_{j}$.

In conclusion no spin-held data muon lifetime information is used in fitting the $\mu S R$ data, which is time-average unpolarized, since the two data sets do not necessarily have the same apparent $\tau_{\mu}$.

4. 4 Spin-Spin Relaxation: jSh Signal Daniping

The spins of muons stopped in the target material precess under the combined influence of the external transverse magnetic field and the randomly oriented internal local fields produced mainly by the nuclear magnetic dipole inoments. The muon spins therefore frecess with slightly different Larmor frequencles resulting in a loss $0^{\prime}$, ihase coherence.

The decay $o_{1}$ the spin phase coherence is observed experinentally as a damping, $G(t)$, of the $\mu S R$ signal amplitude. This is seen in Figure (4.3) which displays data from the second run period. Although the $\mu S R$ signal damping can yield much information about the environment in which the $\mu^{+}$are brought to rest, it is clearly an unwelcome nuisance In an experiment where one would like to measure a $\mu S R$ sIgnal anilitude deterained solely by the weak interaction. If the exact form of $G(t)$ were known the desired amplitude would, in princlple, be sinply the tine t $\rightarrow$ anplitude obtained from a fit to the uSR data. Unfortunately. 


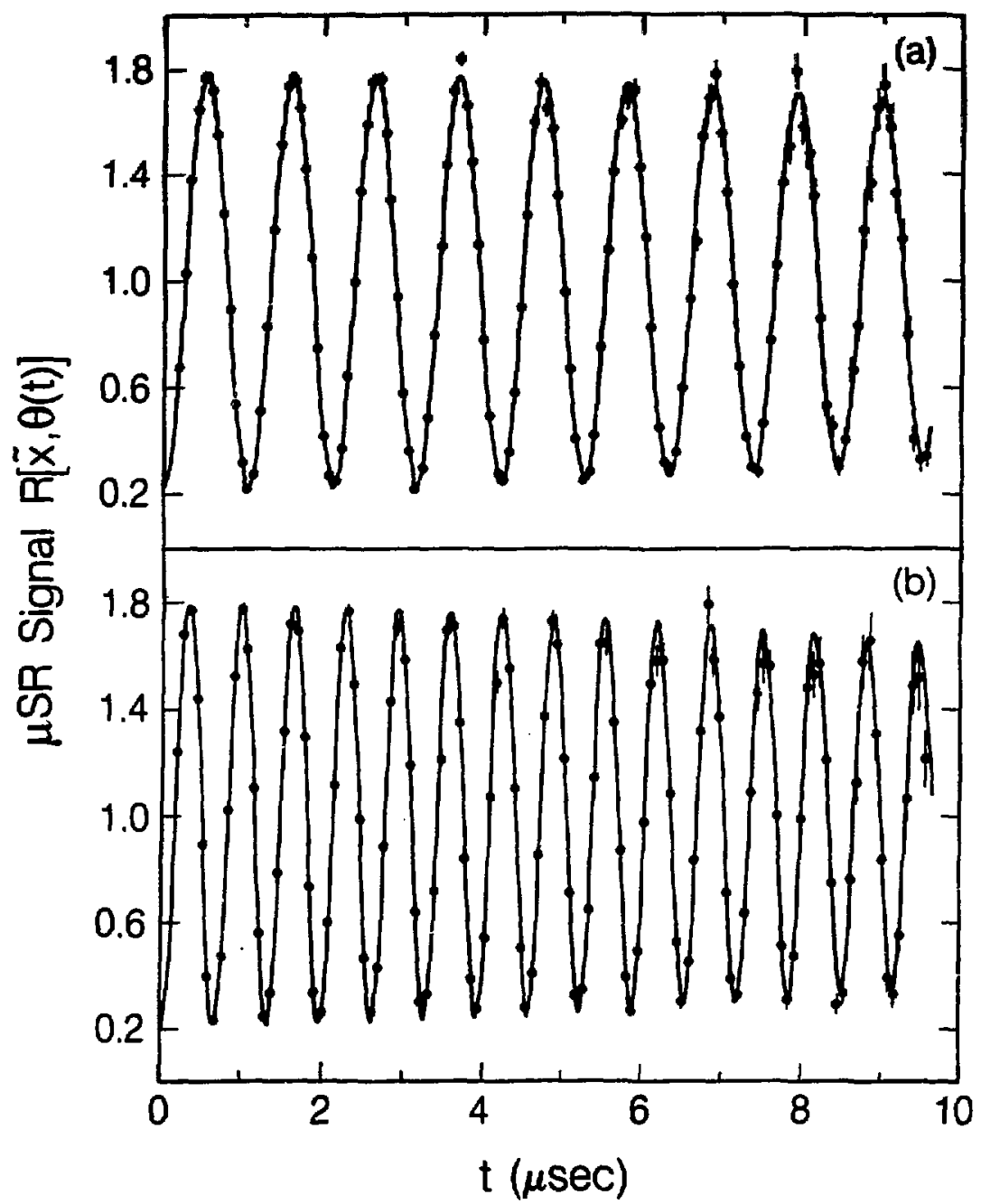

XBL 8412-6004

FIGURE (4.3). The $\mu$ SR data from Run 2, contributing $73 \%$ of the total data base for the final results, with spin-precessing fields (a) $B_{T}=70-G$, and (b) $B_{T}=110-G$. The exponentlal decay with muon lifetime has beei factored out. Spin-spin relaxation causes a damping of the $\mu$ SR st gmal angl ture. 
there is no 'angle foreula' for $G(t)$ which describes exactly the si nal duping observed in real, 1.e. Inperfect, materials such as netals which contaln; to some degree, Inpurities and lattice defects. However, it will be seen in the following ilscussion that approximate expressions for $G(t)$ can be obtained if certain simplifying assumptions are made.

A wealth of general information about spin depolarization in $\mu$ SR experiments can be found in the proceedings of recent topical conferences ${ }^{0}$ ). The recent review of transport wechanisms of light interstitials in metals by Richter ${ }^{41}$ ) sumarizes much useful inforwation:

In metals with large nuclear dipole moments such as copper and aluminum the local dipole fields are a few Gauss at the interstitial lattice sites occupied by the muons. The $\mu^{+}$spin phase coherence decays according to the ensemble average

$$
G(t) \exp \left(i \omega_{0} t\right)=\left\langle\exp \left[1 \int_{0}^{t} \omega\left(t^{\prime}\right) d t^{\prime}\right]\right\rangle
$$

where. $\omega(t)=\omega_{0}+w^{\prime}(t)$ with $w_{0}$ the Larmor frequency in the external field alone and $\omega^{\prime}(t)$ the frequenoy shift due to dipolar interactions. An approximate analytic expression for $G(t)$ can be obtained by assuming (i) that the frequency modulation $\omega^{\prime}(t)$ is random, (ii) that it is a Gaussian random process so that only the second-order cumulant, or correlation function of $w^{\prime}(t)$ with $\omega^{\prime}(v)$, need be considered and (11i) that the correlation function decays exponentially with forrglefign tise $\tau_{c}$ characteristic of the tien a $u^{+}$resldes at a lattice site before diffusing to another. The carrelation function becomes 


$$
\left\langle\omega^{\prime}(t) \omega(0)\right\rangle=\left\langle\omega^{\prime}(0)^{2}\right\rangle \exp \left(-t / \tau_{c}\right)=20^{2} \exp \left(-t / \tau_{c}\right)
$$

and then $\quad G(t)=\exp \left(-20^{2} \tau_{c}^{2}\left[\exp \left(-t / \tau_{c}\right)-1+t / \tau_{c}\right]\right\}$

Equation (4.2) is the Kubo-Tomita42) or motional-narrowing form of the spin relaxation function.

In the limiting case of immobile $\mu^{+} G\left(t, \tau_{c}+\infty\right)=\exp \left(-\sigma^{2} t^{2}\right)$, while for extremely mobile $\mu^{+}$the local fleld fluctuations are averaged and motional-narrowing occurs: $G\left(t, \tau_{c}+0\right)=\exp \left(-2 o^{2} \tau_{c} t\right)$. For intermediate values of $\tau_{c}$ equation (4.2) provides a useful interpolation between the Gaussian and exponential limits.

The static linewidth $0^{2}$ is related to the random local dipole fields $\Delta B$ by

$$
0^{2}=\gamma_{\mu}^{2}\left\langle\Delta B^{2}\right\rangle / 2
$$

where $\gamma_{\mu}=8.5 \times 10^{4} \mathrm{radians} / \mathrm{sec}-G$, and is $\mathrm{gi}$ ven by the van vleck formula ${ }^{43}$ )

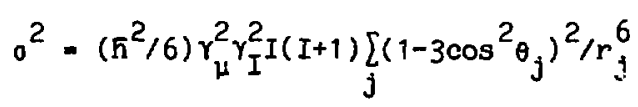

where $r_{j}$ is the distance of the $\mu^{+}$from the nuclear spin $I_{j}, \theta_{j}$ is the angle between $\hat{r}_{j}$ and the external fleld direction, and $\gamma_{\mu}$ and $\gamma_{I}$ are the gyromagnetic ratios for the $\mu^{+}$and nuciel, respectively. According to equacion (4.4) $0^{2}$ depends markedly on the crystal lattice orientation relative to the external field. However, for the small external fields used in the present experiment $(=100 \mathrm{G})$ the orientation dependence is reduced strongly by additional interactions between the nuclear quadrupole moments and the electric field gradient produced by 


\section{the muon.}

The aln shortconings in the assuptions used to obtain $G(t)$ in equation (4.2) are now considered. Kehr et al." ") have shown that inclusion of only the second-order cumulant leads to a more rapid damping than that exhibited by their more general Markovian-randon walk formulation. Although the precession erequency shifts $\omega^{\prime}$ are different at each interstitial site there are correlations between the $\omega$ ' at nelghboring sites because the $\mu^{+}$is subject to some of the same nuclear spins. This effect can be treated approximately by using a correlation time $\tau_{c}$ longer than the mean $\mu^{+}$residence time at each site. In addition, since the $\mu^{+}$has been regarded as a classical particle localized at specific sites, possible delocalization effects have been neglected.

The preceding discussion has also ignored the possibility that $\mu^{+}$ become trapped at lattice defects. The defects may be impurities such as oxygen or nitrogen atoms which trap $\mu^{+}$below about $80 \mathrm{~K}$, lattice vacancies or dislocations which trap $\mu^{+}$up to about room temperature, . or larger volds in which the surface electric dipole layer and image force can produce a deep trapping well ${ }^{45}$ ). Kehr et al. 44) have also constructed a Markovian-random walk theory of spin depolarization for diffusion in the presence of traps. They consider a two state model in which the $\mu^{+}$is either trapped for an average time $\tau_{0}$ during which $G(t)=\exp \left(-o^{2} t^{2}\right)$, which is the simplest approximation correryonding to muons at fixed sites in the traps, or is untrapped for an average time $T_{2}$ during which $G(t)$ is taken to be their result in the absence of traps. The contributing randon walk processes are sumed in integral equations which are solved by taplace transform and inverted 
numerically to yield $G(t)$. It should be noted that the initial

conditions are not equilibrium conditions since the $\mu^{+}$are stopped at random sites. If the concentration of traps is $c$ then at time $t=0$ the fraction of $\mu^{+}$in traps is $c$, while under equilibrium conditions the fraction is $\tau_{0} /\left(\tau_{0}+\tau_{1}\right)$. At room temperature equilibrium shovid be established in times short compared to the mean $\mu^{+}$lifetime.

The observed $\mu$ SR signal damping, in principle, has a small spin-lattice relaxation component. Any non-uniformities in the applied spin-precessing field $B_{T}$ also contribute.

It should now de clear that the $G(t)$ of equation (4.2) can provide only an approximation to the true form of the $\mu \mathrm{SR}$ signal damping. Therefore fitting the $\mu \mathrm{SR}$ data assuming equation (4.2) to be valid may lead to a fitted time $t=0$ amplitude either smaller or larger than the true amplitude. The approach taken in the data analysis discussed in Chapter 7 is to use the Gaussian limit of equation (4.2) and then try to show that this underestimates the true time $t=0$ amplitude. This procedure yields more conservative limits on rlght-handed currents. 


\section{Chapter 5}

\section{The Beanl ine and Apparatus}

\subsection{The Beaml ine}

The TRIUMF M13 beamline $\left.{ }^{4}\right)$ shown in Figure (5.1) is a low monentum (20-130 MeV/c) pion and muon channel viewing the 1AT1 production target at $135^{\circ}$ with respect to the primary proton beam. The secondary beam is transported through two $60^{\circ}$ bends, the first right and the second left, to a final focus (F3) nominally $9.4 \mathrm{~m}$ downstream of the production target. The symmetric quadrupole triplet (Q3-Q5) produces a relative inversion of the Images at the intermediate focl F1 and F2, thereby ylelding an achromatic focus at F3. The symmetric configuration of the beamline elements also suppresses second order effects and produces a magnification of unity at F3. The beam phase space is governed by the setting of the horizontal and vertical jaws (J) upstream of the first dipole (B1). The momentum bite is restricted by the horizontal components of slits SL1 and SL2 at the intermodiate foc1 F1 and F2. With the exception of B1 in Run 1, the dipoles were NMR-monitored.

Figure (5.2) shows the positive particle fluxes obtained in the beam tuning studies of ref. (46). For data collection in the present experiment the beamline was tuned to $29.5 \mathrm{MeV} / \mathrm{c}, 1 . e .18$ below the $29.8 \mathrm{HeV} / \mathrm{c}$ surface muon edge. This allowed a $28 \Delta \mathrm{p} / \mathrm{p}$ momentum bite during occesional periods of low prinary proton flux, although a 15 $\Delta p / p$ was noraally used. Under noral running conditions $100 \mu h$ or 500 HeV protons incldent on a 2 thick carbon production target yielded $1.8 \times 10^{4} p^{+} /$sec at the stopping target. The $\mu^{+}$bem spot res spatial and angular dinensions were typloally 6 mand 35 arad 


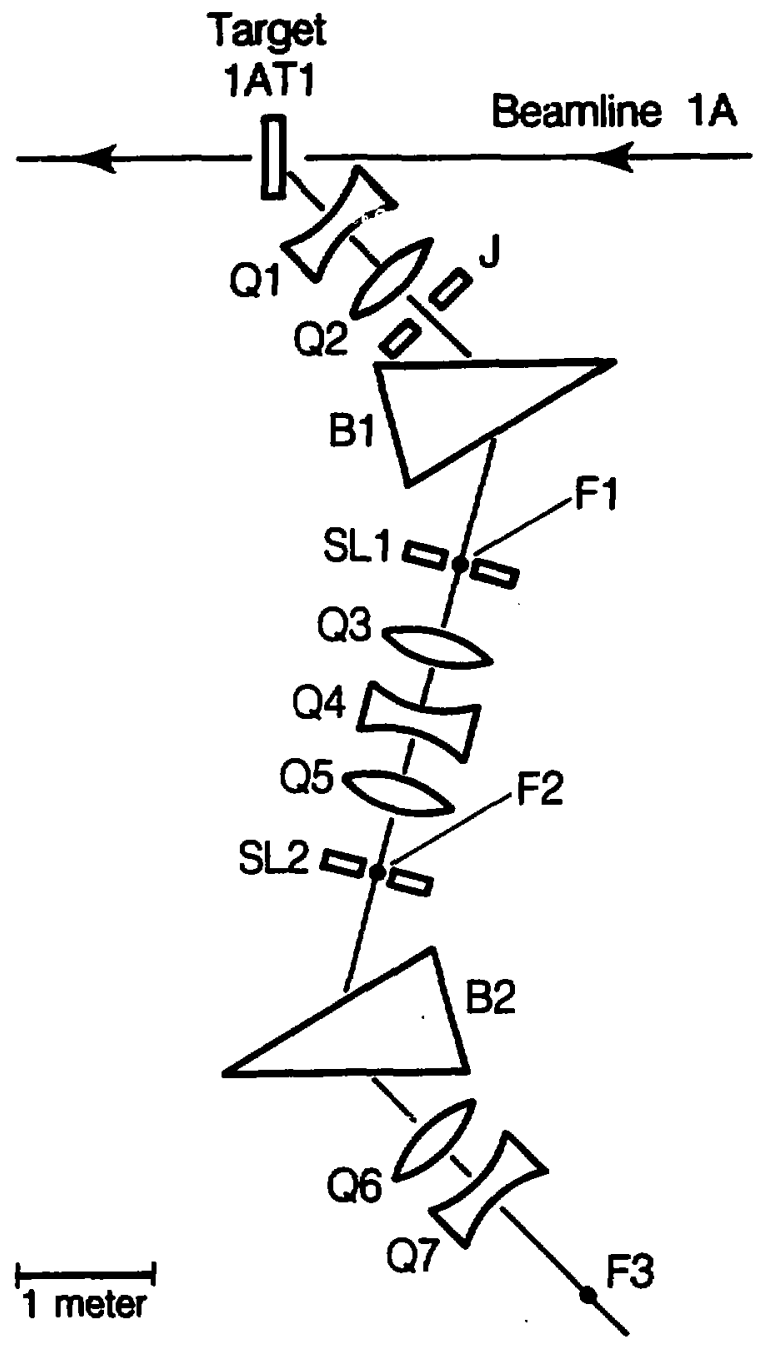

XBL 858.11662

FICURE (5.1). The TRIURF H13 beanl ine. B1 and B2 are dipoles; Q1-Q7 are quadrupoles; F1-F3 are foci; the sl1ts SL1 and SL2, and the jaws J have both horizontal and vertioul components. 


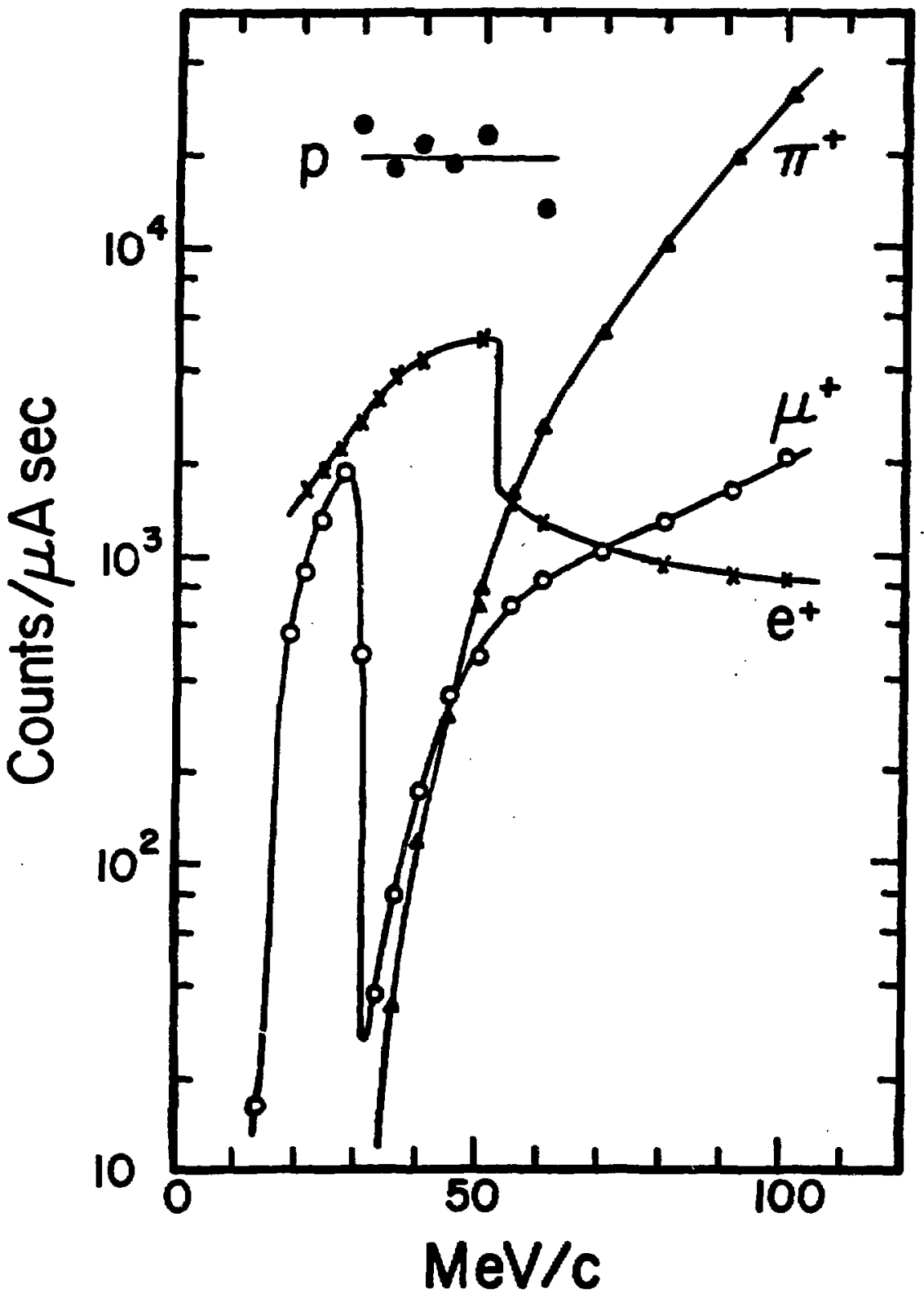

FIGURE (5.2). Particle fluxes versus bealine nonentu setting (taken from ref. 16 ). 
horizontally, and $5 \mathrm{~mm}$ and 70 mrad vertically.

Beam $e^{4}$ pass through the stopping target and do not satisfy the trigger requirements. Beam protons are stopped far upstream, mostly in the beamline vacuum window. The pulsed nature of the primary proton beam allows prompt $\pi^{+}$and $\mu^{+}$from $\pi^{+}$decay in flight to be eliminated by timing cuts relative to the cyclotron $\mathrm{rf}$ cycle.

Approximately $2 \%$ of the $\mu^{+}$flux originates from $\pi^{+}$decay in flight. These 'cloud' $\mu$ ' are, on average, far less polarized than the surface muons. As an extreme example, the (V-A) backward decay of an $81.0 \mathrm{MeV} / \mathrm{C}$ $\pi^{+}$yields a forward moving $29.5 \mathrm{MeV} / \mathrm{C} \mu^{+}$with parallel spin and momentum directions, thereby mimicking a $(V+A)$-produced surface muon. However, efficient transport of cloud muons to tile stopping target beam spot (F3) requires the in-flight $\pi^{+}$decays to occur close to the production target, i.e. to be prompt. The primary protons arrive at the production target in bursts of 2-5 nsec duration $43 \mathrm{nsec}$ apart. In Figure (5.3)(a) the exponential decay of $\pi^{+}$at rest $\left(\tau_{\pi}=23\right.$ nsec) underlies the time distribution, relative to the cyclotron $r f$ cycle, of $29.5 \mathrm{MeV} / \mathrm{C} \mu^{+}$arriving at the stopping target. The residual cloud $\mu^{+}$ and prompt $\pi^{+}$peaks are clearly visible in the Figure (5.3)(b) arrival times of $30.5 \mathrm{MeV} / \mathrm{c}$ beam particles. Events with beam particle arrival tim: 3 in the shaded regions of F1gure (5.3), which contain $98 \%$ of the cloud $\mu^{+}$, are rejected in the data analysis.

\subsection{The Apparatus: An Overview}

After traversing the beamline the bean passed through a 2 all nylar vacum whow and entered the apparatus shown in Figure (5.4). Bean $u^{*}$ were stopped in elther a metal foll or 11 quid mellum target positioned 

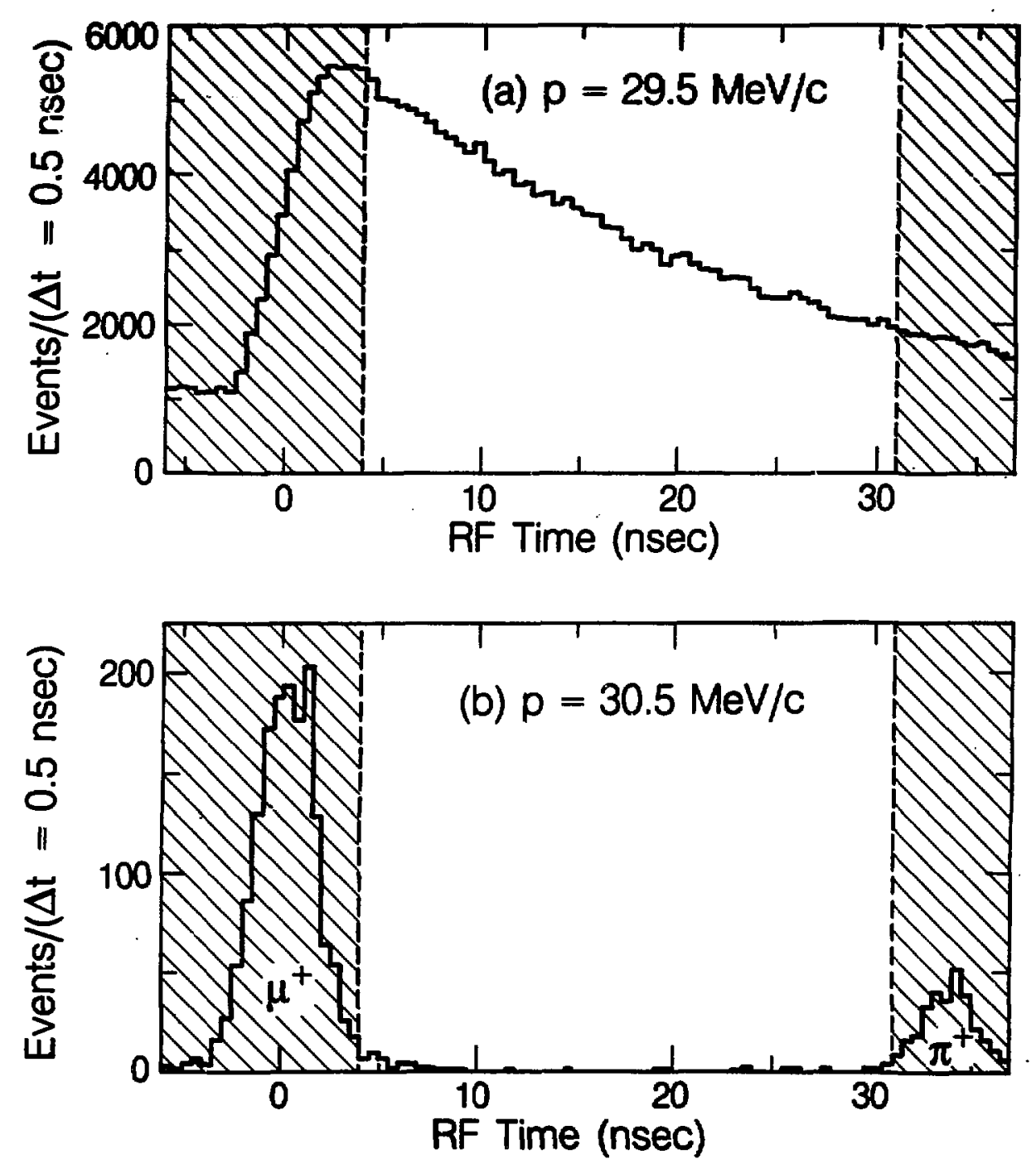

XBL 859-11664

FIGURE (5.3). Bean particle arrival tines with respect to the $43 \mathrm{~ns}$ cyclotron $r f$ cycle at (a) $29.5 \mathrm{MeV} / \mathrm{c}$ and (b) $30.5 \mathrm{KeV} / \mathrm{c}$. The shaded regions rontain alwost all of the cloud $\mu^{*}$ and prompt ${ }^{*}$ contalinations and are rejected. 


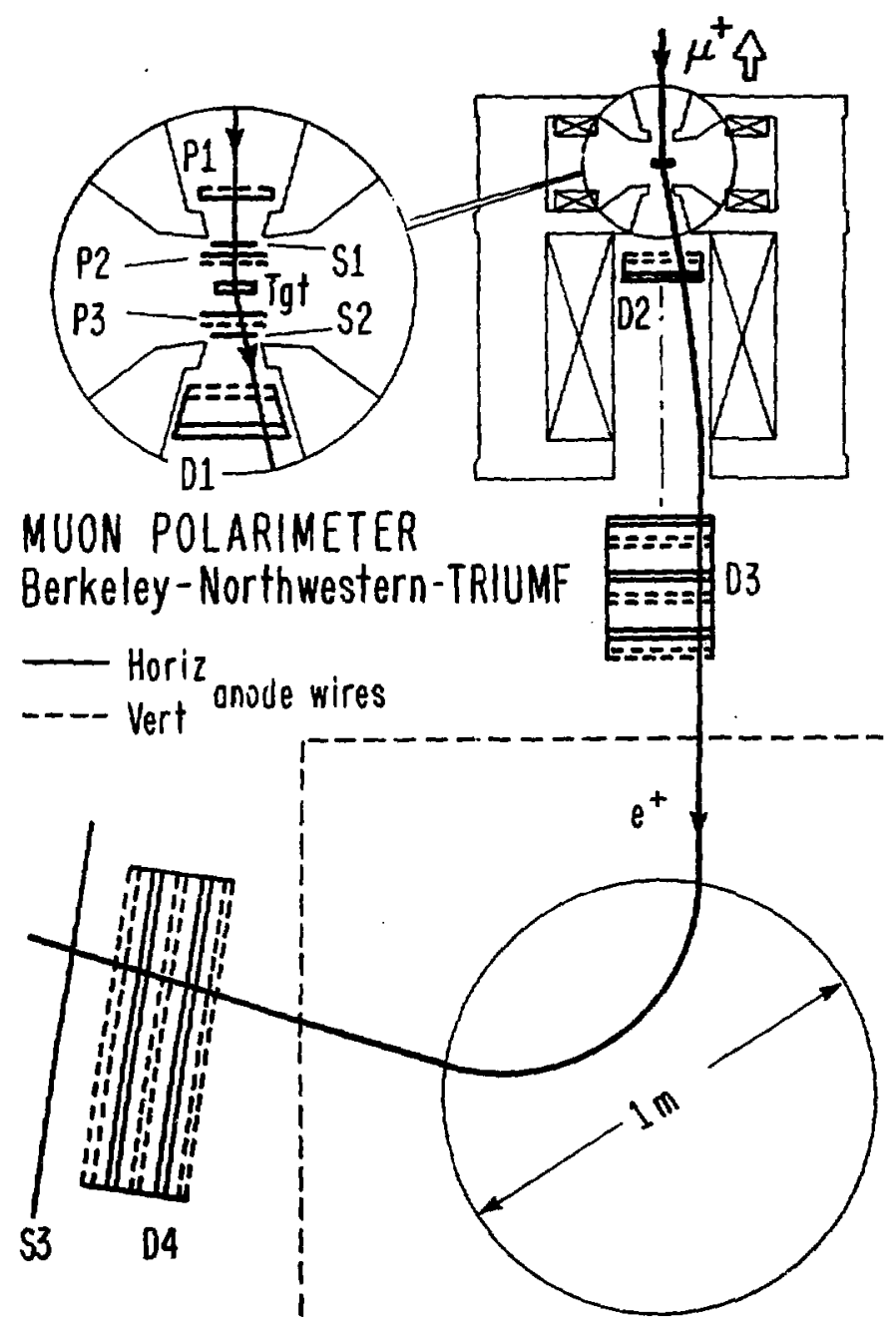

XEL E34.147

FICURE (5.4). The apparatus. P1-P3 are proportional chambers; S1-S3 are scintillators; DA-D4 are drift chanbers. Muons entering the solenold are stopped in the carget (7et). Decay $e^{*}$ enitted near the beam direction are focused oy the solenold into the spectraneter. 
at the center of the upstream section of the solenold. The amount of material upstream of the stopping target was estimated to be $50 \mathrm{mg} / \mathrm{cm}^{2}$ In Run $1,54 \mathrm{mg} / \mathrm{cm}^{2}$ in Run 2, and $55 \mathrm{mg} / \mathrm{cm}^{2}$ in Run 3 .

Decay $\mathrm{e}^{+}$emitted within $270 \mathrm{mrad}$ of the beam direction were focused by the downstream section of the solenoid into a horizontally focusing cylindrical dipole spectrometer for momentum analysis. Multiwire proportional chambers and drift chambers in the target region measured the incoming beam $\mu^{+}$and outgoing decay $e^{+}$trajectories. Tracks recorded by drift chambers located near the conjugate foci of the spectrometer allowed reconstruction of the decay $\mathrm{e}^{+}$momentum. The amount of material downstream of the stopping target and upstream of the spectrometer was estimated to be $186 \mathrm{mg} / \mathrm{cm}^{2}$ in Run $1,193 \mathrm{mg} / \mathrm{cm}^{2}$ in Run 2, and $216 \mathrm{mg} / \mathrm{cm}^{2}$ in Run 3 .

\subsubsection{The Solenoid}

The solenoid consists of two co-axial sections essentially decoupled by the intervening septum. The two water-cocied coils of the upstream section produce the longitudinal field for the spin-held mode of the experiment. They have inner diameter 6", outer diameter 10", length 2", 29 turns/coll, and a center-to-center separation of $7^{n}$. The pole faces and coll separation were designed to mininize radial field components over the target repion. Conputer simulations using the propa poissol indicated that within a radius of 1 " and within \pm 0.25 " longitudinally of the noainal tareet position the fleld direction is axial to within 2 mrad.

The vertlcal transwerse field used in the uSh wode was produced by 
an additional water-cooled coil. The $\mu S R$ coil consisted of a single turn of $0.125^{\prime \prime} \times 0.5^{\prime \prime}$ copper having four horizontal sections transverse to the bean direction with centers $1.125^{\prime \prime}$ above and below the beam axis and 1.54" upstream and downstream of the nominal target position. Studies using the program POISSON indicated that within \pm 1 " of the beam axis and within $\pm 0.7 "$ longitudinally of the target position the longit dinal field component did not exceed $1.0 \%$ of the transverse field. Field measurements made with the coll outside the solenoid Indicated field strength uniformity of $\pm 0.4 \%$ within 0.75 " of the beam axis at the nominal target position. Transverse fields of $70-G$ and 110-G were obtained with coll currents of $475-A$ and $750-A$, respectively. A residual longltudinal field of about $40-G$ remaining at the target position after the upstream longitudinal field colls were turned off was nuiled to within 2-G by applying a small reverse current to the colls. The null condition was indicated by a maximal ratio of events to stopped $\mu^{+}$in Run 1, and by field measurements in Runs 2 and 3.

The downstream section of the solenoid has three colls each with inner diameter 4.5", outer diameter 10", longth $6.25^{n}$ and 120 tunns/coil.

Table (5.1) shows the on-axis longitudinal fields calculated by POISSCN for the spin-held $\left(B_{1}\right)$ and $\mu S R\left(B_{T}\right)$ modes. The stopning target position is at zero, with downstream positions being positive. The rleld values assume $1.31 \times 10^{5}$ A-turns/coll downstrean, and $5.45 \times: 0^{4}$ A-turns/coll upstrean for $E_{i}$. For $2211.25{ }^{m} B_{L 2}{ }^{*} B_{T Z}$. 


\begin{tabular}{|c|c|c|c|c|c|c|c|}
\hline $\begin{array}{c}z \\
\text { (inch) }\end{array}$ & $\mathrm{B}_{\mathrm{Lz}}(\mathrm{z})$ & $\underset{\text { zuss) }}{\mathrm{B}_{\mathrm{Tz}}(\mathrm{z})}$ & $\stackrel{2}{(\text { Inch })}$ & $\mathrm{B}_{\mathrm{Lz}}(\mathrm{z})$ & $\begin{array}{l}\mathrm{B}_{\mathrm{Tz}}(\mathrm{z}) \\
\text { uss) }\end{array}$ & $\begin{array}{c}z \\
\text { (inch) }\end{array}$ & $\begin{array}{r}\mathrm{J}_{\mathrm{Tz}}(\mathrm{z}) \\
\text { (Gauss) }\end{array}$ \\
\hline $\begin{array}{l}-4.25 \\
-4.00 \\
-3.75 \\
-3.50 \\
-3.25 \\
-3.00 \\
-2.75 \\
-2.50 \\
-2.25 \\
-2.00 \\
-1.75 \\
-1.50 \\
-1.25 \\
-0.75 \\
-0.50 \\
-0.25 \\
0.00 \\
0.25 \\
0.50 \\
0.75 \\
1.00 \\
1.25 \\
1.50 \\
1.75 \\
2.00 \\
2.25 \\
2.50 \\
2.75 \\
3.00 \\
3.25 \\
3.50 \\
3.75 \\
4.00 \\
4.25 \\
4.50 \\
4.75 \\
5.00 \\
5.25 \\
5.50 \\
5.75 \\
6.00 \\
6.25\end{array}$ & $\begin{array}{r}283 \\
388 \\
528 \\
719 \\
1028 \\
1538 \\
2385 \\
3627 \\
5429 \\
7358 \\
9035 \\
10268 \\
11014 \\
11654 \\
11754 \\
11801 \\
11811 \\
11805 \\
11763 \\
11668 \\
11450 \\
11038 \\
10300 \\
9079 \\
7421 \\
5523 \\
3765 \\
2587 \\
1821 \\
1406 \\
1214 \\
1170 \\
1208 \\
1323 \\
1525 \\
1803 \\
2146 \\
2564 \\
3059 \\
3596 \\
4184 \\
4805\end{array}$ & $\begin{array}{r}0 \\
1 \\
2 \\
3 \\
6 \\
11 \\
21 \\
34 \\
56 \\
89 \\
138 \\
206 \\
290 \\
387 \\
505 \\
654 \\
831 \\
1050 \\
1321 \\
1649 \\
2030 \\
2475 \\
2990 \\
3542 \\
4142 \\
4772\end{array}$ & $\begin{array}{r}6.50 \\
6.75 \\
7.00 \\
7.25 \\
7.50 \\
7.75 \\
8.00 \\
8.25 \\
8.50 \\
8.75 \\
9.00 \\
9.25 \\
9.50 \\
9.75 \\
10.00 \\
10.25 \\
10.50 \\
10.75 \\
11.00 \\
11.25 \\
11.50 \\
12.00 \\
12.50 \\
13.00 \\
13.50 \\
14.00 \\
14.50 \\
15.00 \\
15.50 \\
16.00 \\
16.50 \\
17.00 \\
17.50 \\
18.00 \\
18.50 \\
19.00 \\
19.50 \\
20.00 \\
20.50 \\
21.00 \\
21.50 \\
22.00\end{array}$ & $\begin{array}{l}5439 \\
6034 \\
6569 \\
7081 \\
7522 \\
7894 \\
8217 \\
8491 \\
8721 \\
8913 \\
9073 \\
9204 \\
9315 \\
9406 \\
9480 \\
9533 \\
9580 \\
9617 \\
9648 \\
9674\end{array}$ & $\begin{array}{l}5412 \\
6011 \\
6550 \\
7064 \\
7507 \\
7881 \\
8205 \\
8480 \\
8711 \\
8903 \\
9064 \\
9195 \\
9307 \\
9399 \\
9473 \\
9526 \\
9573 \\
9611 \\
9642 \\
9668 \\
9690 \\
9728 \\
9755 \\
9771 \\
9788 \\
9794 \\
9794 \\
9788 \\
9782 \\
9770 \\
9758 \\
9738 \\
9712 \\
9676 \\
9637 \\
9588 \\
9531 \\
9458 \\
9367 \\
9251 \\
9110 \\
9932\end{array}$ & $\begin{array}{l}22.25 \\
22.50 \\
22.75 \\
23.00 \\
23.25 \\
23.50 \\
23.75 \\
24.00 \\
24.25 \\
24.50 \\
24.75 \\
25.00 \\
25.25 \\
25.50 \\
25.75 \\
26.00 \\
26.25 \\
26.50 \\
26.75 \\
27.00 \\
27.25 \\
27.50 \\
27.75 \\
28.00 \\
28.25 \\
28.50 \\
28.75 \\
29.00 \\
29.25 \\
29.50 \\
29.75 \\
30.00 \\
30.25 \\
30.50 \\
30.75 \\
31.00 \\
31.50 \\
32.00 \\
32.50 \\
33.000 \\
33.50 \\
34.00 \\
36.00\end{array}$ & $\begin{array}{l}8828 \\
8730 \\
8600 \\
8454 \\
8291 \\
8110 \\
7910 \\
7691 \\
7451 \\
7191 \\
6912 \\
6611 \\
6293 \\
5956 \\
5610 \\
5256 \\
4895 \\
4527 \\
4168 \\
3821 \\
3483 \\
3159 \\
2847 \\
2559 \\
2295 \\
2048 \\
1819 \\
1613 \\
1428 \\
1260 \\
1109 \\
974 \\
855 \\
749 \\
656 \\
572 \\
435 \\
333 \\
259 \\
220 \\
174 \\
139 \\
65\end{array}$ \\
\hline
\end{tabular}




\subsubsection{The Spectraneter}

The spectrometer consisted of an NM-aonitored horizontalis focusing cylindrical dipole magnet with drift chambers located near its conjugate foci. The magnet was originally used by Sagene et al."7) in measurements of the muon decay $\rho$ parameter. The flat pole faces have a diameter of $37^{n}$ and were separated by a gap of $14.5^{n}$. When operated at 125-A the water-cooled coils produced a central field of $0.32-\mathrm{T}$, a $98^{\circ}$ bend angle for $x=1$ decay $e^{+}$, and a momentum dispersion of $1.07 \% / \mathrm{cm}$. Enclosing the particle trajectories by a vacuum box with 5 mil mylar vacuum windows positioned close to the conjugate focal planes minimized moment um resolution loss due to multiple Coulomb scattering. Drift chambers D3 and D4 [Figure (5.4)] were mounted to the vacuum box immediately upstream and downstream of the vacuum windows, repectively.

\subsubsection{Proportional Chambers}

The proportional chambers P1, P2, and P3 each had one horizontal and one vertical wire plane separated by a grounded $0.5 \mathrm{mil}$ double-side aluminized mylar sheet. The anode wires were 0.5 mil diameter goldplated tungsten with $2 \mathrm{~mm}$ spacing. Cathode signals obtained from the 0.5 mil single-side aluminized mylar chamber windows were used in the trigger.

Chamber P1 had clrcular aperture and 32 wires per plane. The wIndows and ground plane were 4 wn from the wire planes. Chambers PE and P3 were of Identical construction with square aperture and 30 wires per plane. The windows and ground plane were 2 from the wire planes. 
wethylal, and in Run 2 andic cas: 69.75 argon, $30.0 \%$ 1sobutane and 0.35 Ireon.

The operating voltages, applied to the wires, were 3500 y for P1 and 2500 V for P2 and P3 when using nethane/methylal; and 2950 y for PI and 2050 V for P2 and P3 when using maglc gas. Ampliflers for the wire and cathode signals were positioned close to the chambers. The gean efficlency of the wire planes was $>99.5 \%$ per plane.

An additional chamber, denoted ' $A$ ' and identical to P2 and P3, was positioned between $P 1$ and $P 2$ in Run 3 in preparation for a measurement of the decay parameter $\delta$ where the extended data momentum range (20-53 MeV/c) made highly efficient rejection of 'straight-through' beam $\mathrm{e}^{+}$events essential.

\subsubsection{Drift Chambers}

The planar drift chambers D1-DH [F1gure (5.4)] were composed of sub-units each containing two planes of horizontal or vertical sense wires. The sense planes were staggered by a half cell width to resolve left-right ambiguities. The cell geometries used in D1, D2 and D3, and D4 are shown in Figure (5.5). The sense wires were 0.5 mil diameter gold-plated tungsten and the field wires were $3 \mathrm{mil}$ diameter beryliun-copper.

D1 was of conical geonetry. The wire spacing within each plane was $0.400^{n}$ and the spacing between planes was C. $35^{H}$. In downstream order the two vertical and two horizontal sense planes contained $3,4,4$ and 5 wires. The chamber windows were 0.5 mil aluminu.

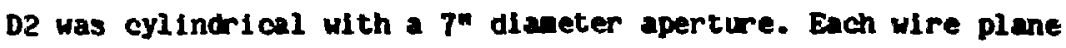


$\Gamma \cdot 1$

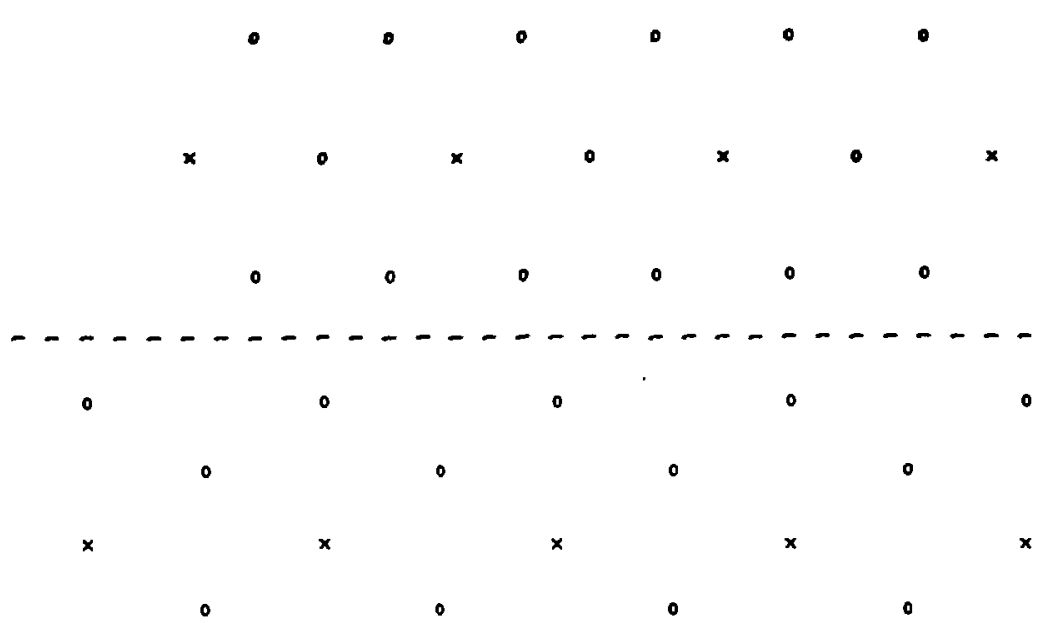

D2, D3

0

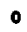

$0 \quad 0$

$\circ$

$\times$

$\times$

$\times$

$x$

$\begin{array}{lllll}0 & 0 & 0 & 0 & 0\end{array}$

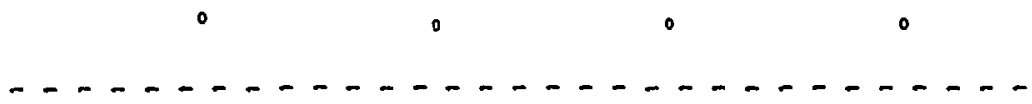

$\mathbf{0}$

0

-

o

。

o

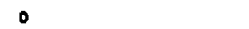

-

o

$x$

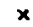

$0 \quad x$

- $x$

$x$

D4

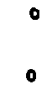

$\circ$

。

。

-
0
0

$\begin{array}{ll}0 & \\ 0 & x \\ 0 & 0\end{array}$

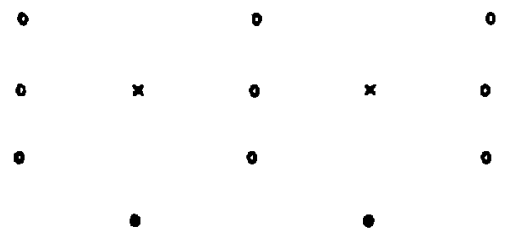

FIGURE (5.5). Drift-chamber cell geometries: sense, - field. 
contained 8 ulres spaced by $0.875^{\circ}$. The separation between planes was $0.250^{\%}$. The ahortest, and potentially least efricient, edge wire in each sense plane was 3 ail beryliu-copper to render it completely inactive. The chamber windows were 0.5 mil alminized aylar.

D3, located at the spectrometer entrance, consisted of 3 cylindrical chambers simllar to D2 except that the aperture diameter was $11^{\prime \prime}$ and there were 12 wires per plane. D3 thus had a total of 6 vertical and 6 horizontal sense planes. The three chambers were separated, except for a narrow outer annulus, by 0.25 mil aluminized mylar windows.

D4, located at the spectrometer exit, had a rectangular aperture and contalned a total of $6 \mathrm{planes}$ of 32 vertical sense wires and 4 planes of 24 horizontal sense wires. The sense wire spacing was 24 mm.

The drift chamber gas was $92 \%$ methane/8\% methylal. The chamber high voltage was applied to the sense wires of D1, and to the fleld wires of D2, D3 and D4. The operating voltages were $+2900 \mathrm{~V}$ for D1, $-2900 \mathrm{~V}$ for D3, and $-3000 \mathrm{~V}$ for D2 and D4. The efficiencies of the sense planes was equalized by applying $+260 \mathrm{~V}$ to the sense planes closest to the chamber windows of $\mathrm{D} 2, \mathrm{D} 3$, and $\mathrm{D} 4$.

Chamber slgnals above a $250 \mu \mathrm{V}$ threshold were amplifled by shielded LeCroy Model 4292 amplifier/discriminator cards mounted close to the chambers. Each chamber had a mean efficiency of at least $97 \$$ per plane except in Run 1, where D1 and D2 had mean efriclencles of $77 \%$ and 838 per plane respectively. 


\section{2 .5 Se1ni:Ilators}

Scintillators S1 and S2 were $5 \mathrm{mil}$ and 10 all NEI02A, respectively. Just upstrean or $S 1$ and downstream of $S 2$ were veto scintillators $V I$ and v2 each of thickness $0.125^{\prime \prime}$ and inner diameter $1.5^{n}$.

Scintillator S3, which covered the downstream area of drift chamber D4 consisted of 3 horlzontal strips $39^{n}$ long $\times 8^{n}$ high $\times 0.375^{\text {n }}$ thick.

S1, S2, V1, V2 and each strip of $S 3$ were viewed from left and right by photomultipliers.

\subsubsection{Stopping Targets}

The muons were stopped in metal foils of $>99.99 \%$ purity or in liquid He. Because folls of optimum thickness were unavaflable the stopping targets were composite, consisting either of two back-to-back foils or a single foll preceded and followed by 1 mil aluminum foils.

The stopping target thicknesses are tabulated in Table (5.2). The compositions of targets having $1 \mathrm{mil}$ Al folls are listed in upstream to downstream order. The target materlal calculated to be encountered by decay $\mathrm{e}^{+}$emitted by a mean range $\mu^{+}$is listed as 'residual thickness.' The residual thickness is also tabulated in terms of calculated $\mu^{+} \mathrm{rms}$ range straggling lengths. The effect of the $1 \% \Delta \mathrm{p} / \mathrm{p}$ momentum bite has been included. Column (a) gives the number of straggling lengths to the downstream surface of the target. Column (b) gives the number of straggling lengths to the closest interface between foils, the + (-) sign indicating that mean range $\mu^{+}$stop beyond (before) the interface. Comparison of the calculated ranges with an experinental range curve taken in Run 2 indicates that the error on the number of straggling 


\begin{tabular}{|c|c|c|c|c|c|c|c|c|c|}
\hline \multirow{2}{*}{$\frac{\text { Target }}{\text { Ag }}$} & \multirow{2}{*}{$\begin{array}{c}\text { Run } \\
1\end{array}$} & \multicolumn{3}{|c|}{$\begin{array}{l}\text { Thickness } \\
\text { (ng/ca') }\end{array}$} & \multicolumn{3}{|c|}{$\begin{array}{c}\text { Residual Thicleness } \\
\left(a g^{2}\right)\end{array}$} & \multicolumn{2}{|c|}{$\begin{array}{c}\text { Residual Stragel ing } \\
\text { Lengths }\end{array}$} \\
\hline & & $\begin{array}{r}2 \times \\
\text { Total }\end{array}$ & $\begin{array}{l}136.5 \\
273\end{array}$ & & & 96 & & 8.1 & $3.4^{+}$ \\
\hline$A 1$ & $1,2,3$ & & 150 & & & 35 & & 4.6 & $3.7^{-}$ \\
\hline$A 1^{*}$ & 2 & $\begin{array}{r}2 x \\
\text { Total }\end{array}$ & $\begin{array}{l}142.5 \\
285\end{array}$ & & & 171 & & 22.6 & $3.6^{+}$ \\
\hline $\mathrm{Au}$ & 1,2 & Total & $\begin{array}{c}6.6 \\
193 \\
6.6 \\
239\end{array}$ & $\begin{array}{l}\mathrm{Al} \\
\mathrm{Au} \\
\mathrm{Al}\end{array}$ & Total & $\begin{array}{c}53 \\
6.6 \\
60\end{array}$ & $\begin{array}{l}\mathrm{Au} \\
\mathrm{Al}\end{array}$ & 4.1 & $3.4^{-}$ \\
\hline $\mathrm{Cu}$ & 2 & Total & $\begin{array}{c}6.6 \\
110 \\
6.6 \\
156\end{array}$ & $\begin{array}{l}\mathrm{Al} \\
\mathrm{Cu} \\
\mathrm{Al}\end{array}$ & Total & $\begin{array}{c}16 \\
6.6 \\
23\end{array}$ & $\begin{array}{l}\mathrm{Cu} \\
\mathrm{Al}\end{array}$ & 2.5 & $1.6^{-}$ \\
\hline $\mathrm{Cu}^{*}$ & 1,2 & $\begin{array}{r}2 x \\
\text { Total }\end{array}$ & $\begin{array}{l}111 \\
222\end{array}$ & & & 81 & & 8.3 & $3.1^{+}$ \\
\hline $\mathrm{He}$ & 1 & Total & $\begin{array}{r}38 \\
150 \\
38 \\
226\end{array}$ & $\begin{array}{l}\mathrm{Al} \\
\mathrm{He} \\
\mathrm{He}\end{array}$ & Total & $\begin{array}{r}86 \\
38 \\
124\end{array}$ & $\begin{array}{l}\text { He } \\
\text { Al }\end{array}$ & 17.2 & \\
\hline
\end{tabular}

Table (5.2)

lengths is unlikely to exceed \pm 0.5 . The $\mathrm{Ag}$ and He targets were used only in Run 1. The residual thlcknesses and straggling lengths for the other targets apply to kun 2. The change of proportional chamber gas from methane/methylal to magic gas for Run 2 and the presence of an additional proportional chamber upstream of the target in Run 3 alter the residual thicknesses. In particular for the $\mathrm{\Lambda 1}, \mathrm{Au}$ and $\mathrm{Cu}$ targets in Ru 1 the number of residual straggling lengths in colun (a) should be reduced by 0.5 , and reduced (Increased) in colum (b) for a - (+) 
sien. For hun 3 the number of residual straggling lengths for the al target should be increased by 0.1 in both colums (a) and (b).

Muons stopping in the air between or beyond the foils, or in the folls' oxidized surface layers are likely to form muonium and depolarize. Column (b) indicates that the $\mathrm{Cu}$ target is too thin. The other targets most likely to have thlckness problems are $\mathrm{Cu}^{*}$ in Run 2 and $\mathrm{Au}$ in Run 1.

\subsection{The Trigger}

The essential features of the trigger logic as it existed in Run 1 are shown in Figure (5.6). Changes made to the logic in Runs 2 and 3 are described later in this section.

The inputs to the trigger logic were signals from the proportional chamber (P1-P3) cathodes, scintiliators (S1-S3) and scintillator vetos (V1, v2) described in the preceding sections and shown in Figure (5.4). The notation PIU, PIV etc. denotes the cathodes assoclated with the wire planes measuring the horizontal and vertical track positions respectively. $S 1 L$ and S1R etc. denotes photomultipliers viewing the scintillators from left and $r$ ight repectively. The three horizontal scintillator strips of $S 3$ were viewed froin left and right, and in top to bottom order, by photomultipliers denoted by $(01, G 4),(62,05)$, and $(G 3, G 6)$.

Three triggers were used: the stral ght-through trigger for spectrometer mcmentum calibration with beam $e^{+} ;$the $\mu$-decay trigger for normal data taking; and the pulser trigger for online diagnostics such as checking $A D C$ pedestals and searching for 'not" or osclllating 


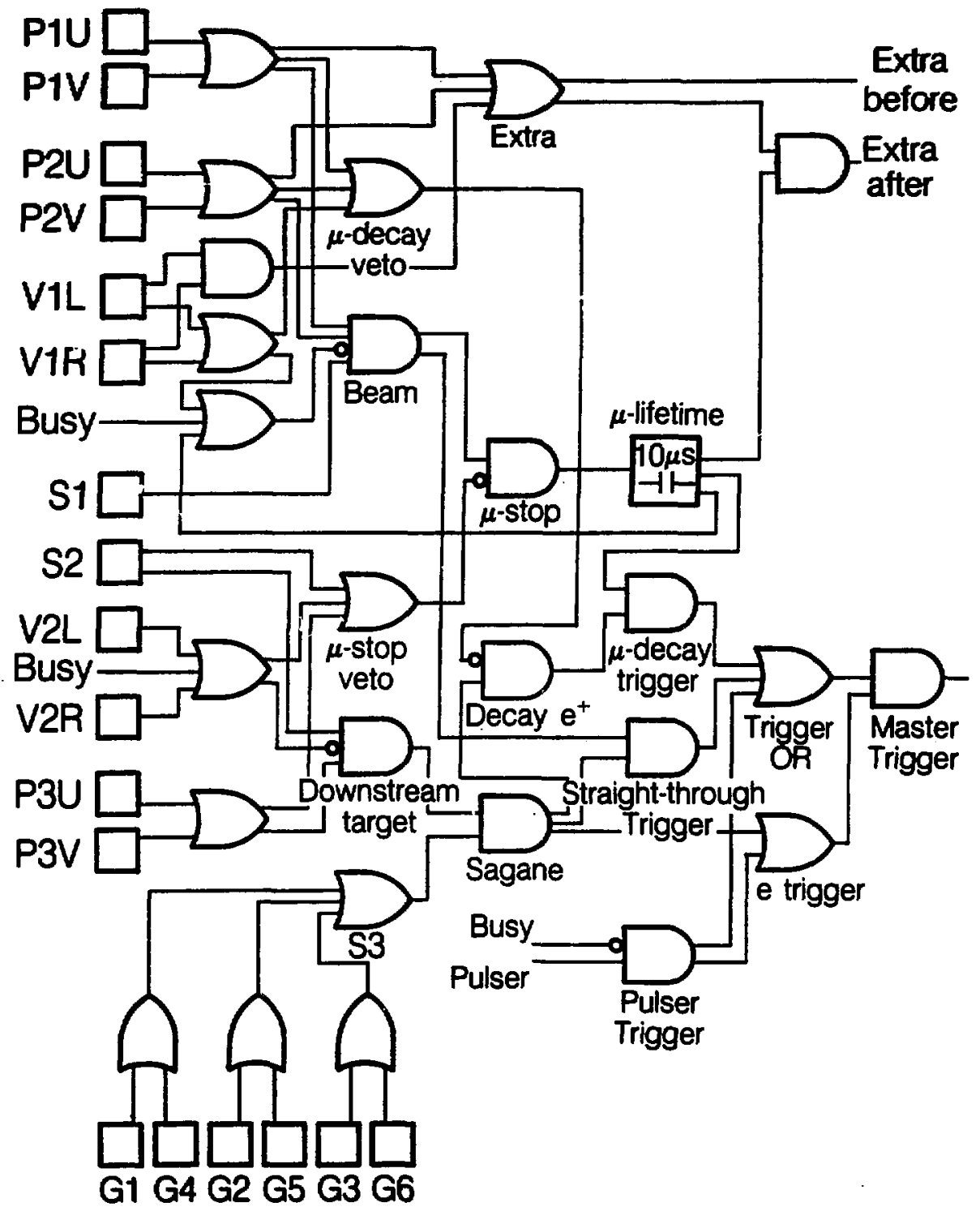

FIGURE (5.6). Essential features of the trigger logic during Run 1. Subsequent ainor changes are described in the text. 
wre-chamber channels.

Beam particles reaching the stopping target region have the signature

$$
\text { Beam }=P 1 . P 2 . S 1 \cdot \overline{V 1}
$$

Particles leaving the stopplng target region and traversing the Sagane spectrometer have the signature

$$
\text { Sagane }=P 3.52 .53 \cdot \bar{V} 2
$$

The straight-through trigger seeks to identify single beam particles which traverse the whole apparatus, and thus requires a coincidence between Beam and Sagane:

$$
\text { Straight-through }=\text { Beam.Sagane }
$$

The $\mu$-decay trigger requires the slgnature of a $\mu$-stop in delayed (0.1-10 us) coincidence with that of a decay $e^{+}$. The $\mu$-stop requirement that the beam particle stops in the stopping target is

$$
\mu-s t c p=\text { Beam. } \overline{\mu-s t o p \text { veto }}
$$

where $\overline{\mu-s t o p \text { veto }}=\overline{\mathrm{P3}} \cdot \overline{\mathrm{S2}} \cdot \overline{\mathrm{v} 2}$

The decay $\mathrm{e}^{+}$requirement that the outgolng downstream particle originates in the stopping target is

$$
\text { Decay } \mathrm{e}^{+}=\text {Sagane. } \overline{\mu-\text { decay reto }}
$$

where $\overline{\mu-d e c a y ~ v e t o ~}=\overline{P_{1}} \cdot \overline{P_{2}} \cdot \overline{V_{1}}$

In Runs 2 and $3 \mathrm{PI}$ and $\mathrm{PZ}$ were removed froc $w$-decay veto and were replaced by the ability to anke software cuts on events with P1 or P2 
siensls near the $\mu$-decay tine. The $\mu$-stop tine was prorided by $S 1$ and the $\mu$-decay time by S2.

in important feature of the logic is the ability to tag, and later reject in sof tware, almost all events where the decay $e^{+}$could have originated from extra $\mu^{+}$rather than the $\mu$-stop muon. This is crucial In the $\mu \mathrm{SR}$ mode of the experiment since extra $\mu^{+}$arriving at random times have correspondingly random precessed spin directions with respect to those of the $\mu$-stop muons. They are therefore equivalent to an admixture of unpolarized muons and thus mimic right-handed current effects. The arrival of each beam particle sets a $10 \mu \mathrm{s}$ latch. If a $\mu$-stop occurs within the $10 \mu \mathrm{s}$ latch the event $1 \mathrm{~s}$ tagged as an 'extra-before'. In addition the arrival times of 'extra-after' beam particles arriving in the $10 \mu$ following the $\mu$-stop were recorded. A high incidence of false extra-after si gnals due to P1 and P2 after-pulsing following the $\mu$-stop were largely ellminated by inserting dead-time notches in 'extra-after'. The resulting 'extra-after-1' and 'extra-after-2' were active from 0.6-10 $\mu$ and 0.85-10 $\mu$ in Run 1, and from $0.3-10 \mu$ and $0.5-10 \mu$ in Runs 2 and 3 respectively. The 1/4 OR of P1 and P2 cathode s1gnals in Extra was replaced In Run 2 by efther a 2/4 or $3 / 4$ coincldence, the cholce depending on the proportional chamber outhode efficiencies. In Run 3 the role of P1 and P2 in Extre was assuned instead by the additional proportional chamber a batween pI and P2. 


\section{4 Data hequisition}

Event data was read fron the CAMAC electronics into a circular buffer of a PDP-11/34 computer using the data acquisition program DA. The data was written to tape after several events were accumulated in the buffer. The program DA also supplied event information to the online analysis program MULTI.

Drift chamber time information was obtained using a LeCroy System 4290. The TDCs were operated in the common-stop mode, with the stop belng provided by the trigger. Digitized time information was transferred to the memory unit which then sent a LAM signal to the PDP-11/34. In addition the $P D P-11 / 34$ read $T D C$ and $A D C$ information from the jicportional chambers and scintillators; TDC information on the $\mu^{+}$ arrival time relative to the cyclotron $r f$ cycle, $\mu^{+}$lifetime, and extra-after times; latches set by proportional chamber wire signals and trigger logic elements; event scalers; and NMR-monitored fields in the beamline dipoles and spectrometer.

The CAMAC electronics were gated-off for $5 \mathrm{~ms}$ (reduced to $200 \mu \mathrm{s}$ during Run 2) while the PDP-11/34 read the event and cleared the CAMAC electronics. In addition a computer 'busy' sl gnal gated-off the trigger logic to prevent another trigger being received until the camac electronics were cleared. It should be noted however that the extra-before latch realned operational during computer 'busy'.

Online infirmation provided by MUTI Included histograns of wire-chaber plane illualnations and autiplicities, the bean spot and angular distributlons, the event time spectrum, scintillator and proportional chamber TDC anc ADC distributions, and the proportion of ewents with extra-before athid extra-after bean particles. 
Typical event rates with 100 wh of protons incident on the production target were $60-70 \mathrm{~Hz}$ in the $\mu \mathrm{SR}$ mode and 25-30 $\mathrm{Hz}$ in the spin-held mode. The $\mu$ SR data presented here were obtained from $1.5 \times 10^{7}$ raw triggers. The cuts described in Chapter 6 retained $5.6 \%$ of the events. 


\section{Chapter 6}

\section{Event Reconstruction}

\section{1 Wire Chamber Alignment}

The relative positions of the wire chamber planes transverse to the beam direction were determined from the mean residuals of reconstructed beam $e^{+}$tracks. Straight track segments were fitted to hits in the horizontal and vertical wire planes of the chamber groups P1, P2, P3, D1 and D2; D3; and D4 [Figure (5.4)] with the solenoid off and no stopping target between P2 and P3. Alignment of wire-chambers P1-D2 as

a single unit ensured that the $\mu^{+}$and $e^{+}$polar angles $\theta_{\mu}$ and $\theta_{e}$ were measured relative to a common axis. The chamber planes were thereby aligned to within $50 \mu \mathrm{m}$, while the $\mathrm{mms}$ residuals were typically $300 \mu \mathrm{m}$ in the drift chambers.

\subsection{Mwon Track Reconsitruction}

Sțraight muon tracks were fitted to hits in proportional chambers P1 and P2. A valid hit was defined to be a signal from at least one, but no more than three, adjacent wires in the same plane. The track was assumed to pass through the center of the hit pattern. One and only one nit was permitted in each plane of P1. One plane of P2 was also required to have one and only one hit, while elther one or two hits were allowed in the other plane. The correct muon track was assuned to be the one agreelng mast closely with the outgolng pasitron track in stopplng target position. Events with reconstructed $\cos \theta_{p}<0.99$ with

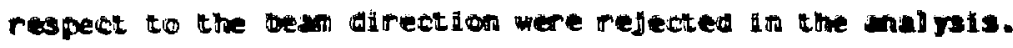




\subsection{Positron Track Reconstruction}

Straight $e^{+}$track segments were fitted separately to hits in the horizontal and vertical projections of the wire-chamber plane groups P3, D1; D2; D3; and D4. Resolution of the left-right ambiguity associated with each drift chamber hit relied on the staggered cells of adjacent sense planes. The first sought track segments of acceptable stralghtness and slope were those with a hit in each of the constituent wire-chamber sense plades. In segments where such tracks were not found the number of sense planes required to have a hit on the track was progressively decreased. If more than one track was found with hits in. the same number of planes the track with the best chi-square was accepted. Tracks in all six segrnents were found in 998 of the triggers.

To guard against fake tracks from spurious hits, cuts were made on the total number of hits in the wire chamber groups. The number of hits in the 10 planes of $\mathrm{P} 3-\mathrm{D} 2$ and $1 \mathrm{y}$ the 10 planes of $\mathrm{D} 4$ were each required to be S18; and in the 12 planes of D3 to be s22. Furthermore, the horizontal and vertical track projections in P3-D2 were each required to have $h 1$ ts in at least 3 of the 5 constituent planes; in $D 3$ to have hits in at least 4 of the 6 planes horizontally and 3 of the 6 planes vertically; and in D4 to have hits in at least 4 of the 6 planes horizontally and 3 of the planes vertically. In addition oniy one nit, as derined in section (6.2), :as pernitted in each plane of P3. The $e^{+}$tracks throuch P3-D2 are not straight because of the longltudinal fleld in the counstrem section of the solenold. The P3-D2 track space polnts were refitted to a curved track based on the first-orcer optles of cylincrically symetric fielde seacoribed in

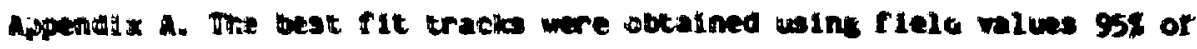


those in Table (5.1).

Appro:imate space: time relations were obtalned by integrating the drift-tiwe distributions of cells almost unifornly illuefrated by decay $e^{+}$in $\mu$ SR runs with the downstream solenold off. The $e^{+}$curved track residuals were used to dynamicaliy fine-tune the space:time relation for each drift chamber plane in each run. The space: time relations for the various planes were stored as arrays of drift distances for each of 512 1-nsec wide drift-time bins. The first 3000 events on each data tape, typically containing $1.2 \times 10^{5}$ events, were used for the fine-tuning after which the tape was rewound and the analysis restarted. If for the $i$ th drift-time bin a residual $r$ was obtained, the drift distances for the $i-8$ to $i+8$ time bins were changed by

$$
\begin{aligned}
& \Delta(i \pm k)=\left(s i g r_{i}\right) r w[9-\max (k, 1)] / 200 \\
& W=\left\{\begin{array}{l}
1.0 \text { if }|r|<0.1 \mathrm{~cm} \\
0.5 \text { if } 0.1 \mathrm{~cm}<|\mathrm{r}|<0.2 \mathrm{~cm} \\
0 \text { if }|\mathrm{r}|>0.2 \mathrm{~cm}
\end{array}\right. \\
& \text { sign }=\left\{\begin{array}{l}
+1 \text { if track coordinate > wire coordinate } \\
-1 \text { if track coordinate < wire coordinate }
\end{array}\right.
\end{aligned}
$$

The changes are therefore largest for the $1^{\prime}$ th and $1 \pm 1^{\prime}$ th drift-time bins and then decrease linearly away from the $1^{\prime}$ th bin. The procedure converges after about 1500 events.

The drift-chamber rms residuals are shown in Table $(6.1)$. The larger rms residual in the Di vertical projection is not well understood. The 37 of events with $e^{+}$tracks in P3-D2 with reduced $x^{2}>20$ were rejected. 


\begin{tabular}{|c|c|}
\hline Delft Chaber & furs Residun (p) \\
\hline Di (horizontal) & 325 \\
\hline (vertical) & 600 \\
\hline D2 & 325 \\
\hline D3 & 250 \\
\hline D4 & 250 \\
\hline
\end{tabular}

Table (6.1)

The $\mathrm{e}^{+}$track segments $f_{i t t e d}$ in $\mathrm{P} 3-\mathrm{D2} ; \mathrm{D} 3$; and $\mathrm{D}_{4}$ were required to satisfy several continuity criteria. First-order optics (Appendix A) extrapolations of the tracks in P3-D2 and D3 into the solenoid bore were required to have both radial agreement, $\Delta R$, and azimuthal agreement, $R \Delta \phi,<2 \mathrm{~cm}$. Extrapolations of the tracks in D3 and D4 into the spectrometer were required to agree to within $4 \mathrm{~cm}$ in both vertical position and impact parameter with respect to the magnet axis, and to agree to within 0.08 in vertical slope. The horizontal position of the $\mathrm{e}^{+}$track determined by the 53 scintillator pair time difference was required to agree w1th the extrapolated D4 track to within $10 \mathrm{~cm}$. Events in which more than one of the three $\$ 3$ scintillator pairs fired were rejected.

Aperture cuts were made in the solenoid and spectrometer. Events with $e^{+}$enitted from the stopping target at radil $>1.8 \mathrm{~cm}$ or with $\cos \theta e^{<0.975}$ were rejected. The $e^{t}$ track radial position at the exit of D2 (aperture radius $8.86 \mathrm{~cm}$ ) was required to be $<8.5 \mathrm{~cm}$. The maximu track radial position in the solenold bore (aperture radius $11.1 \mathrm{~cm}$ ) 
was required to be $<10 \mathrm{~cm}$. The presence of ans lines (Auns 2 and 3) and a heliu bag (Run 2) in addition to D2 signal cables within the solenoid bore made necessary tighter radial cuts or $8.5 \mathrm{~cm}$ in Run 2 and $9.5 \mathrm{~cm}$ In Run 3. The vertical position of the track at the spectrometer exit (vertical aperture $\pm 16.8 \mathrm{~cm}$ ) was required to be within $\pm 15.5 \mathrm{~cm}$ or the median plane. Additional vertical cuts were made at $\pm(6.4-9.4) \mathrm{cm}$ around two horizontal ribs supporting the vacuum window between the spectrometer and D4.

\section{4 Extra Muons}

Most $e^{+}$originating not from the decay of the observed stopped $\mu^{+}$, but from the decay of another $\mu^{+}$were eliminated by rejecting events with 'extra-before' or 'extra-after-1' [section (5.3)] beam particles. The small fraction of events with $\mathrm{e}^{+}$originating from untagged extra $\mu^{+}$ was reduced by requiring continuity between the $e^{+}$and $\mu^{+}$tracks at the stopping target. Requiring track separations $<4.5 \mathrm{~mm}$ rejected $78 \%$ of uncorrelated $\mu^{+}-e^{+}$events and $14 \%$ of correlated $\mu^{+}-e^{+}$events.

Positrons from extra $\mu^{+}$with random arrival times constitute a flat background to the observed $\mu^{+}$decay time spectrum. A comparison of the background levels before and after the cuts described above therefore provides a measure of the efficiency of those cuts. Figure (4.1) shows the Run 2 spin-held data time spectrum after the cuts were made. The fitted background of $1.3 \pm 9.8$ per time bin corresponds to $(3 \pm 22) \times 10^{-5}$ of the time $t=0$ rate. Before making the cuts a spectrum with a similar number of events at early tines had a background of about 1600 per time bin, or $3.6 \times 10^{-2}$ of the $t=0$ rate. 


\title{
6.5 Homentu Meconstruction
}

\author{
The somenta of $e^{+}$pasing through the horizontally focusing \\ cylindrical dipole spectrometer were obtalned to first order from the \\ su of the hor 1 zontal coordinates at the conjugate foci. A nominal $x=1$
} calibration point was provided by the sharp edge at the endpoint of the HSR data. The spectroneter monentur dispersion was measured to be approximately $1.07 \% / \mathrm{cm}$ using $\mathrm{e}^{+}$beams obtalned at several settings or the beamline elements.

Enpirical ad hoc corrections were introduced to make the reconstructed $\mu S R$ data endpoint independent of impact parameter wIth respect to the magnet axis, mean squared (vertical) deviation from the median plane, and vertical position at the spectrometer exit. This procedure was repeated at several spertrometer settings to obtain corrections appropriate for $x \neq 1$ at the standard spectrometer setting. An additional correction eliminated a residual correlation between $\cos \theta_{e}$ and the reconstructed endpoint, which amounted to $\Delta x=0.001$ between the $\cos \theta_{e}=0.975$ and $\cos \theta e^{=1}$ endpoints. The resulting momentum resolution was better than $0.2 \% \mathrm{rms}$.

The spectrometer was re-calibrated with $e^{+}$beams obtained at many beamline settings. In Run 3 two sets of calibration data were taken with the spectrometer at $42 \%, 50 \%, 60 \%, 72 \%, 86 \%$, and $100 \%$ of its standard setting, while in Runs 1 and 2 only the standard setting was used. After allowing for a most probable $e^{+}$energy loss of $1.75 \mathrm{MeV}-\mathrm{cm}^{2} / \mathrm{B}$ in the material upstream of the spectrometer, the $e^{+}$ momentum was assumed to be proportional to the beanline dipole setting9. Any apparent non-1inearities or offsets were attributed to the spectrometer. With the coefficients of the linear and quadratic 


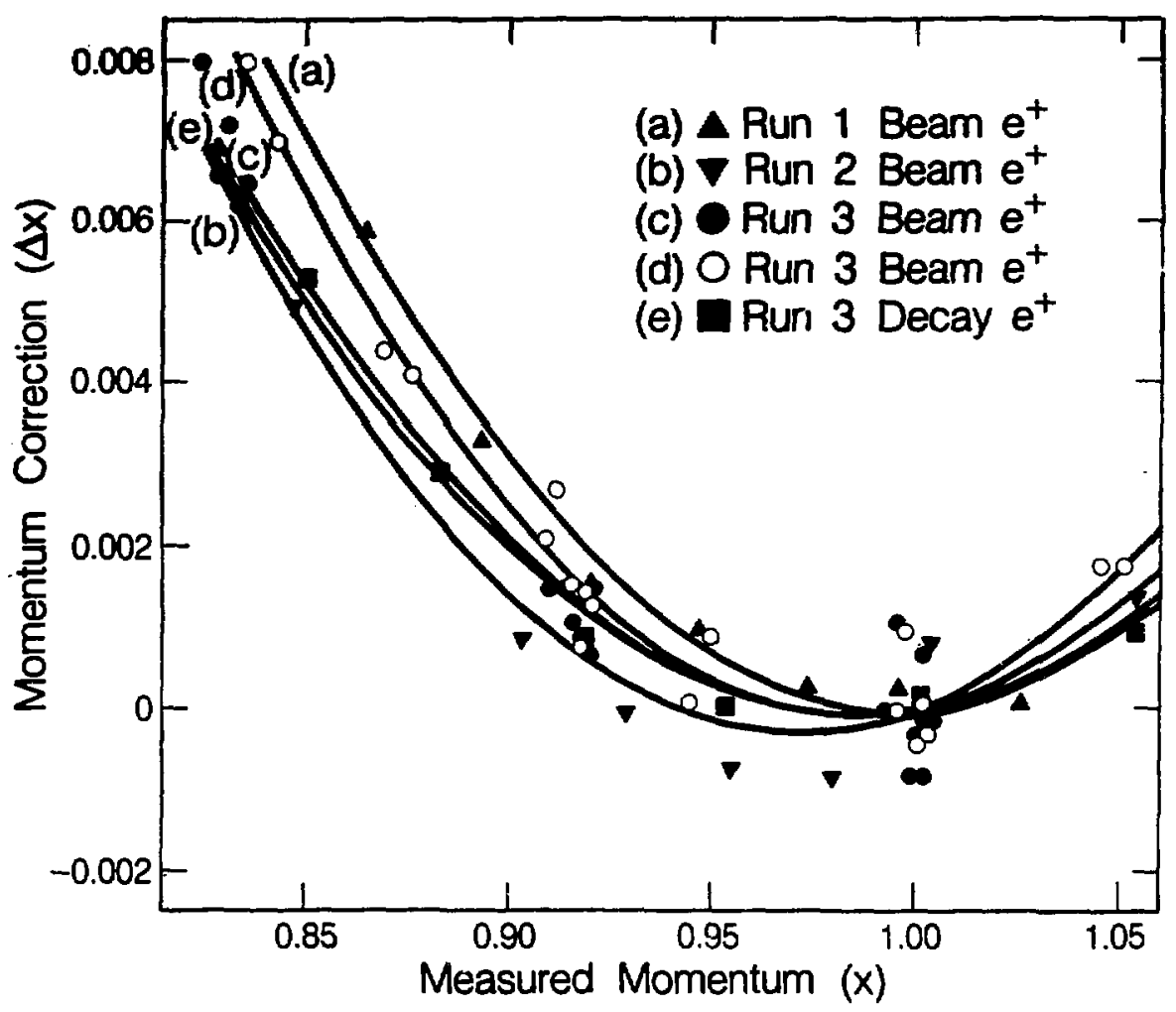

XBL 859-11672

FIGURE (6.1). Mamentum correction versus nominal measured momentum required to yield linear momentum scale with $x=1$ unchanged. The curves are quadratic fits to the polnts. Additional polnts w1th $x>1.05$ in callorations $(c)-(e)$ were included when detergining curves $(c)-(e)$. 
alepersion terms allowed to vary linearly with spectroseter settinc it was found that:

(1) the effective field integral for particles with a $98^{\circ}$ bend angle at the various spectrometer settings increased $(0.22 \pm 0.02)$ I more rapidly than indicated by the NKR probe in the central field region; (11) the IInear dispersion increased by $(1.1 \pm 0.2)$ between the spectrometer $42 \%$ and $100 \%$ settings;

(1ii) the quadratic dispersion was consistent with being constant. An independent calibration, Incorporating the above spectrometer behavior, was performed using the reconstructed $\mu$ SR data endpoints at several spectrometer settings. The result was consistent with the beamline calibrations, thereby indicating that the beamline did not deviate appreciably from the assumed linear behavior.

The calibration data displayed in Figure (6.1) shows the correction required at the standard spectrometer setting to convert the original momentum scale to a linear momentum scale leaving the nominal $x=1$ point unchanged. The mean of the five curves in Figure (6.1) was taken to be. the required momentum correction.

Conversion of the linear monentum scale to an absolute momentum scale is fllustrated by the following exmaple. The endpoint of the Run 3 Al target data was at $x=1.0030$ on the linear momentum scale. Allowing for uniform energy-loss in the material upstream of the spectrcmeter the expected endpoint is at $x=0.9916$ on the absolute nomentu scale. Thus a ractor of C.9886 converts the linear monentum scale to the absolute scale. For data fitting, uniforn energy-loss was added back on to superimpose the data on the energy-loss straggled theoretical spectra (Appendix B). Since the calibration bea $e^{+}$and the decay $e^{+}$ 


\section{traverse sidilar amounts of naterial, the $13 \mathrm{kely}$ error in estimating} the uniform energy-loss has negligible effect on the nonentu ultimately attributed to the decay $\mathrm{e}^{+}$.

The 10 possible systematic error in the momentum calibration was taken to be the standard deviation of the corrections given by the five curves in Figure (6.1). They are shown in Table (6.2) for the centers of the momentum bins used in the data analysis.

\begin{tabular}{ll}
\hline Momentum $x$ & Standard Deviation in Correction $\Delta x$ \\
0.89 & 0.00066 \\
0.91 & 0.00053 \\
0.93 & 0.00040 \\
0.95 & 0.00029 \\
0.97 & 0.00017 \\
0.99 & 0.00006
\end{tabular}

Table $(6.2)$

The above momentum calibration systematic errors are to be added in quadrature with a likely error of \pm 0.0001 in determining the $\mu S R$ data endpoint.

- Events with $x<0.88$ ( $x<0.92$ in Run 1), which have lower statistical power and largen possible systematic errors in momentum reconstruction, were rejected in the analysis. 


\section{Chapter 7}

\section{Data Analysis}

\subsection{Overview}

The $\mu$ SR data in 0.04 us time bins and six 0.02 wide $x$ bins were fitted to

$$
N(t)=N_{0}\left[\int C(x) d x+P_{\mu} A(\tilde{x}) G(t)\langle\cos \theta\rangle_{t} \int D(x) d x\right] \exp \left(-t / \tau_{\mu}\right)
$$

Here $C(x)$ and $D(x)$ are the angle Independent and dependent parts respectively of the radiatively corrected ( $V-A)$ differential decay rate [section (3.3)] smeared by the $e^{+}$energy-loss straggling (Appendix $B$ ) and by a sum of Gaussian momentum resolution functions.

The fit parameters common to all $x$ bins were the $\mu^{+}$mean-life $\tau_{\mu}$, the $\mu^{+}$spin precession frequency $\omega$ and the Initial time $t_{0}$ incorporated Into $\langle\cos \theta\rangle_{t}$, and the two (one) parameters of the Kubo-Tomita (Gaussian) spin relaxation function $G(t)$ [section (4.4)]. The other fit parameter's were the normalizations $N_{0}$ and the asymetries $P_{\mu} A(\tilde{x})$ relative to the $(V-A)$ prediction for each of the six $x$ bins.

Both the spin-held [Figure (4.1)] and $\mu$ SR data [FIgure (4.3)] are consistent with zero background. Since any fitted positive background would increase the apparent decay asymmetry and thus strengthen the limits on right-handed currents, the $\mu S R$ data background was fixed to zero. It was checked that the spin-held data exhibited a consistent exponential decay rate over the tine range used in the $\mu$ SR fits.

The maximum likelihood poisson statistics $\chi^{2}$. derined by

$$
x^{2}-2\left[\left[e_{1}-o_{1}+o_{1} \ln \left(o_{1} / e_{1}\right)\right]\right.
$$


where $o_{1}$ and $e_{1}$ are the observed and expected number or events respectively in the i'th bin, was minimized using a double precision version of the MINUIT minimization program.

7.2 Positron Momentum Spectra

Positrons leaving the stopping target and traversing the other material $\left(=200 \mathrm{mg} / \mathrm{cm}^{2}\right.$ ) upstream of the spectrometer are energy-loss straggled to lower momenta where the unstraggled decay asymmetry is less. The $\mathrm{e}^{+}$energy-loss straggling therefore Increases the apparent asymmetry below the endpoint. Figure (7.1) shows the HSR data momentum spectra for the $A I$ and $A I^{*}$ targets. The greater energy-loss straggling is apparent in the more rounded shoulder in the thicker AI ${ }^{*}$ target data. The radiatively corrected $(V-A) \mu^{+}$differential decay rate [section (3.3)] was evaluated for $\cos \theta=-1,0,1$ at momentum intervals of $\Delta x=0.0004$. These three momentum spectra were energy-loss straggled for both ionization and bremsstrahlung using the formalism of Tsai ${ }^{49}$ ) as described in Appendix $B$.

The three straggled momentum spectra were then smeared by a sum of three Gaussian momentum resolution functions with standard deviations 0,20 , and 30 determined by fitting the time-average $\mu S R$ data to a straggled unpolarized $(\cos \theta=0)$ momentum spectrum.

The integrai of $C(x)$ [equation (7.1)] for each $x$ bin was evaluated by suming the appropriate smeared and straggled decay rate points of the $\cos \theta=0$ spectrua. Similarly the integral of $D(x)$ for each $x$ bin was evaluated by subtracting the sum of the $\cos \theta=-1$ decay rate polnts from the sum of the $\cos \theta-1$ decay rate points and then of riding by 2 . 


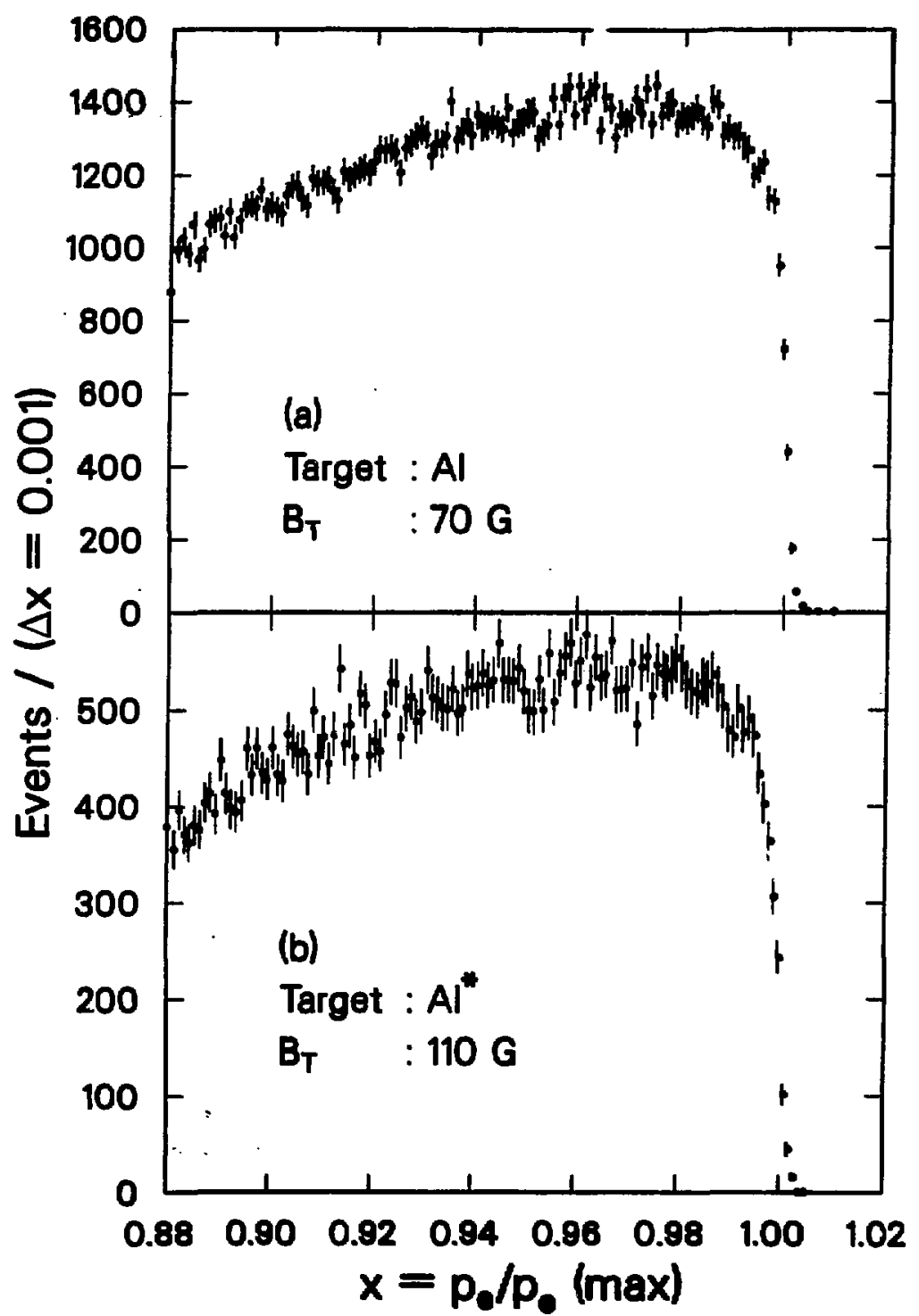

xce ess-mo

FICURE (7.1). Monentu spectra of uSR data with (a) 11 taret and

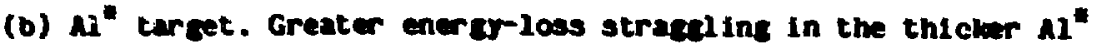
tarcat reaults in a less sharp ede. 


\subsection{The Positron Angular Acceptance}

The angular acceptance of the apparatus for decay $e^{+}$in each $x$ bin is $\mathrm{given}$ by the observed $\hat{\mathrm{p}}_{\mathrm{e}}$ distribution observed in time-average isotropic $\mu S R$ data. In practice one selects a time window which maximizes the number of decay $e^{+}$originating from $\mu^{+}$with precessed spin directions averaging to zero polarization. The $\mu^{+}$polarization directions $\hat{\mathrm{p}}_{\mu}$, assumed to lie along $-\hat{p}_{\mu}$ initially, precess with frequency $\omega=g_{\mu} e B_{T} / 2 m_{\mu} c$. The $\langle\cos \theta\rangle_{t}$ of equation (7.1) is given at any time $t$ by the mean $\cos \theta$ between the $\hat{p}_{e}$ and precessing $\hat{P}_{\mu}$ distributions. If the distributions contain $N$ events

$$
\langle\cos \theta\rangle_{t}=\left(1 / N^{2}\right) \sum_{i j} \sum_{1 j} \cos \theta_{1 j}(t)
$$

where

$$
\begin{aligned}
\cos \theta_{1 j}(t)= & \left(\sin \theta_{\mu} \cos \phi_{\mu}\right)_{i}\left(\sin \theta_{e} \cos \phi_{e}\right)_{j} \\
& +\left[\left(\cos \theta_{\mu}\right)_{i} \sin \omega t+\left(\sin \theta_{\mu} \sin \phi_{\mu}\right)_{1} \cos \omega t\right]\left(\sin \theta_{e} \sin \phi_{e}\right)_{j} \\
& +\left[\left(\cos \theta_{\mu}\right)_{i} \cos \omega t-\left(\sin \theta_{\mu} \sin \phi_{\mu}\right)_{i} \sin \omega t\right]\left(\cos \theta_{e}\right)_{j}
\end{aligned}
$$

Note that if azimuthal symmetry is present equation (7.2) reduces to

$$
\langle\cos \theta\rangle_{t}=\left\langle\cos \theta_{\mu}\right\rangle\left\langle\cos \theta_{e}\right\rangle \cos \omega t
$$

Since the precise precession frequency is unknown until the $f$ it is complete, $\langle\cos \theta\rangle$, is pre-calculated instead for $1^{\circ}$ steps of the precession angle vewt using equation (7.2). As the fit proceeds varlation of the paraneters $\omega$ and $t$. causes the tine bins to correspond to different ranges of the $1^{*}$ precession angle steps. The $\left.\cos \theta\right\rangle_{t}$ for a given time olin is then the mean $(\cos \theta)^{2}$, weighted for ${ }^{*}$ decay within the bin, of the frecession angle steps or fractions thereor

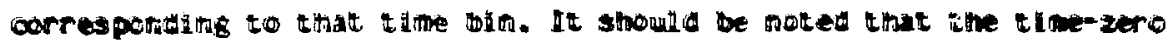


parameter $t_{0}$ is well-defined because the observed $\hat{p}_{\mu}$, and hence $\hat{p}_{\mu}$, distribution defines the time-zero phase of the uSR si mal.

Since the procedure described above is applied to the data in each $f$ it the analysis should be immune to any acceptance changes due, for example, to variations in the $\mu^{+}$beam phase space or detector efficiencies provided the reconstructed quantities for any given event are independent of detector efficiency.

\subsection{Positron Momentum Acceptance}

The $\mathrm{e}^{+}$moment um acceptance is a inaximum near $\mathrm{x}=1$ and decreases to about 605 of maximum at $x=0.88$. Approximating the momentum acceptance changes as linear within each of the $s 1 \times x$ bins a lows simple acceptance corrections to be made.

For each $x$ bin the mean $x$ of time-average $F_{j}=0 \mu$ SR data [section (7.3)] is calculated and compared with the co responding mean $x$ of the theoretical smeared axid straggled unpolarized $(\cos \theta=0)$ momentum spectrum of section $(7.2)$. If the data mean $x$ lies $\left\langle\Delta x_{d}\right\rangle$ from the bin center while the theoretical mean $x$ is at $\left\langle\Delta x_{t}\right\rangle$, the acceptance correction factor multiplying the theoreical spectra $\Delta x$ from the bin center is $f(\Delta x)=1+k \Delta x$ where $k=3 \times 10^{4}\left(\left\langle\Delta x_{d}\right\rangle-\left\langle\Delta x_{t}\right\rangle\right)$. After applying such corrections to each $x$ bin of the smeared and strageled $\cos \theta=-1,0,1$ monentin spectra the integrals of $C(x)$ and $D(x)$ are calculated as described in section (7.2). 
7.5 Monte Car1o Tests

The data fitting method described in the preceding sections was tested using a simple Monte Carlo event generator to produce (V-A) 'events' according to the radiatively corrected decay rate of section (3.3). The fitted as ymmetry normalized to that expected for $(V-A)$ decay, $P_{\mu} A(\tilde{x})$, should be consistent with unity.

Two 'data' sets were generated with different input $\cos \theta_{\mu}, \cos \theta_{e}$, and momentum acceptance distributions. Each 'data' set contained $2.0 \times 10^{6}$ 'events' compared to $0.59 \times 10^{6}$ real events contributing to the final experimental results. The first 'data' set had constant input $\cos \theta_{\mu}(0.99-1.00), \cos \theta_{e}(0.975-1.000)$ and $x(0.88-1.00)$ acceptance distributions, and a $\mu^{+}$spin precession frequency corresponding to $\mathrm{B}_{\mathrm{T}}=70-\mathrm{G}$. For the second 'data' set, generated for $\mathrm{B}_{\mathrm{T}}=110-\mathrm{G}$, the input $\cos \theta_{\mu}$ distribution decreased Iinearly to zero at $\cos \theta_{\mu}=0.99$; the $\cos \theta_{\mathrm{e}}$ distribution decreased 1 inearly by $50 \%$ from $\cos \theta_{e}=1+0.975$; and the $x$ acceptance decreased linearly by $40 \%$ from $x=1 \rightarrow 0.88$. In both cases the input Gaussian spin relaxation function $G(t)$ reduced the $\mu S R$ signal amplitude at $t=10 \mathrm{\mu s}$ to $7 n k$ of its $t=0$ value, whi ah was the largest damping observed in the metal target data. No 'events' were generated for t<0.12 Hs, again imitating the real data. No apparatus effects were Inciuded other than those iaplicit in the input $\cos \theta_{\mu}, \cos \theta_{e}$, and $x$ acceptance distributions. The integrals of $C(x)$ and $D(x)$ in equation (7.1) were therefore deterwined from the momentu spectra of section (3.3) without the energy-loss stragiling and stearing described in $\operatorname{sectin}(\pi \cdot 2)$

The ritted $P_{w} A(\bar{x})$ averiged over $x$ oñ for the two "duth" sets were

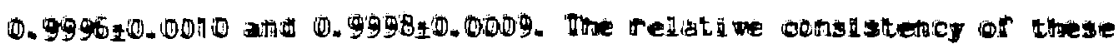




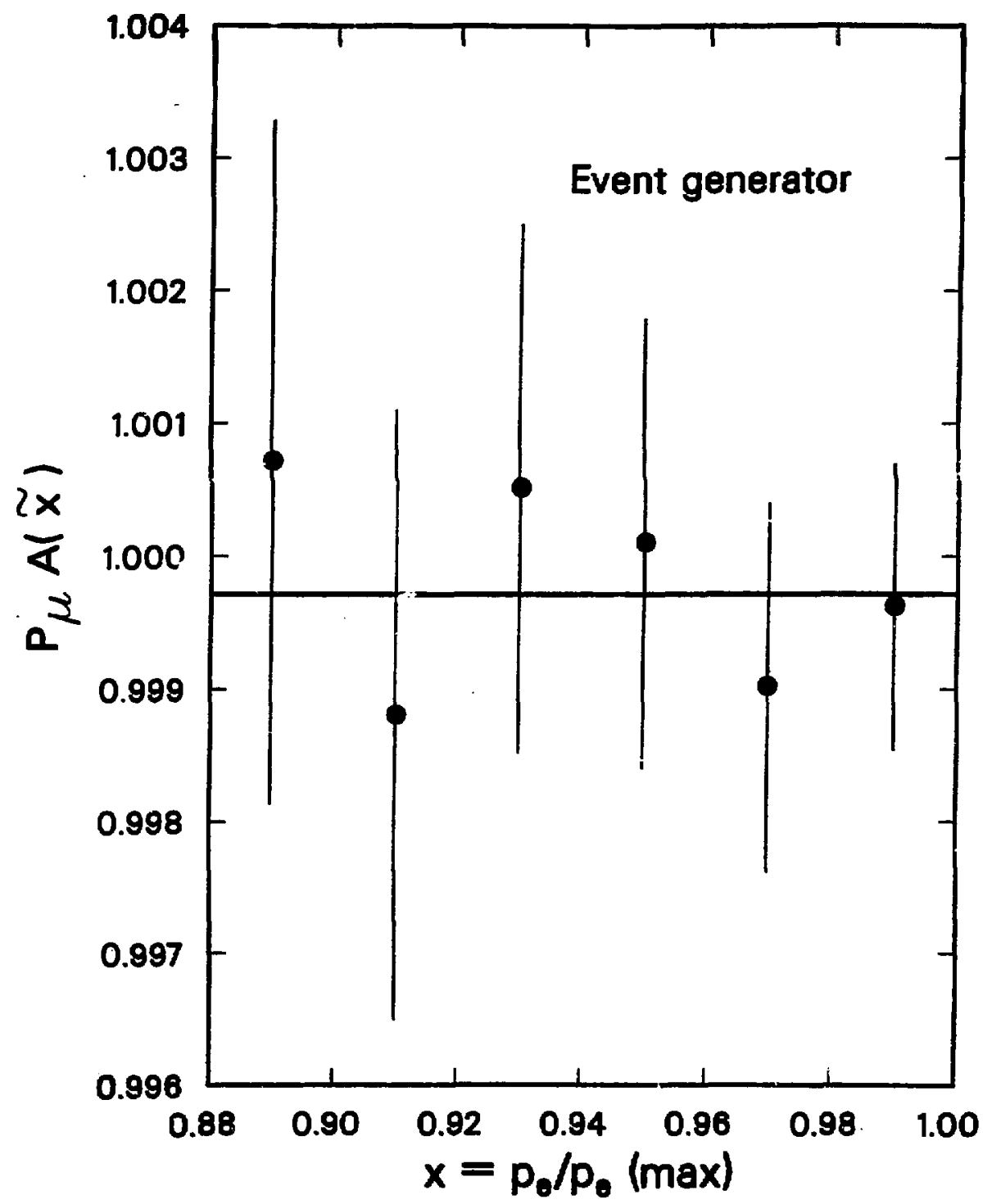

xco $854-174$

FICURE $(7.2)$. Fitted $P_{\mathcal{N}} A(\bar{x})$ for $4.0 \times 10^{6}$ Monte Carlo events senerated with $P_{\mu} A(\bar{x})=1$. The weightec aean fitted $P_{\mu} A(\bar{x})=0.9997 \pm 0.0007$. 
ralues, and of the comblned value $0.9997 \pm 0.0007$ with the input $P_{\mu} A(\tilde{x})$ of unity at a statistical level 6.7 tioes that of the real data gives confidence in the ritting procedure. The comblned fitted $P_{p} A(\tilde{x})$ for each $x$ bin are plotted in Figure $(7.2 j$.

\subsection{Data Fitting Results}

The results of the various fits described in this section are tabulated in Tables (C.1) and (C.2) of Appendix C. All runs except those with some known deficiency were included in the fics. For Example, several runs were rejected because of partial deflation of the helium bag (present only in Run 2) between drift-chambers D2 and D3.

The final results are based on the normalized asymmetries $P_{\mu} A(\tilde{x})$ fitted to each $x$ bin for the various stopping targets and $B_{T}$ settings. The results of these fits are shown in Table (c.1) for both Gaussian and Kubo-Tomita $\mu^{+}$spin relaxation functions $G(t)$. The $f$ itted initial depolarization $(12.4 \pm 0.9 \%)$ in 11 quid He may be due to $\mu^{+}-e^{-}$spin exchange processes during or shortly after $\mu^{+}$thermalization. The fitted $P_{\mu} A(\tilde{x})$ averaged over $x$ bins for each metal target data set are displayed in Figure (7.3). The Run $2 \mathrm{Cu}$ and $\mathrm{Cu}^{*}$ target data exhibits significantly smaller $P_{\mu} A(\tilde{x})$ [4.80 for Gaussian $\left.G(t)\right]$ than the other metal target data. Muon range-straggling calculations [Table (5.2)] show that the $156 \mathrm{mg} / \mathrm{cm}^{2} \mathrm{Cu}$ target was too thin to stop the म $^{+}$well within the target, while the $222 \mathrm{mg} / \mathrm{cm}^{2} \mathrm{Cu}^{*}$ target, composed of two foils, may have suffered from $\mu^{+}$stopping between the folls.

The $P_{\mu} A(\tilde{x})$ for all $\times$ blns and targets should be consistent if the momentum calibration is correc, If the decay parameters $\rho$ and $\delta$ have 


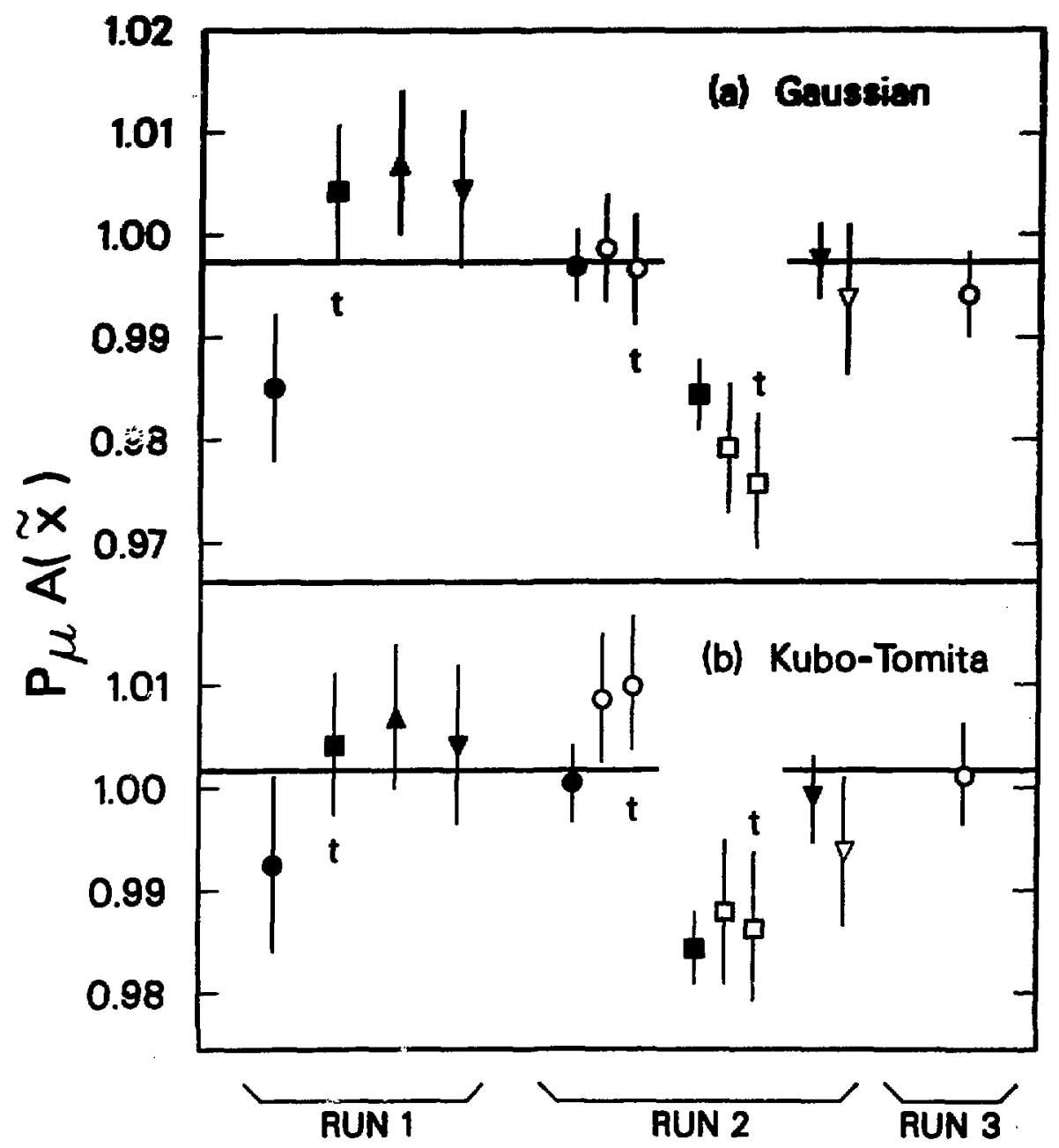

xce 089-108

FICURE (7.3). Weighted mean fitted $P_{\mu} A(\bar{x})$ for the metal target data sets with (a) Gaussian and (b) Kubo-Tomita $\mu^{+}$spin relaxation forms. Targets are $A l$ (circles), Cu (squares), $A_{B}$ (triangles) and Au (Inverted triangles). Thick targets $\mathrm{Al}^{*}$ and $\mathrm{Cu}$ (are marked "t". Spin precessing flelds are $B_{T}=70-G$ (closed symbols) and $B_{T}=110-G$ (open symbols). 
their $\left(V_{t}\right)$ values [see equation (3.3)], and if the tarets do not produce differing initial $w^{*}$ depolarizations. Excluding the He and fiun $2 \mathrm{Cu}$ and $\mathrm{Cu}^{*}$ data, the resalning $52 \mathrm{P}_{\mu} \mathrm{A}(\overline{\mathrm{x}})$ values for Gaussion $\mathrm{G}(\mathrm{t})$ have a mean of $0.9973 \pm 0.0016$ wtth $x_{32}^{2}=63.5$ (C.L. -11 I). Incluston of Run $2 C u$ and $C u^{*}$ ylelds a mean $P_{\mu} A(\bar{x})=0.9934 \pm 0.0014$ with $x_{6,-106.7}^{2}$ (C.L. -0.27 ). The final result is based on the metal target data sets excluding Run $2 \mathrm{Cu}$ and $\mathrm{Cu}^{*}$. The Run $1 \mathrm{Cu}^{*}$ data se: was retalned because there the $\mu^{+}$stopped 0.5 ras straggling lengths deeper in the second foll due to the proportional chamber gas being methane/methylal instead of magic gas. The $x$ bin averaged $P_{\mu} A(\tilde{x})$ in Figure (7.3) for the ten remaining data sets are statistically consistent with $x_{9}^{2}=8.4$ (C.L. -498$)$. Figure $(7.4)$ shows the $P_{\mu} A(\tilde{x})$, averaged over the remaining metal targets, for each $\mathrm{x}$ bin with the 10 possible monentum calibration systemat Ic error added in quadrature to the statistical error. With only the statistical errors the points have $x_{s}^{2}=7.5$ (C.L. $=19 \%$ ). The line is the best fit using the world average $\delta$ and $\rho$ values [section (9.4)]. Table (C.1) shows that for Run $1 \mathrm{Ag}, \mathrm{Au}$, and $\mathrm{Cu}^{*}$, and for Run $2 \mathrm{Au}$ $(70-G$ and $110-G)$ the Kubo-Tomita $G(t)$ fits did not have $x^{2}$ less than the Gaussian $G(t)$ fits. Since for these data sets the Kubo-Tomita $G(t)$ closely approeches its Gaussian limit the true $P_{\mu} A(\tilde{x})$ may be less than that obtained with Gaussian $G(t)$. Refitting with a form $G(t)=\exp \left(-\alpha t^{\beta}\right)$ yielded $\beta>2$, lower $\chi^{2}$, and lower $P_{\mu} A(\bar{x})$ for $R u n 1 A g, A u$, and $C u^{*}$ but not for Run 2 Au. For the 10 metal targets and Kubo-Tomita $G(t)$ the mean $P_{\mu} A(\bar{x})=1.0020 \pm 0.0018$. When the lower values for Run $1 \mathrm{Ag}, A u$, and $C u^{*}$ are used instead the mean $P_{\mu} A(\bar{x})-1.0013 \pm 0.0018$, which is still significantly larger than the Gaussian $G(t)$ mean $P_{\mu} A(\bar{x})=0.9973 \pm 0.0016$. Thus the global use of Gaussian $G(t)$ appears to have provided a lower 


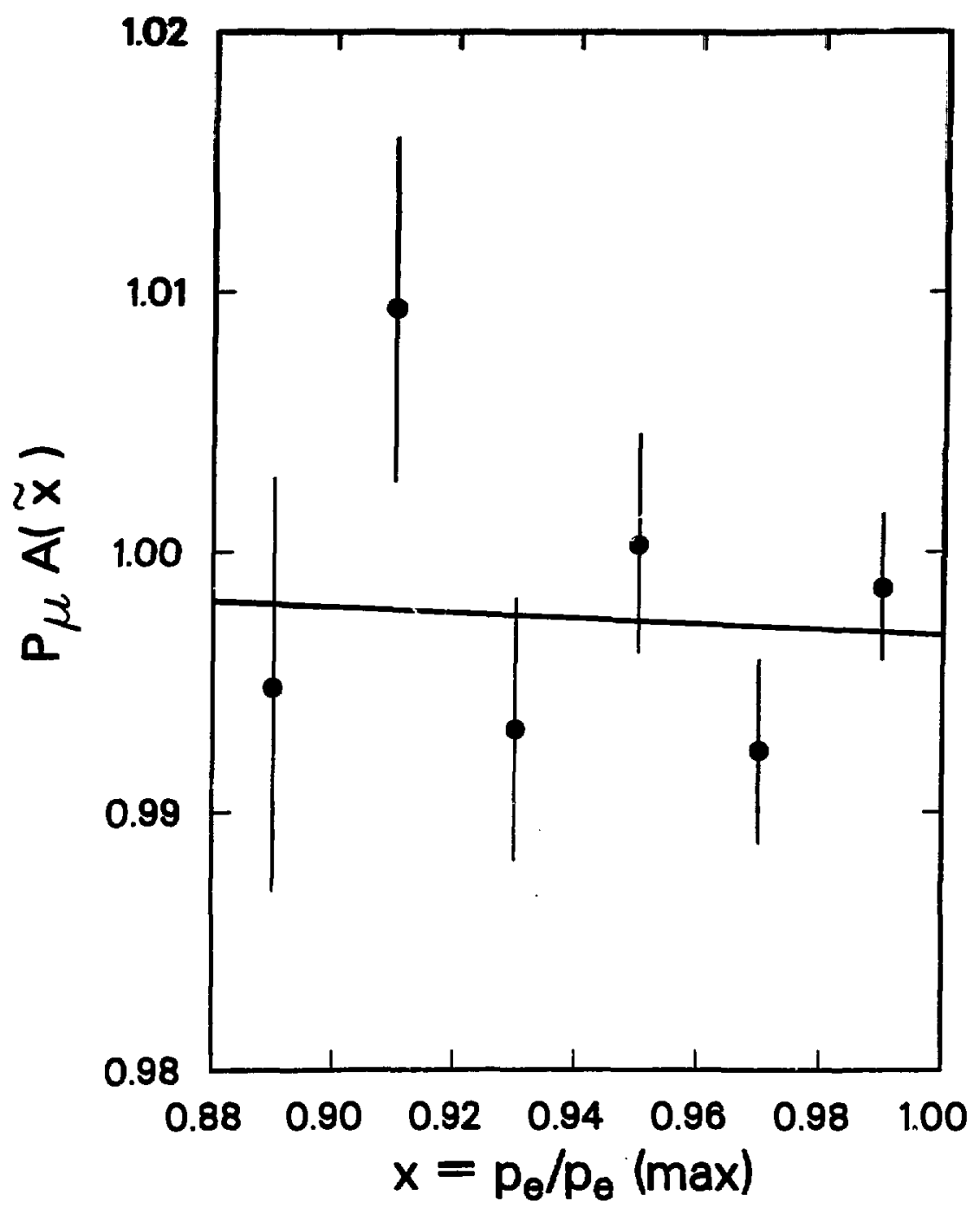

XC0 863-168

FIGURE (7.4). Weighted mean fitteo $P_{\mu} A(\tilde{x})$ in each $x$ bin for metal targets excluding fun $2 \mathrm{Cu}$ and $\mathrm{Cu}^{*}$. Exror bars are statistical errors added in quadrature to the possible momentum calibration systematic error. The fitted line assumes the Horld average values of $\delta$ and $p$. 
bound on $P_{\mu} A(\bar{x})$.

Three auxiliary fits were ande to each data set. Firstiy, with $G(t)$ fIxed to unity a common $P_{\mu} M(\bar{x}) G(t)$ was fitted to the $x$ bins for each $\Psi^{*}$ spin precession period. The fitted $P_{\mu} A(\bar{x}) G(t)$ tabulated in Table $(C .1)$ and plotted in Figure (7.5) versus the tiwe-range midpoint indicate the actual form of $\mathrm{G}(\mathrm{t})$. The curves in Flgure (7.5) correspond to the Gaussian $G(t)$ obtained in the primary fits. The aluminum target data, whlch has a significantly better $x^{2}$ for Kubo-Tonita $G(t)$ is seen to exhibit an actual $G(t)$ far closer to Gaussian than exponential. The $\mu S R$ signal damping in Al is much larger than observed in other experiments, and may be due to $\mu^{+}$trapping in cracks or other defects in the cold-rolled Al foils.

Secondly, for each data set a common $P_{\mu} A(\bar{x})$ was fitted to the $x$ bins for each of five 0.005 wide $\cos \theta_{e}$ bins with a Gaussian $G(t)$ fixed to that obtained in the primary fit. The results are shown in Table (C.1). The 50 measurements in the data sets contributing to the final results have $x_{49}^{2}=52.4\left(C, L_{0}=33 \%\right)$. The combined data in Figure (7.6) are consistent $\left(x_{4}^{2}=1.4, C . L . * 85 \%\right)$ with fitted $P_{\mu} A(\bar{x})$ independent of reconstructed $\cos \theta_{\mathrm{e}}$.

Thirdly, a common $P_{\mu} A(\tilde{x})$ was fittej to the $x$ bins for individual runs with the Gaussian $G(t)$ obtained in the primary fit for the corresponding data set. The results are tabulated in Table (C.2). Figure (7.7) displays the results as a histogram of the deviation of the individual run $P_{\mu} A(\bar{z})$ from the data set nean in units of the individual run statistical error. The histogram is consistent $\left(x_{:=11}^{2}=11,6\right.$. C. $\left._{0}=608\right)$ with a normal distribution truncated at \pm 40 . There is no evidence for 'bad' runs apart from those rejected for known 


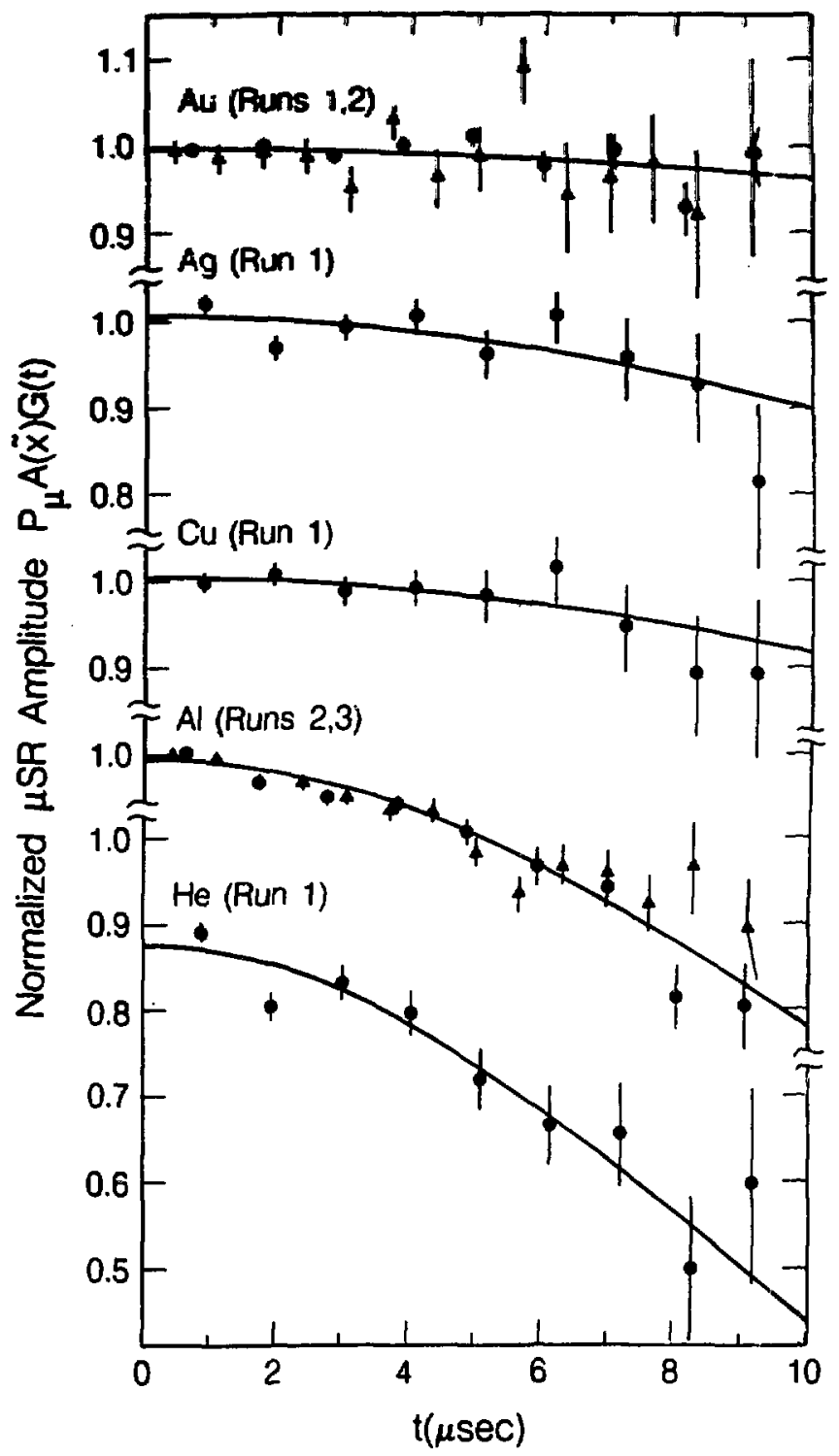

XBL $8412-6007$

FIGURE (7.5). Values of $P_{\mu} A(\bar{x}) G(t)$ for each $\mu^{+}$spin precession cycle with $B_{T}=70-G$ (circles) and $B_{T}=110-G$ (triangles). The curve3 assume Gaussian $p^{+}$spin relaxation functions $G(t)$. 


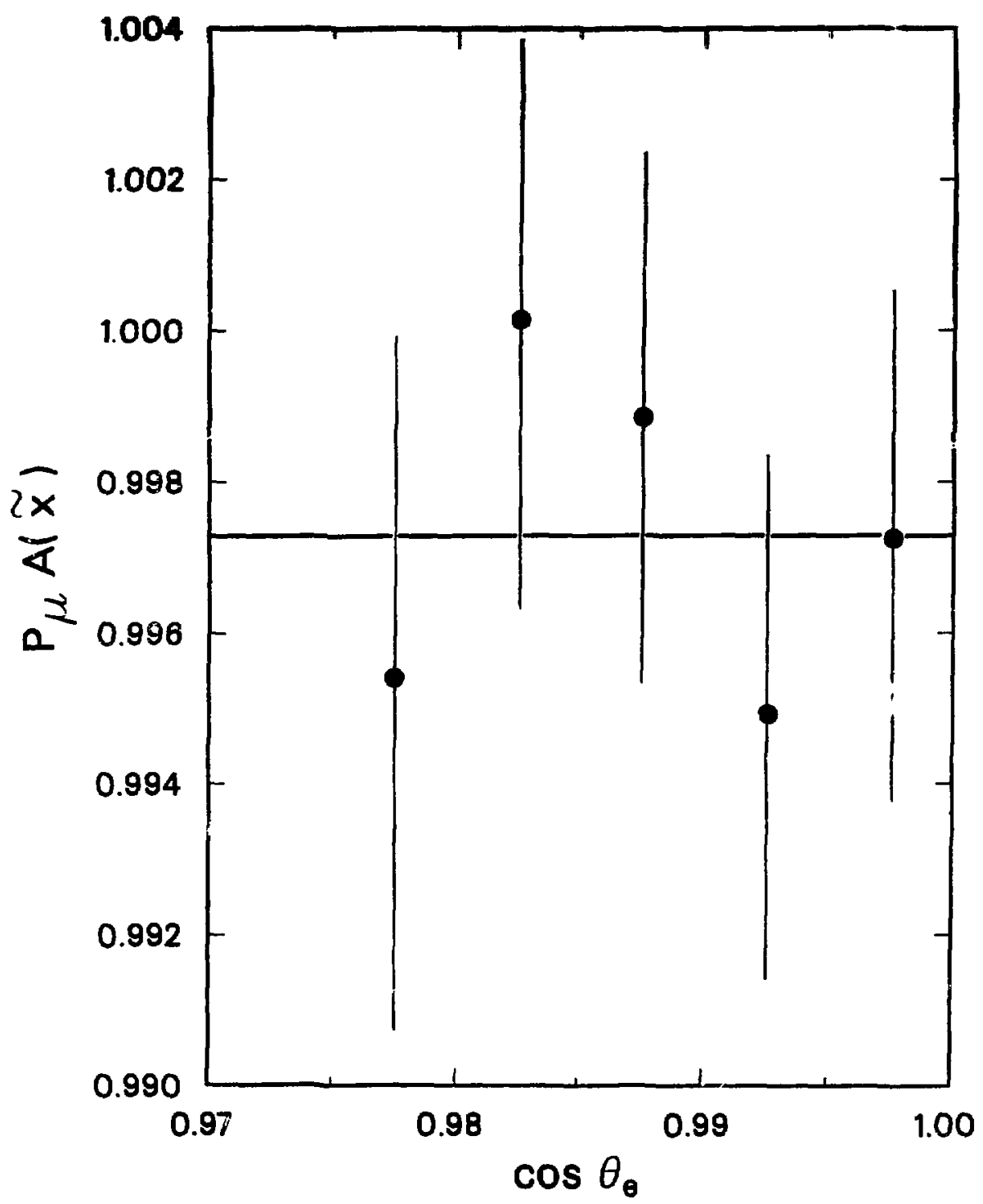

XCG 854-175

FIGURE (7.6). Weighted mean fitted $P_{\mu} A(\bar{x})$ in each $\cos \theta_{e}$ bin for the metal targets exclucing Run $2 \mathrm{Cu}$ and $\mathrm{Cu}^{*}$. 


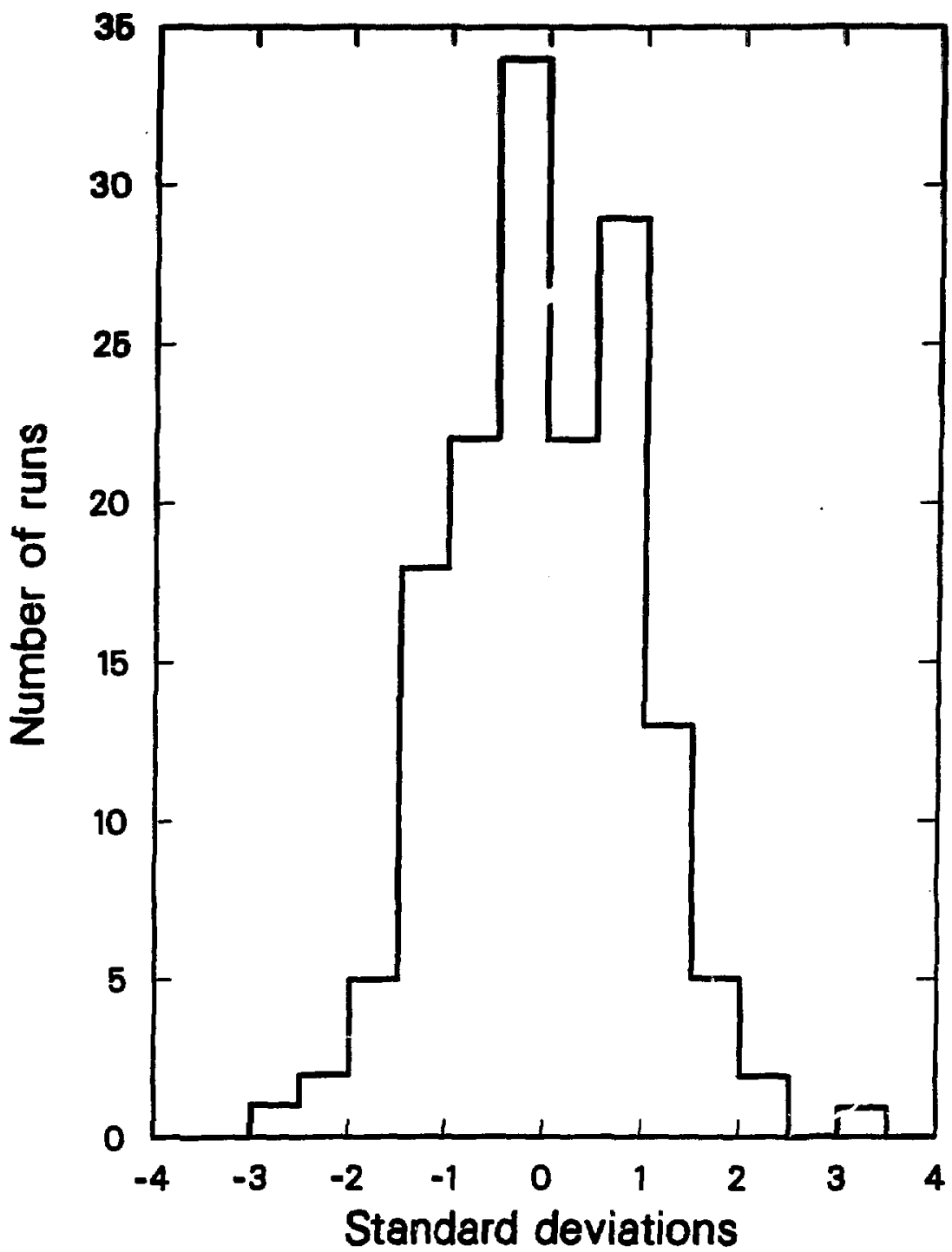

XCG 863-i:7

FIGURE (7.7). Histogram of the deviation of the individual run $P_{\mu} A(\bar{x})$ from the corresponding data set mean in units of the Individual run stailstical error. All runs listed in Table (C.2) are Incluted. 
Eu

deficiencies prior to data fitting. The $\left.P_{\mu} \mathbf{M} \bar{x}\right)$ of individual runs contributing to the final results are displayed in the figure (7.8) histogram.

In each of the three auxiliary fits the $\mu^{+}$spin precession frequency, the initial time $t_{0}$ and the muon mean-life were fixed to the corresponding values determined in the primary fits. The statistical errors on $P_{\mu} A(\tilde{x})$ in the auxiliary fits have been increased by the $5 \%$ required to compensate for the fixed parameters. 


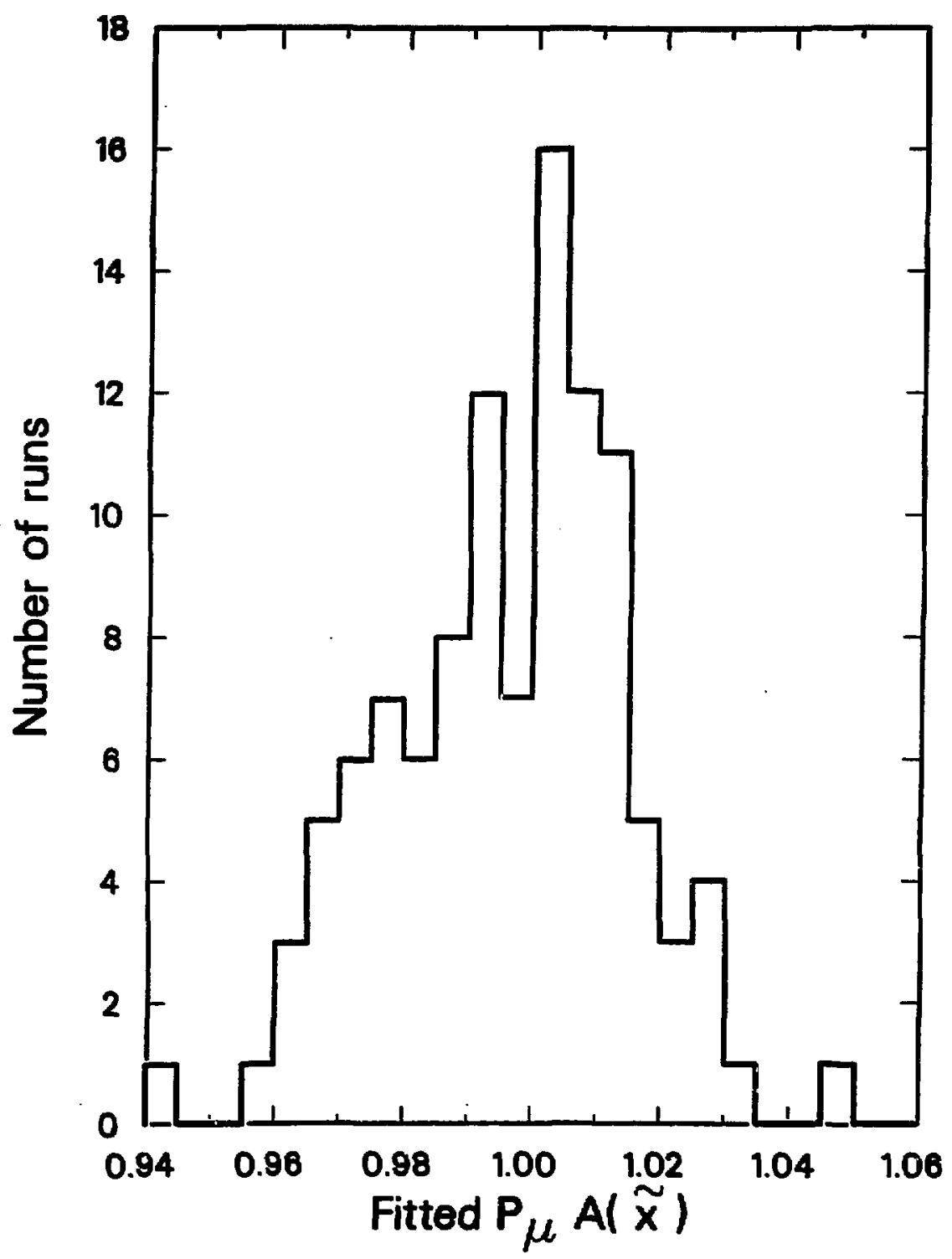

XCO 064-170

FiGJRE (7.8). Histogran of the Indiyldual run $P_{\mu} \Lambda(\bar{x})$ for the retal targets excluding Run $\mathrm{Z} \mathrm{Cu}$ and $\mathrm{Cu}^{*}$. 


\section{Chapter 8}

\section{Corrections and Systematics}

\subsection{Corrections}

8.1.1 Muon Depolarization in Scattering with Electrons

The muon beam polarization is reduced by spin exchange effects in scattering with the unpolarized electrons of the medium ${ }^{30}$ ). Assuning that the muon energy-loss for $E>3 \mathrm{keV}$ is due entirely to scattering with electrons, the calculation in section (4.2) shows the polarization of the stopped beam is 0.9993 of the initial $P_{\mu}$. A possible error of \pm 0.0002 is assigned to this estimated depolarization. The fitted values of $P_{\mu} A(\tilde{x})$ should therefore be corrected upwards by a factor of $1.0007 \pm 0.0002$.

\subsubsection{Coulomb Scattering}

The method for obtalning the $\langle\cos \theta\rangle_{t}$ for each time bin was discussed in section (7.3). It was shown that if azimuthal symmetry applied

$$
\langle\cos \theta\rangle_{t}=\left\langle\cos \theta_{\mu}\right\rangle\left\langle\cos \theta_{e}\right\rangle \cos \omega t
$$

Coulomb scattering is relativistically helicity conserving and non-relativisticaliy spin conserving. The non-relativistic limit is assimed to apply to the $\mu^{+}$, which initially have $8=0.27$. The effect of multiple couronb scattering is to misall directions, and to misalign the true and measured $e^{*}$ emission 
directions. Consequently corrections must be made to both $\left\langle\cos \theta_{\mu}\right\rangle$ and $\left\langle\cos \theta_{\mathrm{e}}\right\rangle$

To a good.approximation material upstream of the midpoint between proportional chambers $\mathrm{P} 1$ and $\mathrm{P} 2$, which measure the incoming muon direction, contributes to the misalignment of the $\mu^{+}$spin and momentur directions while material downstream of this point does not. However, scattering in the production target material and in the material near P1 require corrections of opposite $\operatorname{sign}$ to $\left\langle\cos \theta_{\mu}\right\rangle$. Consider an idealized beamline which admits only $\mu^{+}$with momenta along the beam axis after Coulomb scattering in the production target. Suppose the amount of material near $P 1$ is negligibly small. Since the $\mu^{+}$spins and momenta are misaligned $\left|\left\langle\cos \theta_{\mu, \operatorname{spin}}\right\rangle\right|\left\langle\left|\left\langle\cos \theta_{\mu}\right\rangle\right|=1\right.$. Now suppose the amount of production target materlal is negligibly small so that the $\mu^{+}$ leave the beamline with spins and momenta aligned along the beam axis. Scattering near P1 leaves the spins aligned along the beam axis and now $\left|\left\langle\cos \theta_{\mu}\right\rangle\right|<\left|\left\langle\cos \theta_{\mu, \text { spin }}\right\rangle\right|=1$.

The mean production target thickness traversed by the $\mu^{+}$was $6.2 \mathrm{mg} / \mathrm{cm}^{2}$. The thickness of the other material upstream of the midpoint of $\mathrm{P1}$ and P2 was $18.4 \mathrm{mg} / \mathrm{cm}^{2}$. Scattering near P1 should therefore dominate, requiring a net upwards correction to $\left\langle\cos \theta_{\mu}\right\rangle$ and a downwards correction to $P_{\mu} \mathcal{\Lambda}(\tilde{x})$. It should be noted that acceptance effects and software cuts preferentially reject potentlal events with the largest $\mu^{+}$scattering angles near P1. Detailed Monte Carlo studien using calculations of itoilere scatte ing 30,31 ) yield a correction for $\left\langle\cos \theta_{y}\right\rangle$ of +0.0003 , and hence a cor vestion facter for $P_{\mu} A(\bar{x})$ of 0.9937 . A possible error of \pm 0.0002 is assigred to the correction. The $e^{*}$ scattering is more transparent. Events in which the $e^{*}$ is 
scattered out of the angular acceptance, i.e. to $\cos \theta_{e}<0.975$, are lost while events in which the $e^{+}$are scattered into the angular acceptance are gained. Thus $\left\langle\cos \theta_{e}\right.$, true $\rangle\left\langle\left\langle\cos \theta_{e}\right\rangle\right.$ and an upwards correction to $P_{\mu} A(\tilde{x})$ is required. Monte carlo studies yield a correction factor, averaged over the various stopping targets, for $P_{\mu} A(\tilde{x})$ of 1.0002. A possible error of \pm 0.0001 is assigned to this correction.

\subsubsection{Extra Muons}

The number $\mathrm{N}$ of muons expected to be present in the stopping target 1s determined by the $\mu^{+}$beam rate $\lambda$ and mean-1ife $\tau_{\mu}$ :

$$
\frac{d N}{d t}=\lambda-\frac{N}{\tau_{j}}
$$

If the beam is turned on at $t=0$

$$
N(t)=\lambda \tau_{\mu}\left[1-\exp \left(-t / \tau_{\mu}\right)\right]
$$

Assuminz an average proton current of $80 \mu \mathrm{A}$ incident on the production target the $\mu^{+}$beam rate is estimated to be $\lambda=1.5 \times 10^{4} \mathrm{~Hz}$ from the observed $\mu$-stop rate corrected for dead-time.

Events with extra $\mu^{+}$arriving up to $10 \mu \mathrm{s}$ before the $\mu$-stop are tagged as 'extra-befores' and are rejected. The residual admixture of extra-befores arriving before the 10 us rejection period is therefore $\lambda \tau_{\mu} \exp \left(-10 \mu S / \tau_{\mu}\right)=3.5 \times 10^{-4}$. The requirement of continuity between the $\mu^{+}$ and $e^{*}$ tracks at the stoppling target [section $(6.4)$ ] is estimated to reduce the admixture to $0.9 \times 10^{-1}$. Taking these extra-before $w^{*}$ to be time-average unpolarlzed wi th respect. :0 the y-stop muss lonplies a correction actor of 1.000 for the fisted $B_{m} A(\bar{x})$. A possible error of 


\section{t0.0001 is assigned to this correction.}

A sidiar calculation for extra-after ${p^{+}}^{+}$arribing mobserved durlins the 0-0.3 us notch (Runs 2,3) in extra-after-1 [section (5.3)] 1apifes correction factors of 1.0005 for $B_{T}=70-G$ and 1.0011 for $B_{T}=110-G$. However, the after-pulsing in $\mathrm{Pl}$ and $\mathrm{P} 2$ whlch necessitated the notch cause some extra-after $\mu^{+}$arriving within the notch to be observed as after-pulses after the notch. The above corrections are therefore too large. If extra-after-2, with a 0-0.5 $\mu$ s notch (Runs 2, 3), is used instead of extra-after-1 the mean fitted $P_{\mu} A(\bar{x})$ is reduced by 0.0009 whereas the calculated reduction is 0.0013 . Thus $30 \%$ of the effect appears to be lost to after-pulsing. A larger proportion of extra-after $\mu^{+}$arriving within the shorter $0-0.3 \mu$ sotch should be observed as after-pulses. It is estimated that the calculated corrections should be reduced by $50 \%$. Averaging over the two $\mathrm{B}_{\mathrm{T}}$ values and including the effect of the longer $0.6 \mu \mathrm{s}$ notch in Run 1 yields a correction factor of 1.0004 for the fitted $P_{\mu} A(\tilde{x})$. A possible error of \pm 0.0003 is conservatively assigned to this correction.

\section{8.i. 4 Cloud Muons}

Figure (5.3) indicates that $98 \%$ of cloud $\mu^{+}$are eliminated by the $r f$ time cuts. The fitted asymetry is reduced by 0.015 when no $r f$ time cuts are made. The residual $2 \%$ of cloud $\mu^{+}$therefore require an estimated correction factor of $1.0003 \pm 0.0002$ for the fitted $P_{\mu} A(\bar{x})$. 


\subsubsection{Longitudinal Field Component}

Any residual longitudinal component in the $\mu^{+}$spin precessing field reduces the apparent $\mu S R$ signal amplitude.

The methods used to null the $=40-G$ longitudinal field in the stopping target region [section $(5.2 .1)]$ are estimated to leave an rms residual longitudinal field $=1-G$.

In addition the $\mu^{+}$experience the longitudinal components of the random local fields due to the nuclear magnetic dipoles. As noted in section (4.4) the local fields are a few Gauss for aluminum and copper. However, at room temperature the $\mu^{+}$are mobile and sample many different local flelda in succession. The time-average local field seen by the $\mu^{+}$is therefore reduced. Assuming a unfform applied transverse fleld, the local field $\Delta B$ is related to the static I Inewidth o by equation $(4.3)$ : $\left\langle\Delta B^{2}\right\rangle=20^{2} / \gamma_{\mu}{ }^{2}$. Taking $o^{2}$ from flts using the Gaussian spin relaxation function $G(t)=\exp \left(-\sigma^{2} t^{2}\right)$ ylelds effective rms local fields $\Delta B_{\text {rms }}$ ranging from $0.2-G$ for the Au target to $1.0-G$ for the $A l$ target. The rms longitudinal local field component is $\Delta B_{\mathrm{rms}} / \sqrt{ } 3$.

After adding in quadrature to obtain the total longitudinal fleld $B_{\ell}$, the correction factor for $P_{\mathcal{L}} A(\tilde{x})$ is $1 / \cos \left(B_{\ell} / B_{T}\right)=1.0001$ when averaged over the $B_{T}$ values. A possible error of \pm 0.0001 is assigned to this correction.

\subsubsection{Tulins Brrors}

Any randoen spreads in the times attributed to the $\mu$-atop and m-deany rellatlwe to the true tines effectively smenr the ush aighal, thereby reducithe its apparent aplitube. The time apread of algais 
from the left and right photomutlipliers viewing $S 1$ and $S 2$ with respect to the mixed $S 1$ and mixed S2 signals allow an estimate of 2 ns for the rms error on the lifetime of the individual muons. The $\mu^{+} \operatorname{spin}$ precession period is $T=1.06 \mu$ for $B_{T}=70-G$ and $T=0.65$ Hs for $B_{T}=110-G$, resulting in a correction factor for $P_{\mu} A(\bar{x})$ of $1 / \cos (2 \pi \times 2 n s / T)=1.0001$ when averaged over $\mathrm{B}_{\mathrm{T}}$ values. A possible error of \pm 0.0001 is assigned to this correction.

\subsubsection{Summary}

The corrections discussed in the preceding sections are summarized in Table $(8.1)$. The combined correction factor $1: 1.0016 \pm 0.0006$. The possible errors in the $\mu^{+}$and $e^{+}$Coulomb scattering corrections have been added linearly, as have the possible errors i: the extra-before and extra-after muon corrections, before being added in quadrature to the other possible errors. 
Muon depolarization in scattering with $\mathrm{e}^{-}$ $1.0007 \pm 0.0002$

Coulomb scattering of muons

$0.9997 \pm 0.0002$

Coulomb scattering of positrons

$1.0002 \pm 0.0001$

Extra-before muons

$1.0001 \pm 0.0001$

Extra-after muons

$1.0004 \pm 0.0003$

Residual cloud muons

$1.0003 \pm 0.0002$

Longitudinal field component

$1.0001 \pm 0.0001$

Timing errors

$1.0001 \pm 0.0001$

Total correction factor

$1.0016 \pm 0.0006$

Table (8.1) 


\subsection{Systematic Errors}

The major sources of possibie systematic error, other than those assoclated with the corrections of section (8.1), are discussed in the following sections. Other possible systematic errors are estimated to be small compared to \pm 0.0001 .

\subsubsection{Reconstruction of $\theta_{\mu}$ and $\theta_{e}$}

The main sources of possible systematic error in the reconstruction of $\cos \theta_{\mu}$ and $\cos \theta_{e}$ are longitudinal misalignment of the wire-chambers and the approximations involved in using the first-order optics formalism (Appendix A) to determine the $e^{+}$track.

A possible error of $\pm 2 \mathrm{~mm}$ in the relative longitudinal positions of $P 1$ and $P 2$, and of $P 3$ relative to $D 1$ and $D 2$, correspond to errors of \pm 0.0002 in $\left\langle\cos \theta_{\mu}\right\rangle$ and $\left\langle\cos \theta_{e}\right\rangle$.

Monte Carlo studies show that the first-order optics formal1sm reconstructs the $\mathrm{e}^{+}$tracks, in the absence of scattering and chamber resolution effects, with an accuracy much better than \pm 0.0001 in. $\left\langle\cos \theta_{e}\right\rangle$. A 108 change in the assumed field strength was shown to cause a change in the reconstructed $\left\langle\cos \theta_{e}\right\rangle=\operatorname{sall}$ compared to 0.0001 . In practice alninizing the wire-chanber rus residuals allowed the field scallng factor [95\$ of the Table (5.1) values] to be duterained to $\pm 5 \$$. A wore conservative estinate of $\$ 0.0002$ for the possible error associated with the firat-ordar optic formalis is adopted hre.

The $\mu^{*}$ and $e^{*}$ have radil of curvature of $-10 \mathrm{a}$ and $-15 \mathrm{a}$ in the

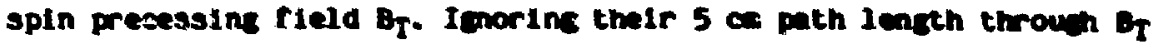
cuses a neglidble error in the reconotructed $\langle 000\rangle^{\prime}$ and $\left\langle 000 e^{2}\right.$. 
The possible reconstruction errors are therefore estimated to be \pm 0.0002 in $\left\langle\cos \theta_{\mu}\right\rangle$ and \pm 0.0003 in $\left\langle\cos \theta_{e}\right\rangle$.

\subsubsection{Moment um Calibration}

The possible errors in the momentum calibration for the various $x$ bins are shown in Table (6.1). Near the $(V-A)$ limit an error $\Delta x$ in momentum yields

$$
\Delta\left[P_{\mu} A(\bar{x})\right] / P_{\mu} A(\tilde{x})=-4 \Delta x /\left(1-4 \tilde{x}^{2}\right)
$$

The mementum calibration contributes a possible error of \pm 0.0010 to the determination of the endpoint asymmetry $P_{\mu} A(0)=\xi P_{\mu} \delta / p[\operatorname{section}(9.4)]$.

\subsubsection{Definition of $x=1$}

In order to fit the data to the theoretical monemtum spectra it is necessary for their endpoints to coincide. This was achieved by fitting the endpolnt positions of both the data and 'events' Benerated from the theoretical spectra, and adjusting the data $x$ to obtain agreement as discussed in section (6.5). Assi gning a possible error of \pm 0.0001 to the endpoint agreement yields an error of $\pm 0.04 \%$ in the fitted asymatries. 1.e. \pm 0.0004 for $P_{p} A(\bar{x})=1$.

\subsubsection{Enercy-Loss Strageling}

An errar of not in the mount of downstrem aterial traversed by

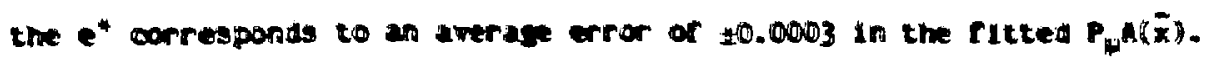




\subsubsection{Muon Mean-Life}

The fits described in section $(7.6)$ were performed with the $u^{+}$ mean-life fixed to the mean value obtained for the corresponding run period: The combined mean-life from the three run periods, which used different clocks, is $\tau_{\mu}=2.209_{ \pm 0.003} \mu \mathrm{s}$ assuming zero background. The: statistical error is $\pm 0.006 \mu$ for free background. A more conservative estimate of \pm 0.008 Hs is adopted here for the possible error in $\tau_{\mu}$. This corresponds to an error of \pm 0.0003 in the fitted $P_{\mu} A(\tilde{x})$.

\subsubsection{Summary}

- The possible systematic errors discussed in sections (8.1) and (8.2) are summarized in Table (8.2). The combined possible systematic error is \pm 0.0013 when averaged over $x$ bins. Table (9.1) shows the possible systematic errors for the individual $x$ bins, which differ due to the monentum calibration contribution. 


\begin{tabular}{|c|c|}
\hline Source of Possible Error & Error \\
\hline Muon depolarization in scattering with $e^{-}$ & \pm 0.0002 \\
\hline Coulomb scattering of muons & \pm 0.0002 \\
\hline Coulomb scattering of positrons & \pm 0.0001 \\
\hline Extra-before muons & \pm 0.0001 \\
\hline Extra-after muons & \pm 0.0003 \\
\hline Cloud muons & \pm 0.0002 \\
\hline Longltudinal field component & \pm 0.0001 \\
\hline Timing errors & \pm 0.0001 \\
\hline Reconstruction of $\theta_{\mu}$ & \pm 0.0002 \\
\hline Reconstruction of $\theta_{e}$ & \pm 0.0003 \\
\hline Momentum calibration & \pm 0.0010 \\
\hline Definition of $x-1$ & \pm 0.0004 \\
\hline Positron energy-loss straggling & \pm 0.0003 \\
\hline Muon mean-iffe $\dot{\tau}_{\mu}$ & \pm 0.0003 \\
\hline Total 10 possible error & \pm 0.0013 \\
\hline
\end{tabular}

Table (B.2) 
Chapter 9

Results and Conclusions

9.1 The Normalized Asymmetries

The weighted mean normalized asymmetries $P_{\mu} A(\tilde{x})$ of the data sets contributing to the final result are shown in Table (9.1). The corrections discussed in section (8.1) are included and the estimated possible systematic errors discussed in section (8.2) are also shown.

\begin{tabular}{ccc}
\hline$x$ Range & $P_{\mu} A(\tilde{x})$ & Systemat 1c Error \\
\hline $0.88-0.90$ & $0.9964 \pm 0.0074$ & \pm 0.0029 \\
$0.90-0.92$ & $1.0109 \pm 0.0062$ & \pm 0.0024 \\
$0.92-0.94$ & $0.9948 \pm 0.0047$ & \pm 0.0018 \\
$0.94-0.96$ & $1.0019 \pm 0.0040$ & \pm 0.0015 \\
$0.96-0.98$ & $0.9939 \pm 0.0034$ & \pm 0.0011 \\
$0.98-1.00$ & $1.0002 \pm 0.0028$ & \pm 0.0009 \\
\hline
\end{tabular}

Table (9.1)

The systemat1c errors Ilsted in Table (9.1) should be regarded as belng completely correlated between the $x$ bins. Thus if the reaults for $N$ of the $x$ bins are conbined the chi-square is given by

$$
x^{2}=\sum_{1 j}\left[\left(p_{1}-d_{1}\right)\left[y^{-1}\right]_{1 j}\left(p_{j}-d_{j}\right)\right.
$$

where

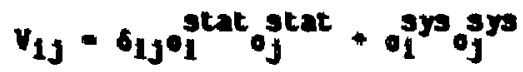




\subsection{Right-Handed Current Limits With Massless Neutrinos}

In left-right symmetric models with massless neutrinos the mass-squared ratio $\varepsilon=M^{2}\left(W_{1}\right) / M^{2}\left(W_{2}\right)$ and mlxing angle $\zeta$ are related to the normalized asymmetries by equation (3.5):

$$
P_{\mu} A(\tilde{x})=1-2\left\{2 \varepsilon^{2}+2 \varepsilon \zeta+\zeta^{2}[1+6 \tilde{x} /(1+\tilde{z} \tilde{x})]\right\}
$$

The right-hand side is unchanged if the replacements $\varepsilon+-\varepsilon$ and $\zeta-\zeta$ are made. Fitting the as ymmetries in Table (9.1) to equation (3.5) therefore yields two minima of equal chi-scyuare $x_{0}^{2}$ in the real $\varepsilon-\zeta$ $\mathrm{plane}$. The physical minimum, denoted by $\left(\varepsilon_{0}, \zeta_{0}\right)$, has $\varepsilon_{0} \geq 0$ whereas $\varepsilon<0$ Implies imaginary $\mathrm{M}\left(\mathrm{W}_{2}\right)$. The $90 \%$ confidence limits $( \pm 1.6450)$ on $\zeta$ for $\varepsilon=\varepsilon_{0}$ correspond to the $\left(\varepsilon_{0}, \zeta\right)$ for which $x^{2}=x_{0}^{2}+2.706$. The contour in Figure (9.1) is a curve of constant $x^{2}-x_{0}^{2}+2.706$ and thus represents a 908 confidence limit in the above sense.

Limits on $M\left(Z_{2}\right)$ are implied by the relation [section (2.2)] $M\left(Z_{2}\right)=M\left(W_{2}\right) \cos \theta_{W} / / V\left(\cos 2 \theta_{W^{\prime}}\right)$. Assuming $M\left(W_{1}\right)=81 \mathrm{GeV} / \mathrm{c}^{2}$ and $\sin ^{2} \theta_{w} \cdot=0.217$ [section (2.1)] the following special case 90 confidence limits are obtained: $M\left(W_{2}\right)>381 \mathrm{GeV} / \mathrm{c}^{2}$ and $M\left(Z_{2}\right)>448 \mathrm{GeV} / \mathrm{c}^{2}$ for any $\zeta$; $M\left(W_{2}\right)>434 \mathrm{GeV} / \mathrm{c}^{2}$ and $M\left(Z_{2}\right)>510 \mathrm{GeV} / \mathrm{C}^{2}$ for $\zeta=0 ;|\zeta|<0.044$ for $M\left(H_{2}\right)=\infty ;$ and $-0.057<\zeta<0.044$ for any $M\left(W_{2}\right)$.

\subsection{Lialts On $H\left(H_{2}\right)$ With $H\left(v_{\mu R}\right)=0$}

The linits obtalned in the preceding section assued asssiess neutrinos. As discussed in section (2.3) a popular model ${ }^{23}$ ) with Hajorana neutrisos thas very heavy $\left[+H\left(W_{2}\right)\right]$ right-handed neutrinos. In

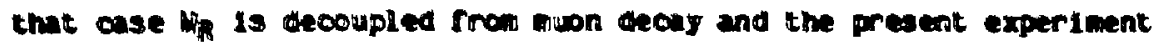




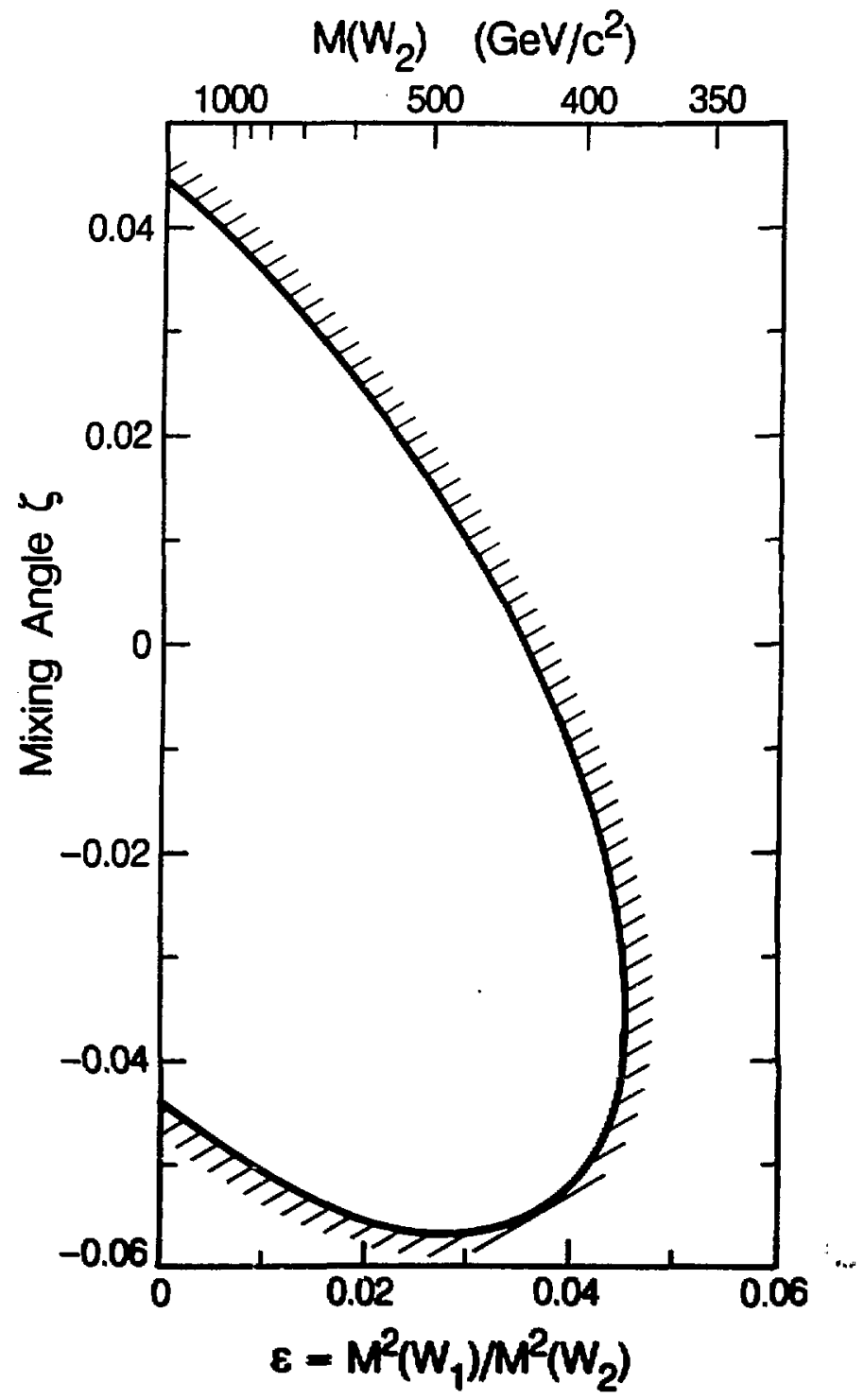

xac tos-11061

FIOURE (9.1). Contour represent 1 ris 903 confidence liaits on the $W_{1,2}$ mas-squared ratio $c$ and the left-right aixins angle 5 . The allowed region contalins $c=t=0$. 
sets no limits on $r i g h t-h a n d e d$ currents. Here limits on $M\left(W_{2}\right)$ are obtained for another possible, if less appealing, scenario: that neutrinos are Majorana particles with $M\left(v_{e R}\right)<<M\left(\nu_{\mu R}\right)<40 \mathrm{MeV} / \mathrm{c}^{2}$. For simplicity it is assumed that the mixing angle $\zeta=0$ so that $W_{2}=W_{R}$.

According to Rekalo5z) the differential decay rate for $\mu^{-}$via $(V-A)$, and hence for $\mu^{+}$via $(V+A)$, including finite $v_{\mu}$ mass, but neglecting $\mathrm{e}^{-}$mass and radiative corrections is

$\frac{d^{2} \Gamma}{d x d(\cos \theta)}-\left(1-v^{2} / k^{2}\right) x^{2}\left\{(3-2 x)+(3-x) \nu^{2} / k^{2}+\cos \theta\left[1-2 x-(1+x) v^{2} / k^{2}\right]\right\}$

where $\nu=M\left(\nu_{\mu}\right), k^{2}=m_{\mu}^{2}-2 m_{\mu} E_{e}$, and $x=E_{e} / E_{e}(\max )$ w1 th $E_{e}(\max )=\left(m_{\mu}^{2}-\nu^{2}\right) / 2 m_{\mu}$. Limits on $M\left(W_{2}\right)$ as a function of $M\left(\nu_{\mu R}\right)$ were determined from the normal1zed asymmetries in Table $(9.1) . M\left(v_{\mu R}\right)=0,14.9,21.1,25.9,29.9$, 33.4 and $36.6 \mathrm{MeV} / \mathrm{c}^{2}$ yleld $W_{R}$-mediated $E_{e}(\max )$ at the $W_{L}$-mediated $x=1,0.98,0.96,0.94,0.92,0.90$, and 0.88 bin boundaries respectively. Considering only the Table :9.1) asymmetries lying below the $W_{R}$-mediated $E_{e}(\max )$ the best fit $(V+A)$ admixture to the $(V-A)$ decay rate was determined for each of the above $M\left(v_{\mu R}\right)$. The $\mu^{+}$from $W_{R}$-mediated $\pi^{+}$decay have momenta too low to be accepted by the beamline for all the above $M\left(\nu_{\mu R}\right)=0$. Since it is assumed here that $\xi=0$ 1t follows that the fitted $(V+\Lambda)$ adnixture is $\varepsilon^{2}$ for the above $M\left(v_{\mu R}\right)=0$, and $2 \varepsilon^{2}$ for $M\left(v_{\mu R}\right)=0$. The unphysical $\varepsilon^{2}<0$ region was excluded and 90\% confidence lower linits on $H_{2}\left(H_{2}\right)$ were deterained In the reaining thysical region.

The result $M\left(H_{2}\right) 2444 \mathrm{GeV} / \mathrm{C}^{2}$ for $I\left(v_{\mu R}\right)=0$ is in close but not perfect agreement with the 1 init $M\left(W_{2}\right)>434$ Gel/ $/ c^{2}$ for 500 and $n(v)=0$ obtalned from the sol confydence c-c contour in section (9.2). 
Accordingly the mass limits found here were reduced by $2 \%$ to establish agreement at $m(v)=0$. The resulting limits on $M\left(W_{2}\right)$ as a function of $M\left(v_{\mu R}\right)$ are shown in Figure (9.2). The kink near $M\left(v_{\mu R}\right)=5 \mathrm{MeV} / \mathrm{c}^{2}$ corresponds to the $W_{R}$-mediated $\pi^{+}$decay $\mu^{+}$momentum decreasing below the beam-line setting as $M\left(\nu_{\mu R}\right)$ increases.

The absence of radiative corrections in quation (9.1) introduces an error into the $M\left(W_{2}\right)$ limits when the $(V-A)$ and $(V+A)$ momenitum spectra have different endpoints, $1 . e=$ when $M\left(v_{\mu R}\right) * 0$. The radiative corrections in section (3.3) reduce the $(V-A)$ decay rate for unpolarized muons by $8.2 \%$ at $x=0.99$ and by $3.5 \%$ at $x=0.89$. Conseçuently in the 'worst case' fit, where the $x=0.978-1.00 W_{R}$-mediated $x$ bin colncldes with the $x=0.88-0.90 \mathrm{~W}_{\mathrm{L}}$-medlated $\mathrm{x}$ bin, the fitted $\varepsilon^{2}$ should be $=5 \%$ too small. Increasing the central value of $\varepsilon^{2}$ by $5 \%$ for the $M\left(\nu_{\mu R}\right)=33.4 \mathrm{MeV} / \mathrm{c}^{2}$ point reduces the corresponding $90 \%$ confidence 1 imit on $M\left(H_{2}\right)$ by only $0.2 \%$. Thus the error introduced by the sosence of radiative corrections in equation (9.1) is negligible.

\subsection{Limits on $E P_{\mu} \delta / \rho$}

The normalized asymetries $P_{\mu} A(\bar{x})$ ar e related to the muon decay paraneters $\varepsilon, \delta$, and $p$ by equation (3.3):

$$
P_{\mu} A(\bar{x})-\left(E P_{\mu} \delta / p\right)(1+2 \bar{x}[\bar{\delta} /(1-\overline{2} \bar{x})-3 \bar{p} /(1+2 \bar{x})]\}
$$

The endpoint asymetry $P_{\mu} \Lambda(0)=E P_{\mu} \delta / p$ was obtalned by fitting the asjmetries in Table (9.1) by equation (3.3) using the world averace

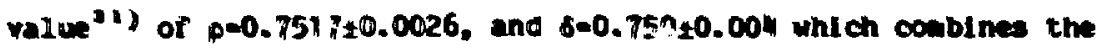

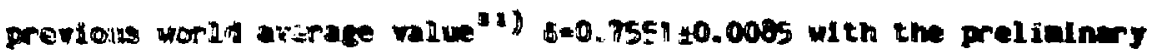




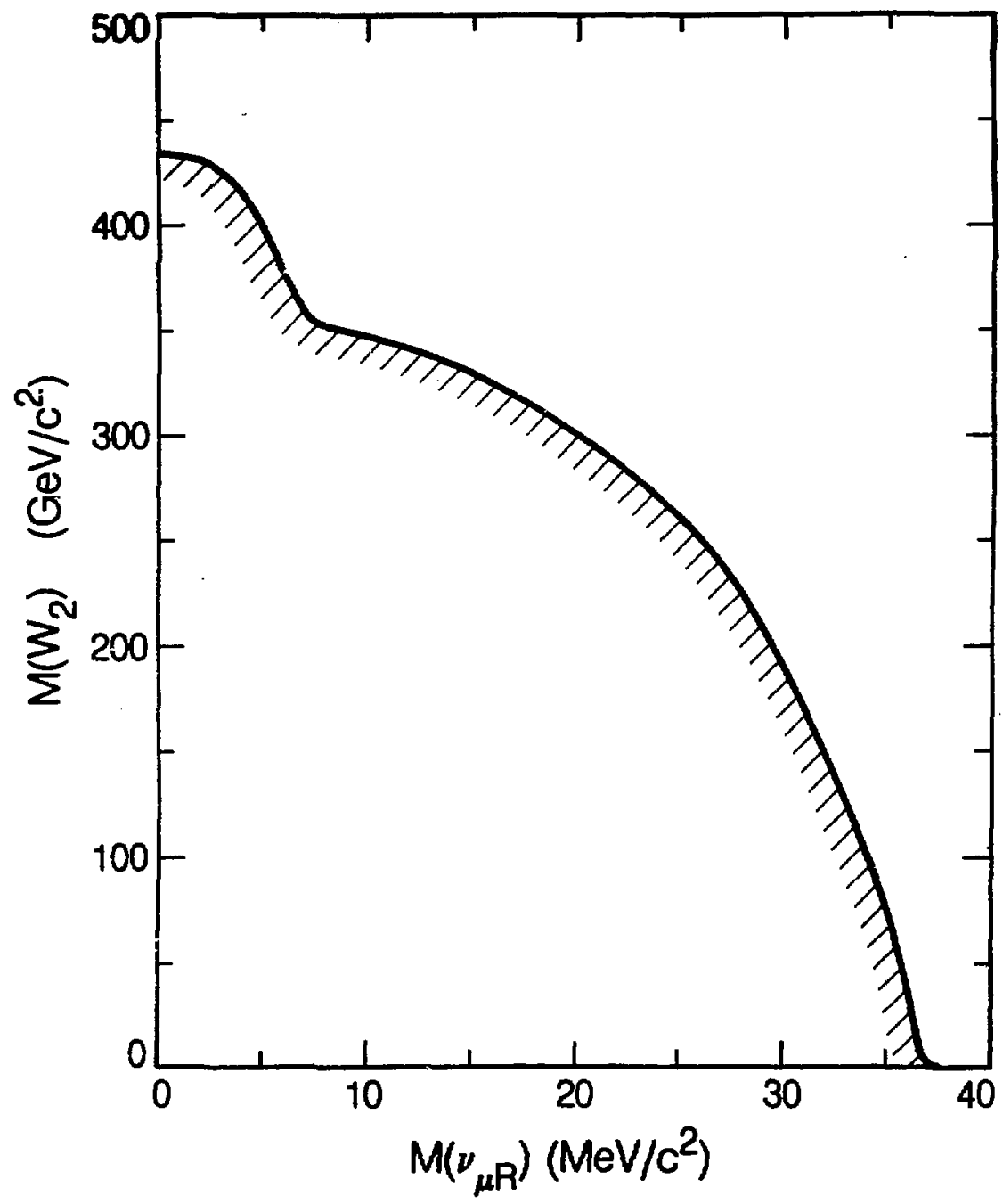

XBL 858-11660

FICURE (9.2). Contour representing $90 \%$ confidence 11 mit on $M\left(H_{2}\right)$ versus the mass of any right-handed $v_{p}$ assuming $M\left(v_{e R}\right)<<M\left(v_{\mu R}\right)$. For $H\left(v_{\mu R}\right)>5 \mathrm{MeV} / \mathrm{C}^{2}$ surface muors from $\mathrm{H}_{R^{-m e d}}$ atated ${ }^{+}$desay have momenta below the beamine momentu acceptance. The allowed region iles above the contour. 
resultss) $\delta=0.748 \pm 0.005$ from Rum 3 of the present experiment. The fit to the asymetries before making the correction of +0.0016 discussed in section (8.1) was shown in Figure (7.4). The uncertainties in $\delta$ and $p$ introduce a possible systematic error of \pm 0.0009 into the determination of $\xi P_{\mu} \delta / \rho$. The fitted value is $E P_{\mu} \delta / \rho=0.9984 \pm 0.0016 \pm 0.0016$. Since any unknown sources of $\mu^{+}$depolarization or any neglected background can only decrease the apparent result, a lower limit for $\xi P_{\mu} \delta / \rho$ should be quoted. Excluding the unphysical $\left(\xi \mathrm{P}_{\mu} \delta / \rho>1\right)$ region the $90 \%$ confidence limit is $\xi P_{\mu} \delta / \rho>0.9951$.

9.5 Limits on $M\left(v_{\mu L}\right)$ and $v_{\mu L}$ Helicity in $\pi^{+}$Decay

Limits on the mass of the left-handed muon neutrino and its helicity in pion decay can be deduced from the $90 \%$ confidence limit $\xi P_{\mu} \delta / \rho>0.9951$. The weakest 1 imits are obtained if it is assumed that right-handed currents are absent. In that case $\xi \delta / \rho=1$ and hence $P_{\mu}>0.9951$. The $90 \%$ confidence limit on the $v_{\mu L}$ helicity in $\pi^{+}$decay is then $\left|h\left(v_{\mu L}\right)\right|>0.9951$. The corresponding limit on the $v_{\mu L}$ velocity $B=v / c>0.9951$ in $\pi^{+}$decay yields the $90 \%$ confidence limit $M\left(v_{\mu L}\right)<3.0 \mathrm{MeV} / \mathrm{c}^{2}$. For comparison the world average value $\left.{ }^{21}\right)$ $M\left(v_{\mu L}\right)<0.5 \mathrm{MeV} / \mathrm{c}^{2}$ implies $P_{\mu}>0.99986$ in the absence of $r i g h t-h a n d e d$ currents. 


\subsection{Lorentz Structure Restrictions}

The couplings in the helicity projection form of the flavor retention interaction Hamiltonian due to Mursula and Scheck ${ }^{3}$ ) are related to $\xi \delta / \rho$ by equation $(3.12)$. If only one coupling other than the $(V-A)$ coupling $B_{22}=1$ is non-zero the $90 \%$ confidence 1 imit $\xi P_{\mu} \delta / p>0.9951$ restricts $\left|g_{11}\right|,\left|f_{11}\right|<0.050$ and $\left|h_{11}\right|,\left|h_{21}\right|<0.10$. The relations amons the couplings under the assumption of $e-p$ uni versality were discussed in section (3.5).

In the special case that the charged current weak interactions are mediated by one heavy spin 1 boson the $\mu^{+}$polarization in $\pi^{+}$decay is given by $P_{\mu}=\left(g_{22}-g_{12}\right) /\left(g_{22}+g_{11}\right)$ and hence $g_{12}<0.0025$ with $90 \%$ confidence.

Mursula and Scheck also considered the case of neutral $Q^{\circ}$ exchange in addition to $W_{L} \pm$ exchange. The $Q^{0}$ would have total lepton number $L=0$ but $L_{e}= \pm 1$ and $L_{\mu}=\mp 1$. With the new scalar, vector, and tensor couplings denoted by $n, \gamma$, and $\phi$ instead of $n, 8$, and $f$ respect 1 vely they find:

$$
\xi \delta / \rho=1-2\left(\left|\gamma_{11}\right|^{2}+\left|\gamma_{12}\right|^{2}+4\left|\phi_{11}\right|^{2}\right)
$$

If only one coupling is non-zero the $90 \%$ confidence limits are $\left|\gamma_{12}\right| .\left|\gamma_{12}\right|<0.050$ and $\left|\phi_{21}\right|<0.025$

\subsection{Limits On Composite Leptons}

The possibility that leptons and quarks are composite at scme mass scale $\mathbf{A}$ has recel ved considerable attention in recent years. Among the strongest experimental limits on $A$ currently quoted 34,35$)$ are those from Bhabba soattering ( $7550 \mathrm{GeV})$, mon ( $\left.\mathrm{B}^{-2}\right)(2860 \mathrm{GeV})$, and a more 
oode1-dependent estimate from v-hadron scattering (>2.5 TeV).

The effects of compositeness may be analyzed in terms of new effective contact interactions. Following the analyses of Peskinss), and Lane and Barany57) the most general $S U(2) \times U(1)$ invariant contact interaction contributing to $\mu+$ ev $\vec{v}$ is

$$
\begin{aligned}
& L_{\text {cont }}=\left(g^{2} / \Lambda^{2}\right)\left[n_{1}\left(\bar{v}_{\mu L} \gamma^{\kappa_{\mu L}}\right)\left(\bar{e}_{L} \gamma_{K} v_{e L}\right)+n_{2}\left(\bar{v}_{\mu R} \gamma \kappa_{\mu R}\right)\left(\bar{e}_{R} \gamma_{K} v_{e R}\right)\right. \\
& +n_{3}\left(\bar{v}_{\mu L} \gamma^{K} v_{e L}\right)\left(\bar{e}_{R} \gamma_{K} \mu_{R}\right)+n_{L}\left(\bar{e}_{L} \gamma^{K} \mu_{L}\right)\left(\bar{v}_{\mu R} \gamma_{K} v_{e R}\right) \\
& +n_{s}\left(\bar{v}_{\mu L \mu_{R}}\right)\left(\bar{e}_{L} \nu_{e R}\right)+n_{6}\left(\bar{v}_{\mu L} \nu_{e R}\right)\left(\bar{e}_{L} \mu_{R}\right) \\
& +n_{7}\left(\bar{v}_{\mu R \mu_{L}}\right)\left(\bar{e}_{R} v_{e L}\right)+n_{0}\left(\bar{v}_{\mu R} v_{e L}\right)\left(\bar{e}_{R} \mu_{L}\right)
\end{aligned}
$$

where $g$ is a coupling of hadronic strength; the $n_{i}$ are of order unity and are normalized so that $\left|n_{L}\right|=1$ in the diagonal couplinis

$$
\left(g^{2} / 2 \Lambda^{2}\right)\left[n_{L}\left(\bar{e}_{L} \gamma^{k} e_{L}\right)\left(\bar{e}_{L} \gamma_{K} e_{L}\right)+\ldots\right]
$$

The first and second terms in equation (9.3) are purely left-nanded and $r i g h t$-handed respect 1 vely, and hence are indistinguishable from the usual. $(V-A)$ and $(V+A)$ interactions.

There are three special cases of interest:

1. If only left-handed ( $r$ ight-handed) leptons are composite then only the purely left-handed (right-handed) term survives, 1.e. only $n_{1}\left(n_{2}\right) * 0$

2. If beth left-handed and right-handed leptons are composite but contaln quite different sets of constituents then the purely Left-handed and right-handed terns dominate, 1 ,e. $n_{1}, n_{2}>$ sother $n_{1}$. 3. If there is $n_{0} v_{R}$, or $M\left(v_{R}\right)$ is large, only $n_{1}, n_{3}=0$. Assuling an effective Interaction Lagrandian Leff - Ly-A + Lcont yields the endpoint decay rate: 


$$
1-P_{\mu} A(0)=2(620 G e V / A)^{4}\left(g^{2} / 4 \pi\right)^{2}\left(n_{2}^{2}+n_{3}^{2}+n_{s}^{2} / 4\right)
$$

The Iimit $P_{\mu} A(0)=\xi P_{\mu} \delta / p>0.9951$ then Implies

$$
\Lambda^{2}>(2780 \mathrm{GeV})^{2}\left(\mathrm{~g}^{2} / 4 \pi\right) V\left(n_{2}^{2}+n_{3}^{2}+n_{5}^{2} / 4\right)
$$

with $90 \%$ confidence. (If the not unreasonable assumptions $g^{2} / 4 \pi=2.1$ and $\Pi_{1}>0.2$ are made, the 1 imit $\Lambda>2200 \mathrm{GeV}$ would be obtained.)

For the special cases discussed earlier the limit becomes

1. Only left-handed leptons composite: no limit.

only right-handed leptons composite: $\quad \Lambda^{2}>(2780 \mathrm{GeV})^{2}\left(\mathrm{~g}^{2} / 4 \pi\right) n_{2}$

2. Left- and right-handed leptons have

different sets of constituents: $\left.\quad \Lambda^{2}>(2780 \mathrm{GeV})^{2} i^{2} / 4 \pi\right) n_{2}$

3. No $v_{R}$, or $M\left(v_{R}\right)$ large:

$\Lambda^{2}>(2780 G e V)^{2}\left(g^{2} / 4 \pi\right) \eta_{3}$ 


\section{Ippendix A}

\section{First-Order Opties of Solenoldal Fields}

This Appendix follows closely a set of notes by $K$. Halbach $\left.{ }^{4}\right)$. The equation of motion for a particle of momentum $\mathrm{p}$ and charge $\mathrm{e}$ in an external magnetic field $\mathrm{B}$ is

$$
\dot{p}=e(\underset{\sim}{\dot{x}} \times \underset{\sim}{B})
$$

Evaluation of $\underset{\nabla}{B}=0$ on the solenold axis (z-axis) gives the first order off-axis field components

$$
B_{x}=-x B_{z} 1 / 2 \text { and } B_{y}=-y B_{z} / / 2
$$

where $d / d z$ is denoted by '.

Then from (A.1)

$$
\begin{aligned}
& \dot{p}_{x}=e\left(\dot{y}_{z}+\dot{z} y B_{z}{ }^{\prime / 2}\right) \\
& \dot{p}_{y}=-e\left(\dot{z} x B_{z}{ }^{\prime / 2}+\dot{x} B_{z}\right) \\
& \dot{p}_{z}=e(\dot{y} x-\dot{x} y) B_{z}{ }^{\prime / 2}
\end{aligned}
$$

With $\dot{z}-v_{0}$ and $e B_{z} / m y_{0}=B_{z} / B p=k$, where $B p$ is the magnet ic rigidity of the particle, (A.2) and (A.3) become

$$
\begin{aligned}
& x^{\prime \prime}=y^{\prime} k+y k^{\prime} / 2 \\
& y^{\prime \prime}=-\left(x^{\prime} k+x k^{\prime} / 2\right)
\end{aligned}
$$

which with the notation $u=x+i y$ may be written as

$$
w^{m}=-1\left(k w^{\prime}+k^{\prime} w / 2\right)
$$


Introducing a new coordinate system $\zeta=\xi+1 n$ in the w plane, but rotated by $-\alpha$ with respect to $w=x+1 y$ gives

$$
\begin{aligned}
& w=\zeta e^{1 \alpha} \\
& w^{\prime}=\left(\zeta^{\prime}+1 \alpha^{\prime} \zeta\right) e^{1 \alpha} \\
& w^{\prime \prime}=\left(\zeta^{\prime \prime}+21 \alpha^{\prime} \zeta^{\prime}+1 \alpha^{n} \zeta-\alpha^{\prime 2} \zeta\right) e^{1 \alpha}
\end{aligned}
$$

and from (A.5)

$$
\zeta^{\prime \prime}+1\left(2 \alpha^{\prime}+k\right) \zeta^{\prime}+\left(1 \alpha^{\prime \prime}-\alpha^{\prime 2}-\alpha^{\prime} k^{\prime}+1 k^{\prime} / 2\right) \zeta=0
$$

Now setting $\alpha^{\prime}=-k / 2, \quad \alpha=-(1 / 2 B \rho) \int_{0}^{z} B_{Z}(z) d z$ yields

$$
\zeta^{\prime \prime}+(k / 2)^{2} \zeta=0
$$

The particle motions in the $\xi$ and $\eta$ directions of the rotating $\zeta$ coordinate systen are now decoupled:

$$
\xi^{\prime \prime}+(k / 2)^{2} \xi=0 \text { and } \eta^{\prime \prime}+(k / 2)^{2} \eta=0
$$

Equation (A.8) has solution

$$
\zeta(z)=c_{1} \cos (k z / 2)+c_{2} \sin (k z / 2)
$$

and hence

$$
\zeta^{\prime}(z)=(k / 2)\left[-c_{2} \sin (k z / 2)+c_{2} \cos (k z / 2)\right]
$$

Choosing the initial conditions $\zeta(0)=\zeta_{0}$ and $\zeta^{\prime}(0)=\zeta_{0} 0^{\prime}$ implies $c_{2}=\zeta_{0}$ and $c_{2}=2 \zeta_{0} \cdot k$. Thus $\left(\zeta, \zeta^{\prime}\right)$ at $z+L$ are related to $\left(\zeta_{0}, \zeta_{0}+\right)$ at 2 by

$$
\left[\begin{array}{l}
5 \\
5^{\prime}
\end{array}\right]=\left[\begin{array}{lr}
\cos (k L / 2) & (2 / k) \sin (k L / 2) \\
-(k / 2) \sin (k L / 2) & \cos (k L / 2)
\end{array}\right]\left[\begin{array}{l}
\zeta_{0} \\
5_{0}
\end{array}\right]
$$


where $k=\left\langle B_{2}\right\rangle / B p$.

The track vector in the laboratory ( $(H)$ coordinate systed is giren

by $(\Lambda .6)$ and $(A .7)$ :

$$
\begin{aligned}
x+1 y & =(\xi+1 \eta)(\cos \alpha+i \sin \alpha) \\
x^{\prime}+i y^{\prime} & =\left[\xi^{\prime}+i \eta^{\prime}+(\eta-i \xi) k / 2\right](\cos \alpha+i \sin \alpha)
\end{aligned}
$$

Transport matrices between the stopping target and the wire planes of P3-D2 were formed by multiplying together the transport matrices of (A.9) corresponding to successive short steps along the solenold axis using the fleld values in Table (5.1). The inftial $e^{+}$track vector at the stopping target may then be determined from a least squares $f 1 t$ to the wire chamber space points. 


\section{Appendix $B$}

\section{Positron Energy-Loss Straggling}

The $\mathrm{e}^{+}$lose energy by ionization (Including Bhabba scattering) and bremsstrahlung. The lonization energy-loss $\Delta E$ has a mach shorter tail than the bremsstrahlung, faling as $1 /(\Delta E)^{2}$ versus $1 / \Delta E$ for the bremsstrahlung. Comparison of the formulae given by Tsai"s) shows that the Ionization (bremsstrahlung) process dominates for $\Delta E$ less (greater) than about $20-\mathrm{MeV} /(Z+2.5)$ where $\mathrm{Z}$ is the atomic number of the material. Since the $\mu S R$ data $x$ range of $0.88-1.00$ corresponds to an energy range of $6.3 \mathrm{MeV}$ both processes must be considered.

According to Tsai ${ }^{49}$ ) the probability that an electron with initial energy $E_{0}$ has energy $E^{\prime}>E_{0}-\Delta_{0}-\Delta E$ after traversing $t$ radiation lengths, where $\Delta_{0}$ is the most probable energy-loss due to lonlzation, is

$$
P\left(E_{0}, E^{\prime}, t\right)=(1+0.5772 b t)\left[\frac{\Delta E}{E_{0}}\right]^{b t}\left[1-\frac{r}{(1-b t) \Delta E}\right]
$$

where $\quad T=0.154 \mathrm{MeV}(Z / A) \mathrm{g}$

with $\quad g=$ number of $g / \mathrm{cm}^{2}$ for $t$ radiation lengths

and $\quad b=(4 / 3)\left[1+(z+1) / 9(z+n) \ln \left(183 z^{-1 / 3}\right)\right]$

with $n=\ln \left(1440 z^{-2 / 3}\right) / \ln \left(183 z^{-1 / 3}\right)$

It follows from equation (B.1) that the probability of the straggled energy lying in the range $E_{0}-\Delta_{0}-\Delta E_{1}<E^{h}<E_{0}-\Delta_{0}-\Delta E_{2}$ is $P\left(E_{1}, E^{n}, t\right)=\frac{1+0.5772 b t}{E_{0} b t}\left[\left[\Delta E_{2}^{b t}-\Delta E_{2}^{b t}\right]-\frac{T}{1-b t}\left[\Delta E_{2}^{b t-1}-\Delta E_{2}^{b t-1}\right]\right\} \quad$ (B.2) 
(3.3)] was evaluated for $\cos \theta=-1,0,1$ at monent un intervals of $\Delta x=0.0004$ in the range $x=0.88-1.00$. These three monentum spectra were straggled according to equation (B.2) 1 gnoring the most probable ionization energy-loss $\Delta_{0}$ which is essentially constant over the $x$ range of interest. Equation (B.2) is valid for $\triangle E Z 10 r$. Consequently the stopping target material and the other material upstream of the spectrometer traversed by the $e^{+}$were each divided into 10 steps and the straggling was performed by successive application of equation (B.2). 


\section{Appendi $x$ C}

Tables of Data Fit Results

$\begin{array}{lll}\text { Run Period } \quad: & 1 \\ \text { Target } \quad: & \mathrm{Ag} \\ \mathrm{B}_{\mathrm{T}} & 70-\mathrm{G} \\ \text { Events Fitted: } & 24457\end{array}$

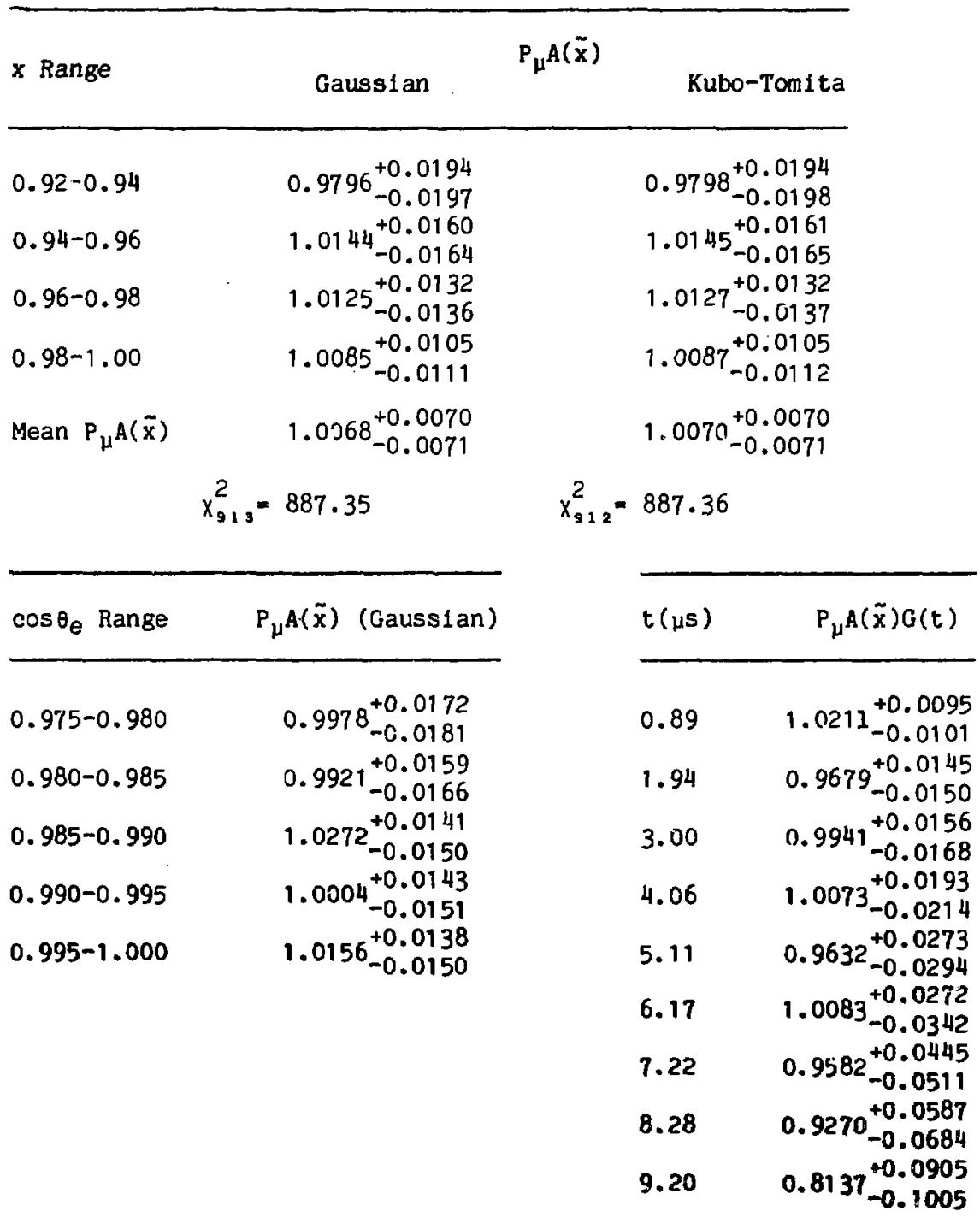




$\begin{array}{lll}\text { Run Period } & : & 1 \\ \text { Target } & : & A 1 \\ \text { BT } & : & 70-G \\ \text { Events Fitced: } & 27410\end{array}$

\begin{tabular}{|c|c|c|}
\hline x Range & Gaussian & Kubo-Tomita \\
\hline $0.92-0.94$ & $0.9928_{-0.0194}^{+0.0191}$ & $0.9980^{+0.0211}$ \\
\hline $0.94-0.96$ & $1.0006_{-0.0160}^{+0.0156}$ & $1.0055_{-0.0172}^{+0.0182}$ \\
\hline $0.96-0.98$ & $0.9842_{-0.0135}^{+0.0131}$ & $0.9896_{-0.0151}^{+0.0173}$ \\
\hline $0.98-1.00$ & $0.9743_{-0.0121}^{+0.0116}$ & $0.9798_{-0.0138}^{+0.0168}$ \\
\hline \multirow[t]{2}{*}{ Mean $P_{\mu} A(\tilde{x})$} & $0.9849^{+0.0071}$ & $0.9927_{-0.0087}^{+0.0089}$ \\
\hline & 916.57 & 915.73 \\
\hline
\end{tabular}

cos $\theta_{e}$ Range $\quad P_{\mu} A(\tilde{x})$ (Gaussian)

$0.975-0.980$

$0.980-0.985$

$0.985-0.990$

0.990-0.995

$0.995-1.000$

$$
\begin{array}{r}
1.0024^{+0.0156}-0.0165 \\
+0.0140 \\
1.0081+0.0148 \\
-0.0143 \\
0.9701^{+0.0143}-0.0149 \\
+0.0150 \\
0.9728+0.0156 \\
0.9699^{+0.0169}-0.0176
\end{array}
$$

$t(\mu s) \quad P_{\mu} A(\tilde{x}) G(t)$

$\begin{array}{lr}0.89 & 0.9867_{-0.0105}^{+0.0101} \\ 1.94 & 0.9817_{-0.0134}^{+0.0128} \\ 3.00 & 0.9585_{-0.0175}^{+0.0167} \\ 4.06 & 0.9792_{-0.0209}^{+0.0194} \\ 5.11 & 0.9184_{-0.0295}^{+0.0276} \\ 6.17 & 0.9012_{-0.048}^{+0.0388} \\ 7.22 & 0.9325_{-0.0533}^{+0.0490} \\ 8.28 & 0.9135_{-0.0624}^{+0.0561} \\ 9.20 & 0.9754_{-0.0605}^{+0.0675}\end{array}$


$\begin{array}{lll}\text { fin Period } & : & 1 \\ \text { Target } & \text { Au } \\ \text { B }_{\text {T }} & : & 70-\mathrm{G}\end{array}$

Events Fitted: 20174

x Range Gaussian $\quad P_{\mathcal{H}} A(\tilde{x}) \quad$ Kubo-Tomita
$0,92-0.94$
$1.0051+0.0209$
$1.0051+0.0209$
$0.94-0.96$
$1.0357_{-0.0179}^{+0.0174}$
$1.0357_{-0.0180}^{+0.0175}$
$0.96-0.98$
$0.9957_{-0.0151}^{+0.0146}$
$0.9957_{-0.0151}^{+0.0146}$
$0.98-1.00$
$0.9951+0.0120$
$0.9951+0.0120$

Mean $P_{\mu} A(\bar{x}) \quad 1.0040_{-0.0077}^{+0.0077}$

$1.0040_{-0.0077}^{+0.0077}$

$x_{913}^{2}=1015.16$

$x_{912}^{2}=1015.18$

$\cos \theta_{e}$ Range $\quad P_{\mu} A(\tilde{x})$ (Gaussian)

$t(\mu s) \quad P_{\mu} A(\tilde{x}) G(t)$

$\begin{array}{lr}0.975-0.980 & 1.0223_{-0.0177}^{+0.0164} \\ 0.980-0.985 & 0.9931^{+0.0165}-0.0174 \\ 0.985-0.990 & 1.0046^{+0.0160}-0.0170 \\ 0.990-0.995 & 1.0179_{-0.0167}^{+0.0167} \\ & 0.0199^{+0.0170} \\ 0.995-1.000 & 0.0184\end{array}$

$0.89 \quad 0.9815_{-0.0124}^{+0.0119}$

$1.94 \quad 1.0205+0.0122$

$3.00 \quad 0.9797_{-0.0189}^{+0.0176}$

$4.06 \quad 1.0216_{-0.0238}^{+0.0218}$

$\begin{array}{lll}5.11 & 1.0357_{-0.0227}^{+0.0150}\end{array}$

$6.17 \quad 0.9078-0.0431$

$7.22 \quad 0.9075+0.0548$

$8.280 .9456=0.0723$

$9.20 \quad 0.6744^{+0.1176}$ 


$\begin{array}{lll}\text { Run Period } & : & 1 \\ \text { Target } & : & \mathrm{C}_{-} \\ \mathrm{B}_{\mathrm{T}} & \mathbf{7 0 - \mathrm { G }} \\ \text { Events Fitted: } & 23734\end{array}$

\begin{tabular}{|c|c|c|c|}
\hline$x$ Range & Geussian & $P_{\mu} A(\tilde{x})$ & Kubo-Tomita \\
\hline $0.92-0.94$ & $0.9930^{+0.0195}$ & $;$ & $0.9930_{-0.0199}^{-0.0195}$ \\
\hline $0 n n-0.96$ & $0.9904_{-0.0171}^{+0.0167}$ & & $0.9905_{-0.0171}^{+0.0167}$ \\
\hline $0.96-0.98$ & $1.0004_{-0.0142}^{+0.0138}$ & & $1.0005_{-0.0143}^{+0.0138}$ \\
\hline $0.98-1.00$ & $1.0145_{-0.0104}^{+0.0097}$ & & $1 .\left(145^{+0.0097}\right.$ \\
\hline Mean $P_{\mu} A(\tilde{x})$ & $1.0040_{-0.0070}^{+0.0069}$ & & $1 . n 041+0.0069$ \\
\hline & 936.60 & $x_{9}^{2}$ & 936.60 \\
\hline
\end{tabular}

\begin{tabular}{|c|c|}
\hline$t(\mu s)$ & $P_{\mu} A(\tilde{x}) G(t)$ \\
\hline 0.89 & $0.9988_{-0.0114}^{+0.01 C}$ \\
\hline$\therefore 94$ & $1.0078_{-0.0135}^{+0.0126}$ \\
\hline 3.00 & $0.9890_{-0.0169}^{+0.0156}$ \\
\hline 4.06 & $0.59 .99+0.0201$ \\
\hline 5.11 & $0.9841_{-0.0287}^{+0.0314}$ \\
\hline 6.17 & $1.0156_{-0.0395}^{+0.0359}$ \\
\hline 7.22 & $\begin{array}{l}.0471 \\
0.0538\end{array}$ \\
\hline 8.28 & $\begin{array}{l}.0644 \\
.0717\end{array}$ \\
\hline 9.20 & $0.8932^{\div 0.0 .0852}$ \\
\hline
\end{tabular}


$\begin{array}{lll}\text { Rur Perlod } & : & 1 \\ \text { Target } & : & \text { He } \\ \text { BT } & 70-G \\ \text { Events Fitted: } & 28547\end{array}$

\begin{tabular}{|c|c|c|}
\hline$x$ Range & Gauss 1an & Kubo-Ton1 ta \\
\hline $0.92-0.94$ & $0.8645_{-0.0212}^{+0.0209}$ & $0.9124_{-0.0247}^{+0.0246}$ \\
\hline $0.94-0.96$ & $0.8835_{-0.0184}^{+0.0183}$ & $0.9321+0.0220$ \\
\hline $0.96-0.98$ & $0.8906_{-0.0162}^{+0.0160}$ & $0.9396_{-0.0199}^{+0.0198}$ \\
\hline $0.98-1.00$ & $0.8653_{-0.0156}^{+0.0153}$ & $0.9147+0.0191$ \\
\hline Mean $P_{\mu} A(\tilde{x})$ & $0.8764^{+0.0087}$ & $0.9252+0.0106$ \\
\hline & 910.98 & 906.92 \\
\hline
\end{tabular}

\section{$\cos \theta_{e}$ Range $\quad P_{1} A(\tilde{x})$ (Gaussian)}

$\begin{array}{lr}0.975-0.980 & 0.8956_{-0.0202}^{+0.0196} \\ 0.980-0.985 & 0.8715_{-0.0194}^{+0.0190} \\ 0.985-0.990 & 0.8511_{-0.0189}^{+0.0186} \\ 0.990-0.995 & 0.8900_{-0.0187}^{+0.0183} \\ 0.995-1.000 & 0.8791_{-0.0206}^{+0.0201}\end{array}$

$t(\mu s) \quad P_{\mu} A(\tilde{x}) G(t)$

$0.89 \quad 0.8912_{-0.0118}^{+0.0115}$

$1.94 \quad 0.8042+0.0161$

$3.00 \quad 0.8322+0.0202$

$4.06 \quad 0.7975_{-0.0267}^{+0.0259}$

$5.110 .7208+0.0359$

$6.17 \quad 0.6660+0.0467$

$7 . c 2 \quad 0.6550+0.0589$

$8.28 \quad 0.4992-0.0827$

$9.20 \quad 0.5976_{-0.1168}^{+0.1107}$ 


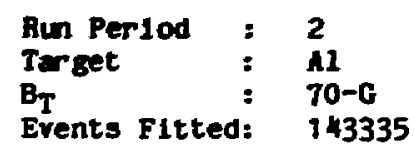

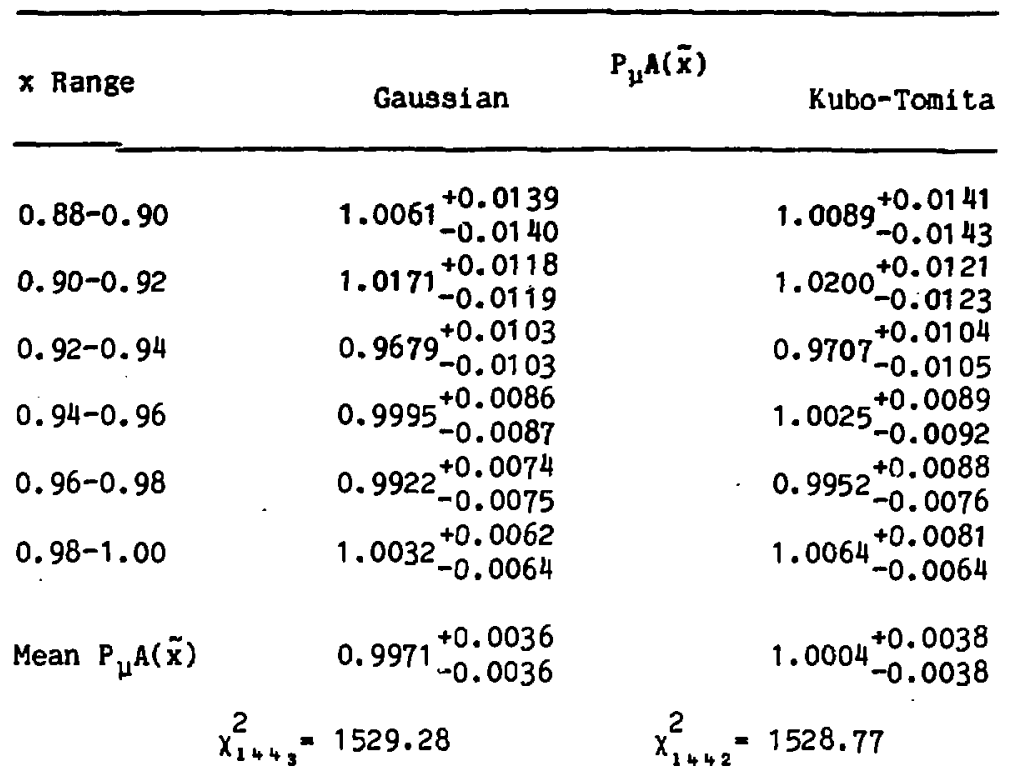

\begin{tabular}{|c|c|c|c|}
\hline $\cos \theta_{e}$ Range & $P_{\mu} A(\tilde{x})$ (Gaussian) & $t(\mu s)$ & $P_{\mu} A(\tilde{x}) G(t)$ \\
\hline $\begin{array}{l}0.975-0.980 \\
0.980-0.985 \\
0.985-0.990 \\
0.990-0.995 \\
0.995-1.000\end{array}$ & $\begin{array}{r}0.9844+0.0109 \\
-0.0112 \\
+0.0082 \\
0.9925_{-0.0083} \\
+0.0074 \\
1.0081+0.0076 \\
-0.0076 \\
+0.0072 \\
0.9992=0.0073 \\
0.9936^{+0.0071}=0.0072\end{array}$ & $\begin{array}{l}0.64 \\
1.70 \\
2.76 \\
3.82 \\
4.87 \\
5.93 \\
6.99 \\
8.05 \\
9.08\end{array}$ & 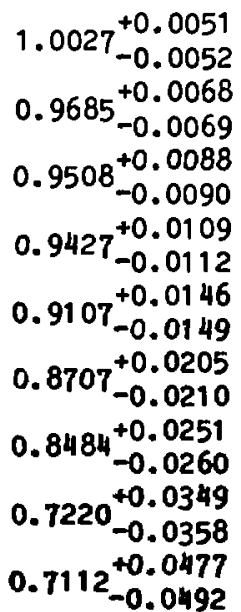 \\
\hline
\end{tabular}

Tatie (C.1) cont. 


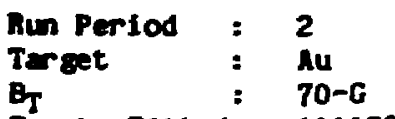

Events Fitted: $1: 1158$

\begin{tabular}{|c|c|c|}
\hline x Range & Gaussian & Kubo-Tomita \\
\hline $0.88-0.90$ & $1.0188_{-0.0152}^{+0.0150}$ & $1.0195_{-0.0155}^{+0.0153}$ \\
\hline $0.90-0.92$ & $1.0250_{-0.0125}^{+0.0124}$ & $1.0257_{-0.0129}^{+0.0129}$ \\
\hline $0.92-0.94$ & $0.9839^{+0.0108}$ & $0.9846_{-0.0113}^{+0.0113}$ \\
\hline $0.94-0.96$ & $0.9976_{-0.0093}^{+0.0092}$ & $0.9983+0.0100$ \\
\hline $0.96-0.98$ & $\begin{array}{l}0.9924^{+0.0078} \\
-0.0080\end{array}$ & $0.9931^{+0.0095}$ \\
\hline $0.98-1.00$ & $0.9949+0.0063$ & $0.9957_{-0.0072}^{+0.0080}$ \\
\hline Mean $P_{\mu} A(\tilde{x})$ & $0.9975_{-0.0037}^{+0.0037}$ & $0.9989_{-0.0042}^{+0.0043}$ \\
\hline & 1510.97 & 1510.99 \\
\hline
\end{tabular}

$\cos \theta_{e}$ Range $\quad P_{\mu} A(\tilde{x})$ (Gaussian)

$t(\mu s) \quad P_{\mu} A(\tilde{x}) G(t)$
$0.975-0.980$
$0.9905_{-0.0117}^{+0.0114}$
0.64
$0.9989+0.0057$
$0.980-0.985$
$1.0129+0.0085$
1.70
$0.9944+0.0072$
$0.985-0.990$
$0.9911_{-0.0082}^{+0.0080}$
2.76
$0.9916_{-0.0092}^{+0.0089}$
$0.990-0.995$
$0.9922+0.0075$
$3.82 \quad 0.9981_{-0.0120}^{+0.0115}$
$3.82 \quad 0.9981_{-0.0120}^{+0.0115}$
$0.995-1.000$
$0.9989+0.0074$
4.87
$0.9978+0.0138$
$5.93 \quad 0.9888_{-0.0194}^{+0.0182}$
6.99
$1.0065+0.0213$
8.05
$0.9231+0.0344$
9.08
$1.0166+0.0343$

Table (C.1) cont. 


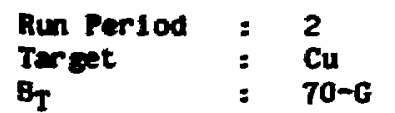

Events Fitted: 129820

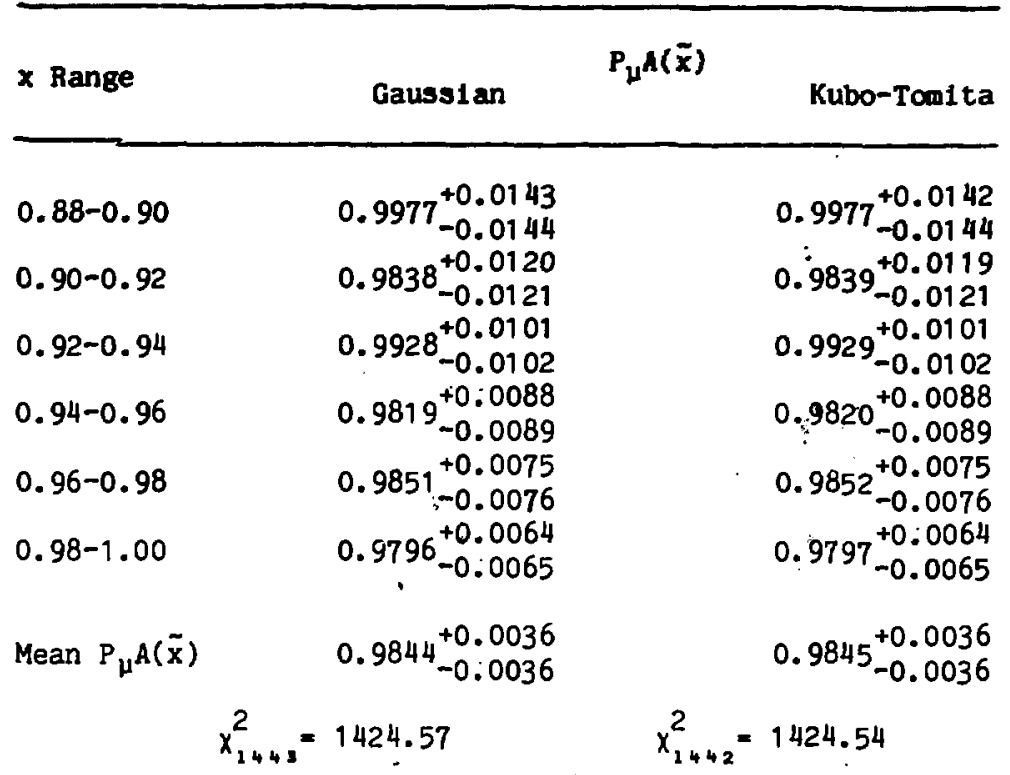

\begin{tabular}{|c|c|c|c|}
\hline $\cos \theta_{e}$ Range & $P_{\mu} A(\tilde{x})$ (Gaussian) & $t(\mu s)$ & $P_{\mu} A(\tilde{x}) G(t)$ \\
\hline $\begin{array}{l}0.975-0.980 \\
0.980-0.985 \\
0.985-0.990 \\
0.990-0.995 \\
0.995-1.000\end{array}$ & $\begin{array}{r}0.9865_{-0.0090}^{+0.0088} \\
+0.0082 \\
0.9823_{-0.0084}^{+0.008} \\
0.9915_{-0.0077}^{+0.0077} \\
0.9806_{-0.0075}^{+0.0075} \\
0.0076 \\
0.9866_{-0.0072}^{+0.0071}\end{array}$ & $\begin{array}{l}0.64 \\
1.70 \\
2.76 \\
3.82 \\
4.87 \\
5.93 \\
6.99 \\
8.05 \\
9.08\end{array}$ & 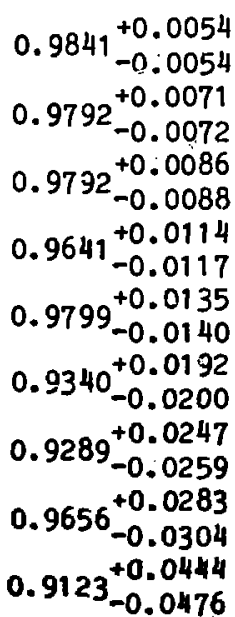 \\
\hline
\end{tabular}

Table (C.1) cont. 
Run Perlod 2; Target $=$ Al; $B_{\mathrm{T}}=110-\mathrm{G}_{;}$Events Fitted $=58529$

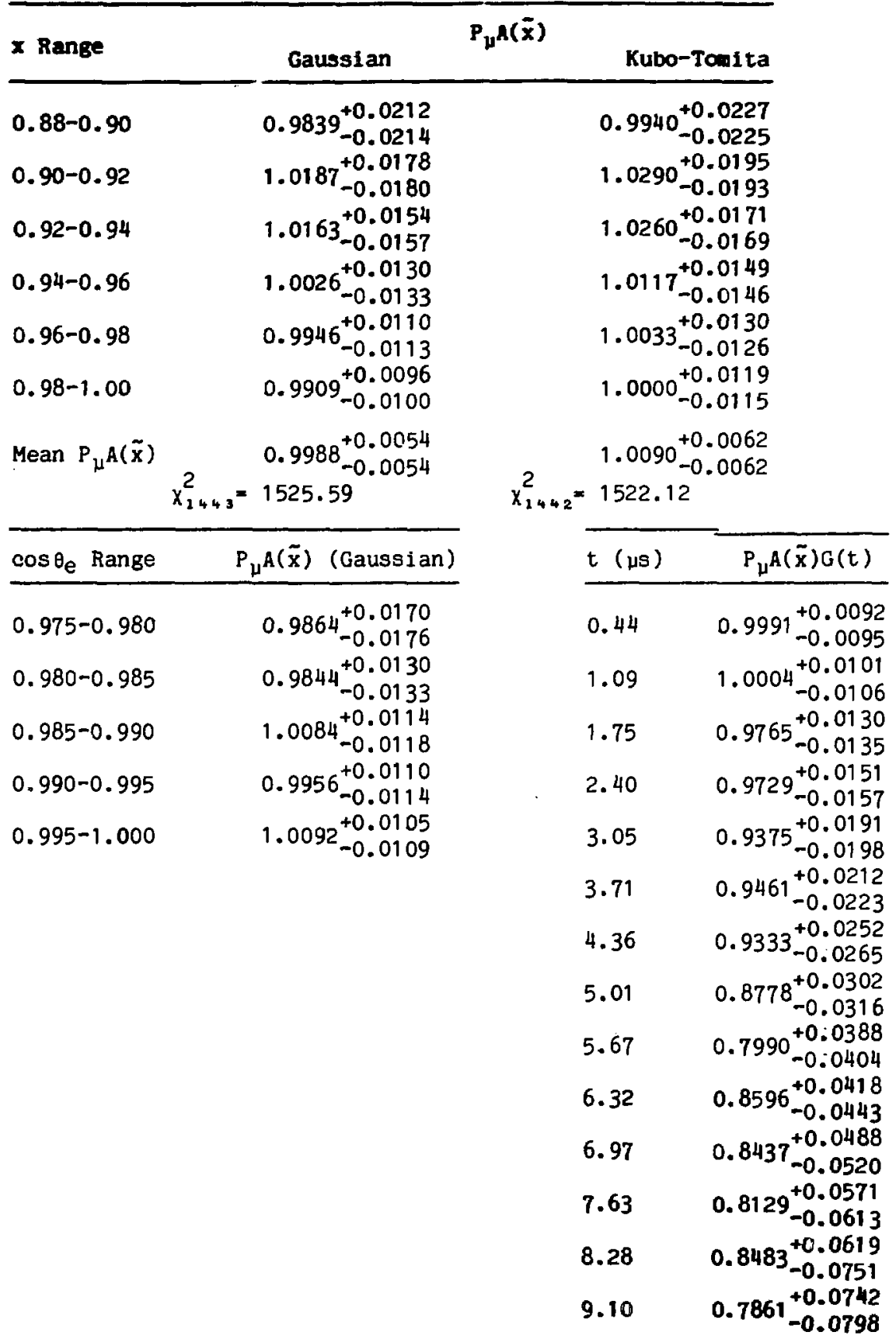

Table (C.1) cont. 
Aun Period 2; Tareet $=\Lambda 1^{*} ; B_{T}=110-G ;$ Events P1tted $=55445$

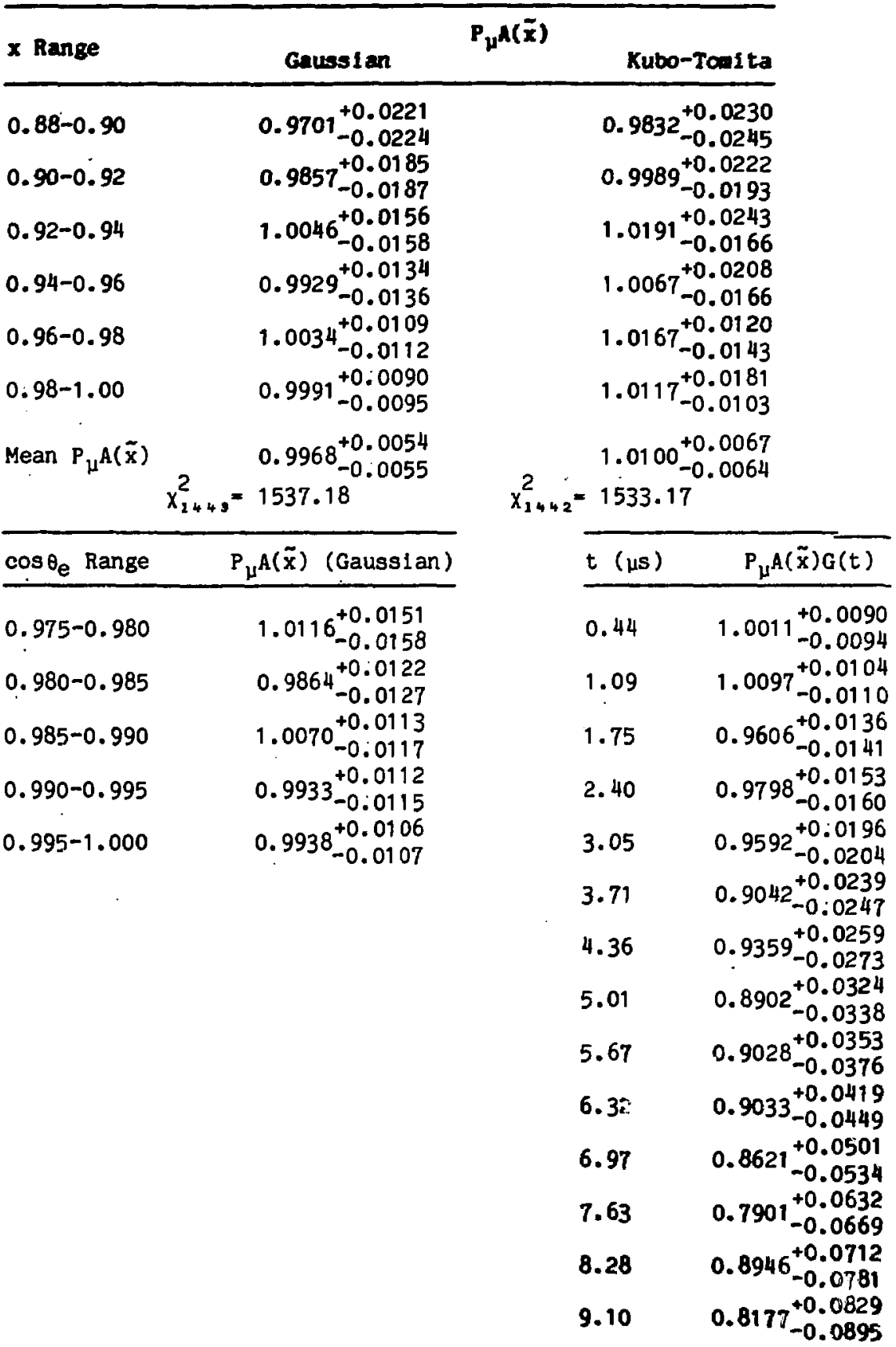

Table (C.I) cont. 
Run Period 2; Target - Au; $B_{T}=110-G ;$ Events Fitted $=28456$

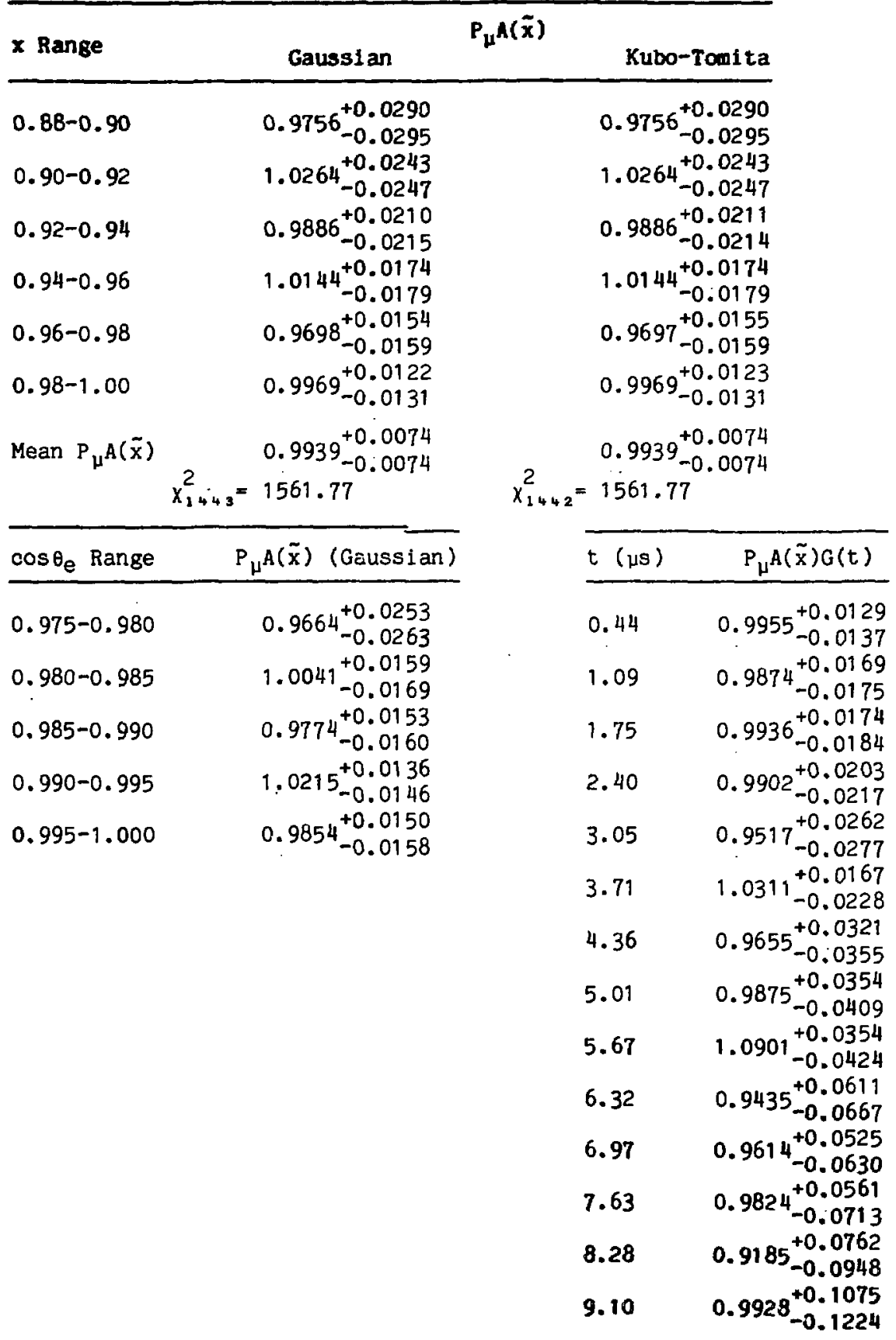


Run Perlod 2; Target $=$ Cu; $B_{T}=110-G ;$ Events Fitted $=41924$

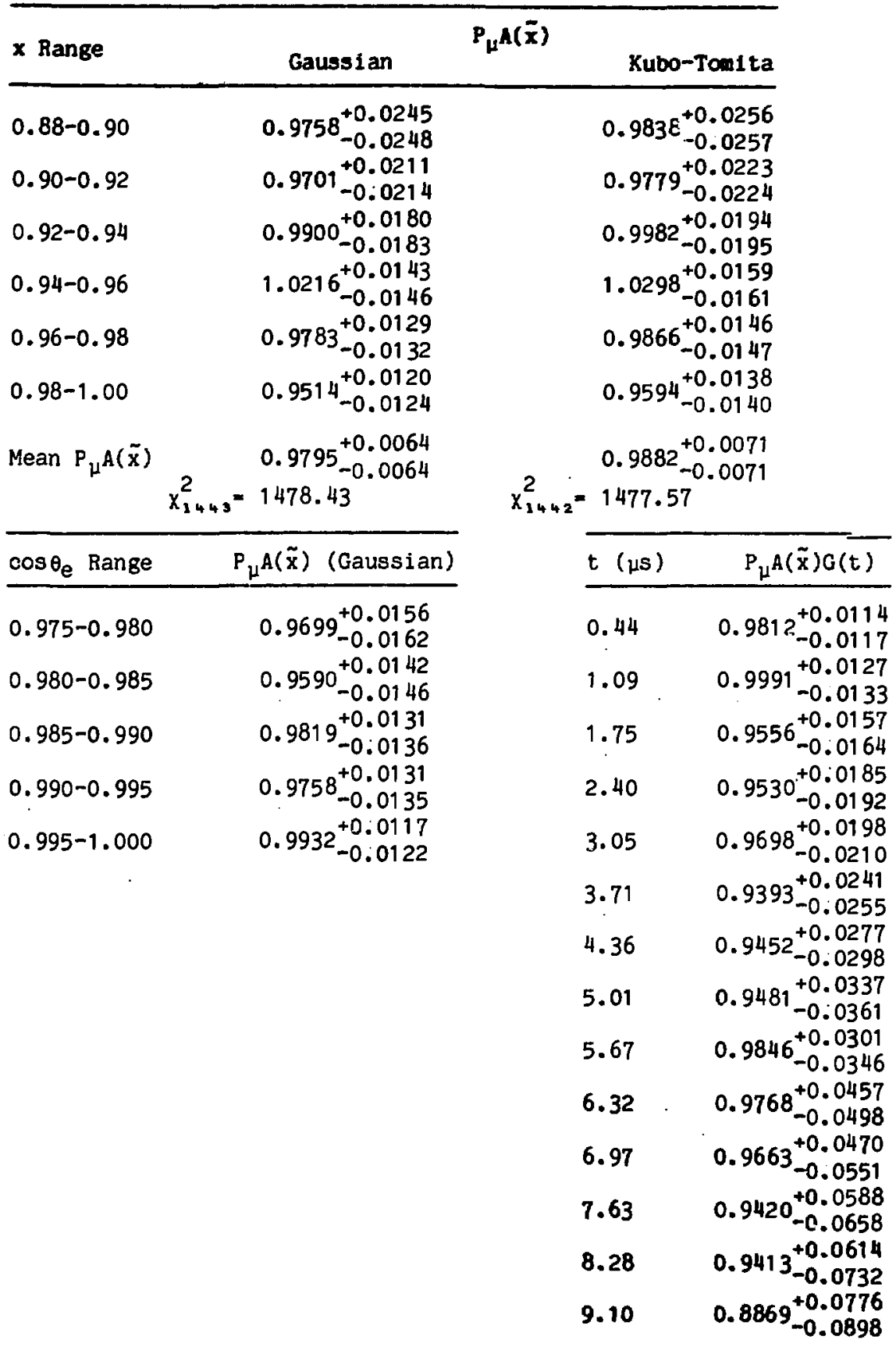

Table (C.1) cont. 
Run Period 2; Target $=\mathrm{Cu}^{*} ; \mathrm{B}_{\mathrm{T}}=110-\mathrm{G} ;$ Events fitted $=39244$

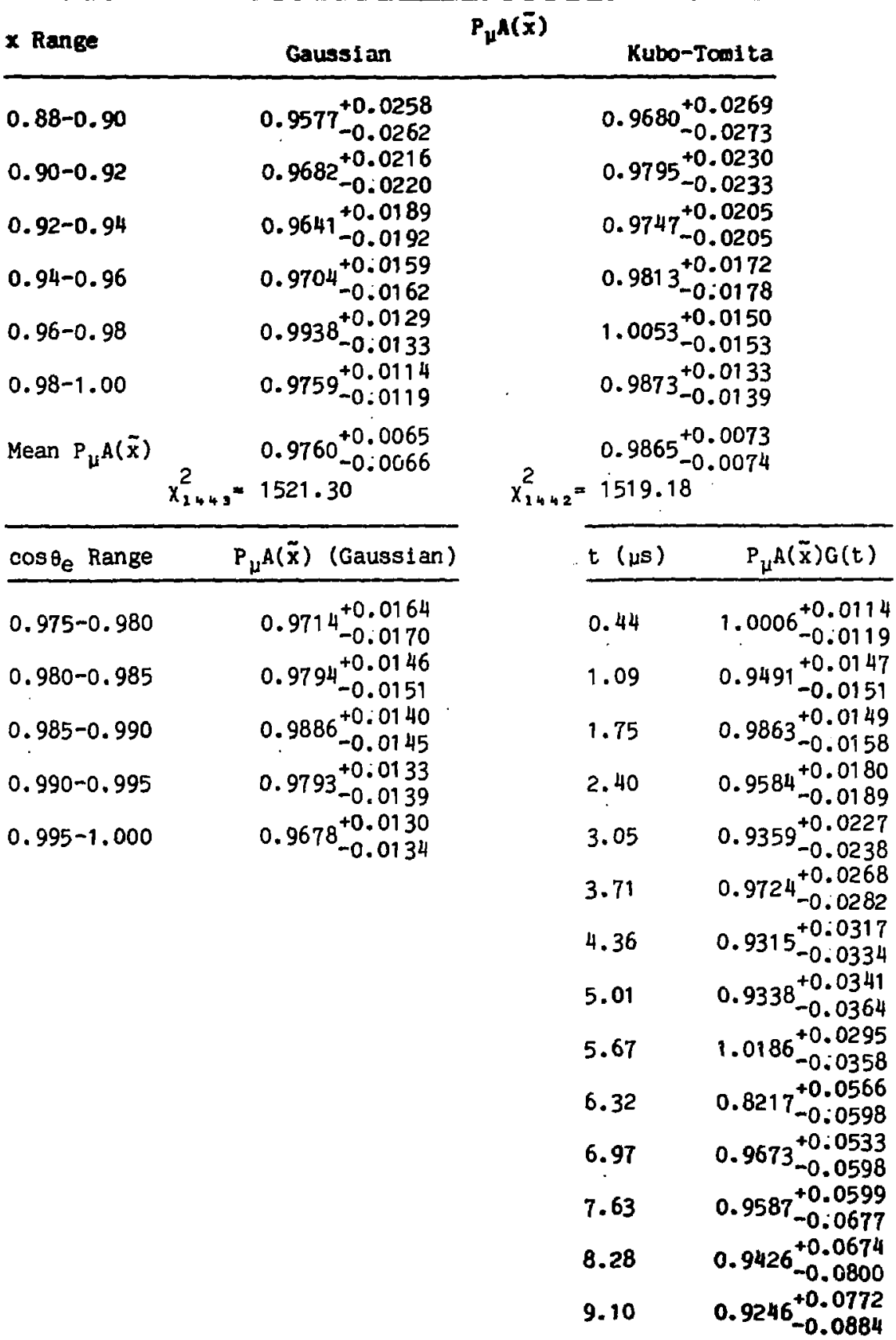

Table (C.1) cont. 
Run Perlod 3; Target $=$ A1; $B_{\mathrm{T}}=$ 110-G; Events Fitted $=98282$

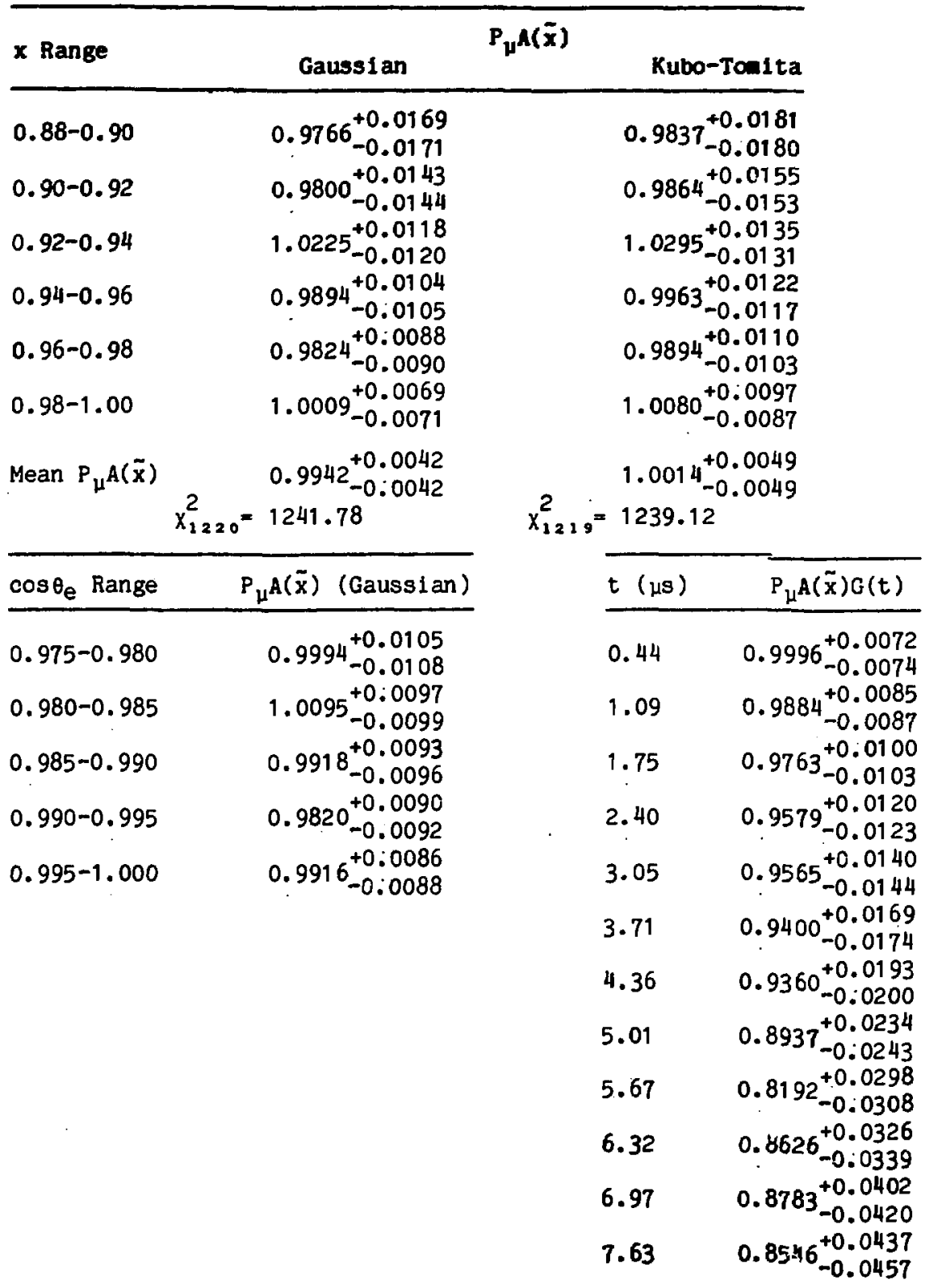




\begin{tabular}{|c|c|c|c|c|c|c|}
\hline $\begin{array}{l}\varepsilon 9 \varepsilon 0^{\circ} 0- \\
0 h 20^{\circ} 0- \\
1920^{\circ} 0- \\
2920^{\circ} 0- \\
L 2 \pitchfork 0^{\circ} 0- \\
1520^{\circ} 0- \\
S \hbar 20^{\circ} 0- \\
2220^{\circ} 0- \\
\varepsilon \varepsilon 20^{\circ} 0-\end{array}$ & 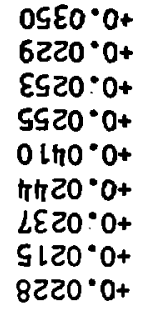 & $\begin{array}{l}82 L 8^{\circ} 0 \\
19 S 6^{\circ} 0 \\
S \pitchfork 98^{\circ} 0 \\
\angle 948^{\circ} 0 \\
6098^{\circ} 0 \\
S L S 8^{\circ} 0 \\
8 \& 88^{\circ} 0 \\
\pitchfork 268^{\circ} 0 \\
60 S 8^{\circ} 0\end{array}$ & 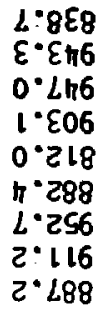 & $\begin{array}{l}O L \\
O L \\
O L \\
O L \\
O L \\
O L \\
O L \\
O L \\
O L\end{array}$ & 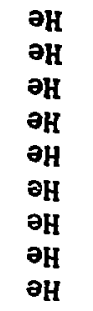 & $\begin{array}{l}29 \varepsilon \\
\text { } 2 \varepsilon \\
10 \varepsilon \\
1 S Z \\
\text { Shट } \\
1 \hbar 2 \\
0 \hbar 2 \\
\text { SE己 } \\
\varepsilon \varepsilon 己\end{array}$ \\
\hline $\begin{array}{l}\$ 810^{\circ} 0- \\
1020^{\circ} 0- \\
9610^{\circ} 0- \\
9410^{\circ} 0- \\
9610^{\circ} 0- \\
t 610^{\circ} 0- \\
6920^{\circ} 0-\end{array}$ & $\begin{array}{l}9 L 10^{\circ} 0+ \\
9610^{\circ} 0+ \\
0810^{\circ} 0+ \\
\text { LE } 10^{\circ} 0+ \\
h 810^{\circ} 0+ \\
0810^{\circ} 0+ \\
L \hbar 20^{\circ} 0+\end{array}$ & $\begin{array}{l}\varepsilon 200^{\circ} l \\
86860^{\circ} 0 \\
8 \varepsilon 00: 1 \\
89200^{\circ} l \\
2266: 0 \\
2266: 0 \\
6 \angle 00^{\circ} l\end{array}$ & $\begin{array}{l}L \cdot 6 \pitchfork 8 \\
2 \cdot 9 l 6 \\
6 \cdot 898 \\
6 \cdot 0 \varepsilon 6 \\
l \cdot 9 \varepsilon 6 \\
6 \cdot 0 L 8 \\
0 \cdot 298\end{array}$ & $\begin{array}{l}O L \\
O L \\
O L \\
O L \\
O L \\
O L \\
O L\end{array}$ & $\begin{array}{l}* \text { nj } \\
* \text { nj } \\
* \text { nj } \\
* n j \\
* n j \\
* n j \\
* n j\end{array}$ & $\begin{array}{l}\varepsilon \zeta \varepsilon \\
6 \varepsilon \varepsilon \\
\varepsilon \downarrow \varepsilon \\
L ટ Z \\
\varepsilon L Z \\
\zeta 0 Z \\
L 6 L\end{array}$ \\
\hline $\begin{array}{l}9810: 0- \\
Z L 10^{\circ} 0- \\
8020^{\circ} 0- \\
2910^{\circ} 0- \\
\varepsilon O Z 0: 0- \\
L E Z 0^{\circ} 0-\end{array}$ & $\begin{array}{l}E L L 0^{\circ} 0+ \\
L S 10^{\circ} 0+ \\
9810^{\circ} 0+ \\
6 \pitchfork 100^{\circ} 0+ \\
1610: 0+ \\
G 220^{\circ} 0+\end{array}$ & $\begin{array}{l}5900^{\circ} l \\
\angle 1 E 0^{\circ} l \\
6910^{\circ} l \\
\varepsilon 600^{\circ} l \\
\varepsilon 900^{\circ} l \\
6 r \hbar 6^{\circ} 0^{\circ}\end{array}$ & $\begin{array}{l}\hbar \cdot c 06 \\
\hbar: 806 \\
\varepsilon \cdot 9 L 6 \\
8 \cdot 9 L 6 \\
6 \cdot 056 \\
8 \cdot 968\end{array}$ & $\begin{array}{l}O L \\
0 L \\
O L \\
O L \\
O L \\
O L\end{array}$ & $\begin{array}{l}n y \\
n y \\
n y \\
n y \\
n y \\
n y\end{array}$ & $\begin{array}{l}8 \hbar \varepsilon \\
\hbar 2 \varepsilon \\
2 เ \varepsilon \\
0 Z 2 \\
012 \\
981\end{array}$ \\
\hline 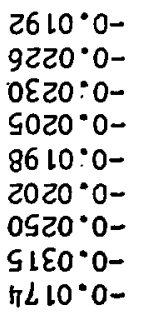 & $\begin{array}{l}8 L 10^{\circ} 0+ \\
\varepsilon 120^{\circ} 0+ \\
\mathcal{G} 120^{\circ} 0+ \\
2610: 0+ \\
0610^{\circ} 0+ \\
1610^{\circ} 0+ \\
2 \varepsilon 20^{\circ} 0+ \\
6820^{\circ} 0+ \\
1910^{\circ} 0+\end{array}$ & $\begin{array}{l}9986^{\circ} 0 \\
\varepsilon 096^{\circ} 0 \\
0686^{\circ}: 0 \\
8 L 00^{\circ} l \\
1 L 96^{\circ} 0 \\
9696^{\circ} 0 \\
\varepsilon L 86^{\circ}: 0 \\
6800^{\circ} \tau \\
6200^{\circ} \tau\end{array}$ & $\begin{array}{l}L \cdot 668 \\
S \cdot 068 \\
8 \cdot L 98 \\
Z \cdot \varepsilon 己 6 \\
6 \cdot L 46 \\
l \cdot 2 L 8 \\
l: 9 \varepsilon 8 \\
g^{*} \cdot 6 \varepsilon 8 \\
8^{-} \cdot 9 \pitchfork 8\end{array}$ & $\begin{array}{l}O L \\
O L \\
O L \\
0 L \\
0 L \\
O L \\
O L \\
O L \\
0 L\end{array}$ & $\begin{array}{l}\text { TH } \\
\text { TH } \\
\text { TH } \\
\text { TH } \\
\text { TH } \\
\text { TH } \\
\text { TH } \\
\text { TH } \\
\text { TY }\end{array}$ & $\begin{array}{l}L \hbar \varepsilon \\
L \downarrow \varepsilon \\
80 \varepsilon \\
26 己 \\
\hbar \varepsilon 己 \\
602 \\
002 \\
66 L \\
\varepsilon 8 l\end{array}$ \\
\hline $\begin{array}{l}\varsigma 910^{\circ} 0- \\
0610^{\circ} 0^{-} \\
1810^{\circ} 0- \\
8 L 10^{\circ} 0- \\
2020^{\circ} 0- \\
2610^{\circ} 0^{\circ-} \\
\varsigma \varepsilon 20^{\circ} 0^{-}\end{array}$ & $\begin{array}{l}\text { ES } 10^{\circ} 0+ \\
8 L 10^{\circ} 0+ \\
9 S 10^{\circ} 0+ \\
0 L 10^{\circ} 0+ \\
1610^{\circ} 0+ \\
8 L 10^{\circ} 0+ \\
\text { E120 }\end{array}$ & $\begin{array}{l}8510^{\circ} l \\
\angle 000^{\circ} l \\
h 8+0^{\circ} l \\
\varepsilon 100^{\circ} l \\
8216^{\circ} 0 \\
2200^{\circ} l \\
2 L 10^{\circ} l\end{array}$ & $\begin{array}{l}L \cdot 296 \\
6 \cdot \hbar 26 \\
\varepsilon \cdot L+18 \\
L \cdot \varepsilon 68 \\
L \cdot 016 \\
h \cdot 888 \\
L \cdot L 58\end{array}$ & $\begin{array}{l}O L \\
O L \\
O L \\
O L \\
O L \\
O L \\
O L\end{array}$ & $\begin{array}{l}8 y \\
8 y \\
8 y \\
8 y \\
8 y \\
8 y \\
8 y\end{array}$ & $\begin{array}{l}2 \zeta \varepsilon \\
0 \hbar \varepsilon \\
91 \varepsilon \\
822 \\
\hbar 12 \\
902 \\
161\end{array}$ \\
\hline Nowl & 17275 & $(x) u^{n d}$ & $\cos ^{6} x$ & to & 8021 & ung \\
\hline
\end{tabular}




\begin{tabular}{|c|c|c|c|c|c|c|}
\hline Run & Target & $\mathrm{B}_{\mathrm{T}}(\mathrm{G})$ & $x_{1431}^{2}$ & $P_{\mu} \Lambda(\tilde{x})$ & \multicolumn{2}{|c|}{ Statistical Error } \\
\hline 409 & Al & 70 & 1478.1 & 1.0126 & +0.0147 & -0.0154 \\
\hline 411 & $\mathrm{Al}$ & 70 & 1542.3 & 1.0186 & $\begin{array}{r}0.0145 \\
+0.014\end{array}$ & -0.0134 \\
\hline 419 & A1 & 70 & 1444.5 & 1.0138 & +0.0380 & -0.0151 \\
\hline 434 & Al & 70 & 1541.5 & $\begin{array}{r}1.0150 \\
0.9550\end{array}$ & +0.87 & -0.0191 \\
\hline 435 & Al & 70 & 1487.7 & 0.9559 & +0.0187 & -0.0193 \\
\hline 442 & Al & 70 & & 0.9794 & +0.0185 & -0.0192 \\
\hline 443 & Al & 70 & & 0.9723 & +0.0185 & -0.0192 \\
\hline 454 & Al & 70 & $\begin{array}{l}1530.0 \\
1514.8\end{array}$ & 0.9998 & +0.0193 & -0.0202 \\
\hline 468 & $\mathrm{Al}$ & 70 & $\begin{array}{r}1514.8 \\
.1544 .3\end{array}$ & 0.9789 & +0.0172 & -0.0180 \\
\hline 469 & $\mathrm{Al}$ & 70 & 1544.3 & 0.9801 & +0.0198 & -0.0207 \\
\hline 492 & $\mathrm{Al}$ & 70 & 1435.4 & 1.0254 & +0.0184 & -0.0194 \\
\hline 503 & A1 & 70 & 1515.0 & 0.9959 & +0.0133 & -0.0138 \\
\hline 504 & Al & $\begin{array}{l}70 \\
70\end{array}$ & 1499.9 & 0.9914 & +0.0149 & -0.0154 \\
\hline 517 & AI & 70 & $1394: 3$ & 0.9932 & +0.0140 & -0.0146 \\
\hline 518 & AI & 70 & 1512.4 & 1.0027 & +0.0153 & -0.0160 \\
\hline 529 & AL & 70 & 1494.6 & 0.9953 & +0.0182 & -0.0189 \\
\hline 530 & Al & 70 & 1557.4 & 1.0095 & +0.0173 & -0.0181 \\
\hline 541 & Al & 70 & 1535.2 & 0.9931 & +0.0167 & -0.0174 \\
\hline & Al & 70 & 1424.7 & 1.0091 & +0.0167 & -0.0174 \\
\hline & Al & 70 & 1507.2 & 1.0131 & +0.0175 & -0.0183 \\
\hline 249 & Al & 70 & 1489.3 & 0.9912 & +0.0171 & -0.0177 \\
\hline 550 & Al & 70 & 1544.7 & 0.9856 & $+0: 0171$ & -0.0181 \\
\hline 561 & Al & 70 & 1470.5 & 1.0068 & +0.0149 & -0.0156 \\
\hline 562 & Al & 70 & 1444.4 & 0.9723 & +0.0173 & -0.0180 \\
\hline 579 & Al & 110 & 1557.1 & 1.0074 & +0.0149 & -0.0159 \\
\hline 580 & Al & 110 & 1532.1 & $1: 0249$ & +0.0166 & -0.0173 \\
\hline 592 & Al & .110 & 1412.0 & 1.0186 & +0.0131 & -0.0141 \\
\hline 593 & Al & 110 & 1522.8 & 0.9752 & $+0: 0161$ & -0.0167 \\
\hline 619 & Al & 110 & 1489.9 & 0.9784 & +0.0181 & -0.0188 \\
\hline 620 & Al & 110 & 1373.0 & 0.9748 & +0.0190 & $-0: 0197$ \\
\hline 716 & Al & 110 & $1487: 7$ & 0.9887 & $+0: 0172$ & -0.0178 \\
\hline 717 & Al & 110 & 1479.2 & 1.0202 & +0.0149 & -0.0159 \\
\hline 723 & $A 1$ & 110 & 1474.6 & 0.9821 & $+0: 0174$ & -0.0182 \\
\hline 724 & Al & 110 & 1534.0 & 0.9948 & +0.0165 & -0.0173 \\
\hline 663 & $11 *$ & 110 & 1425.1 & 1.0222 & +0.0150 & -0.0163 \\
\hline 664 & A1* & 110 & 1502.8 & 0.9605 & +0.0185 & -0.0191 \\
\hline 673 & $A 1 *$ & 110 & $1464: 3$ & $1: 0014$ & $+0: 0160$ & -0.0170 \\
\hline 674 & $A 1 *$ & 110 & 1523.2 & 0.9804 & +0.0168 & -0.0175 \\
\hline 691 & $a 1 *$ & 110 & 1472.2 & $1: 0020$ & +0.0164 & -0.0172 \\
\hline 692 & $A 1 *$ & 110 & 1464.5 & 1.0148 & +0.0160 & -0.0170 \\
\hline 699 & $A L *$ & 110 & 1572.1 & 0.9673 & +0.0183 & -0.0189 \\
\hline 700 & $A I^{*}$ & 110 & 1440.6 & 0.9977 & +0.0174 & -0.0183 \\
\hline 707 & $A 1 *$ & 110 & 1518.7 & 1.0043 & $* 0.0159$ & -0.0167 \\
\hline 708 & $\boldsymbol{M} \mathbf{I}^{*}$ & 110 & 1549.9 & 0.9844 & $* 0.0160$ & -0.0167 \\
\hline
\end{tabular}




\begin{tabular}{|c|c|c|c|c|c|c|}
\hline Run & Target & $B_{T}(G)$ & $x_{1+51}^{2}$ & $P_{\mu} h(\tilde{x})$ & Statistica & al Error \\
\hline $\begin{array}{l}418 \\
430 \\
431 \\
446 \\
447 \\
472 \\
473 \\
495 \\
507 \\
508 \\
521 \\
522 \\
533 \\
534 \\
545 \\
546 \\
553 \\
554 \\
565 \\
566 \\
567\end{array}$ & $\begin{array}{l}A u \\
A u \\
A u \\
A u \\
A u \\
A u \\
A u \\
A u \\
A u \\
A u \\
A u \\
A u \\
A u \\
A u \\
A u \\
A u \\
A u \\
A u \\
A u \\
A u \\
A u\end{array}$ & $\begin{array}{l}70 \\
70 \\
70 \\
70 \\
70 \\
70 \\
70 \\
70 \\
70 \\
70 \\
70 \\
70 \\
70 \\
70 \\
70 \\
70 \\
70 \\
70 \\
70 \\
70 \\
70\end{array}$ & $\begin{array}{l}1390.3 \\
1409.2 \\
1445.2 \\
1443.8 \\
1510.7 \\
1425.3 \\
1421.5 \\
1576.2 \\
1399.5 \\
1474.7 \\
1401.2 \\
1454.3 \\
1414.5 \\
1412.4 \\
1495.5 \\
1529.2 \\
1412.5 \\
1461.5 \\
1538.2 \\
1410.1 \\
1273.9\end{array}$ & $\begin{array}{l}0.9686 \\
0.9790 \\
1.0137 \\
1.0001 \\
1.0090 \\
1.0137 \\
1.0138 \\
1.0020 \\
0.9933 \\
0.9937 \\
1.0115 \\
0.9890 \\
1.0116 \\
0.9682 \\
0.9877 \\
0.9927 \\
1.0017 \\
0.9759 \\
0.9798 \\
0.9999 \\
1.0264\end{array}$ & $\begin{array}{l}+0.0201 \\
+0.0156 \\
+0.0150 \\
+0.0162 \\
+0.0132 \\
+0.0175 \\
+0.0172 \\
+0.0143 \\
+0.0172 \\
+0.0150 \\
+0.0139 \\
+0.0148 \\
+0.0153 \\
+0.0183 \\
+0.0160 \\
+0.0153 \\
+0.0165 \\
+0.0176 \\
+0.0146 \\
+0.0170 \\
+0.0264\end{array}$ & $\begin{array}{l}-0.0210 \\
-0.0165 \\
-0.0162 \\
-0.0171 \\
-0.0143 \\
-0.0188 \\
-0.0189 \\
-0.0150 \\
-0.0182 \\
-0.0162 \\
-0.0147 \\
-0.0152 \\
-0.0165 \\
-0.0190 \\
-0.0168 \\
-0.0163 \\
-0.0174 \\
-0.0184 \\
-0.0152 \\
-0.0181 \\
-0.0289\end{array}$ \\
\hline $\begin{array}{l}583 \\
584 \\
596 \\
597\end{array}$ & $\begin{array}{l}\mathrm{Au} \\
\mathrm{Au} \\
\mathrm{Au} \\
\mathrm{Au}\end{array}$ & $\begin{array}{l}110 \\
110 \\
110 \\
110\end{array}$ & $\begin{array}{l}1535.3 \\
1485.9 \\
1512.4 \\
1448.6\end{array}$ & $\begin{array}{l}1.0254 \\
0.9834 \\
0.9910 \\
0.9742\end{array}$ & $\begin{array}{l}+0.0125 \\
+0.0147 \\
+0.0146 \\
+0.0146\end{array}$ & $\begin{array}{l}-0.0131 \\
-0.0152 \\
-0.0153 \\
-0.0152\end{array}$ \\
\hline $\begin{array}{l}414 \\
415 \\
426 \\
427 \\
440 \\
441 \\
450 \\
451 \\
464 \\
465 \\
487 \\
488 \\
4399 \\
499 \\
500 \\
513 \\
5 ! 4\end{array}$ & $\begin{array}{l}\mathrm{Cu} \\
\mathrm{Cu} \\
\mathrm{Cu} \\
\mathrm{Cu} \\
\mathrm{Cu} \\
\mathrm{Cu} \\
\mathrm{Cu} \\
\mathrm{Cu} \\
\mathrm{Cu} \\
\mathrm{Cu} \\
\mathrm{Cu} \\
\mathrm{Cu} \\
\mathrm{Cu} \\
\mathrm{Cu} \\
\mathrm{Cu} \\
\mathrm{Cu} \\
\mathrm{Cu}\end{array}$ & $\begin{array}{l}70 \\
70 \\
70 \\
70 \\
70 \\
70 \\
70 \\
70 \\
70 \\
70 \\
70 \\
70 \\
70 \\
70 \\
70 \\
70 \\
70\end{array}$ & $\begin{array}{l}1356.3 \\
1515.9 \\
1457.1 \\
1456.5 \\
1400.1 \\
1526.2 \\
1445.7 \\
1458.4 \\
1448.7 \\
1500.4 \\
1531.3 \\
1462.8 \\
1497.0 \\
1409.1 \\
1531.3 \\
1421.6 \\
1430.2\end{array}$ & $\begin{array}{l}0.9940 \\
0.9838 \\
0.9837 \\
0.9765 \\
0.9871 \\
0.9630 \\
0.9691 \\
0.9786 \\
0.9796 \\
0.9940 \\
1.0075 \\
0.9740 \\
0.9933 \\
0.9658 \\
0.9954 \\
0.9909 \\
0.9985\end{array}$ & $\begin{array}{l}+0.0219 \\
+0.0172 \\
+0.0169 \\
+0.0160 \\
+0.0163 \\
+0.0180 \\
+0.0187 \\
+0.0181 \\
+0.0166 \\
+0.0174 \\
+0.0117 \\
+0.0174 \\
+0.0138 \\
+0.0234 \\
+0.0154 \\
+0.0146 \\
+0.0143\end{array}$ & $\begin{array}{l}-0.0231 \\
-0.0180 \\
-0.0176 \\
-0.0167 \\
-0.0171 \\
-0.0187 \\
-0.0194 \\
-0.0189 \\
-0.0175 \\
-0.0185 \\
-0.0123 \\
-0.0182 \\
-0.0143 \\
-0.0247 \\
-0.0162 \\
-0.0152 \\
-0.0150\end{array}$ \\
\hline
\end{tabular}




\begin{tabular}{|c|c|c|c|c|c|c|}
\hline Run & Tar get & $B_{T}(G)$ & $x_{i+51}^{2}$ & $P_{\mu} A(\tilde{x})$ & Statistical & 11 Eiror \\
\hline $\begin{array}{l}525 \\
526 \\
537 \\
538 \\
557 \\
558\end{array}$ & $\begin{array}{l}\mathrm{Cu} \\
\mathrm{Cu} \\
\mathrm{Cu} \\
\mathrm{Cu} \\
\mathrm{Cu} \\
\mathrm{Cu}\end{array}$ & $\begin{array}{l}70 \\
70 \\
70 \\
70 \\
70 \\
70\end{array}$ & $\begin{array}{l}1437.6 \\
1458.8 \\
1412.3 \\
1511.5 \\
1473.8 \\
1474.8\end{array}$ & $\begin{array}{l}0.9686 \\
0.9755 \\
0.9984 \\
0.9830 \\
0.9769 \\
0.9945\end{array}$ & $\begin{array}{l}+0.0156 \\
+0.0185 \\
+0.0162 \\
+0.0169 \\
+0.0173 \\
+0.0169\end{array}$ & $\begin{array}{l}-0.0164 \\
-0.0192 \\
-0.0171 \\
-0.0178 \\
-0.0181 \\
-0.0175\end{array}$ \\
\hline $\begin{array}{l}575 \\
576 \\
588 \\
589 \\
712 \\
713\end{array}$ & $\begin{array}{l}\mathrm{Cu} \\
\mathrm{Cu} \\
\mathrm{Cu} \\
\mathrm{Cu} \\
\mathrm{Cu} \\
\mathrm{Cu}\end{array}$ & $\begin{array}{l}110 \\
110 \\
110 \\
110 \\
110 \\
110\end{array}$ & $\begin{array}{l}1520.0 \\
1612.0 \\
1442.1 \\
1531.7 \\
1446.6 \\
1503.2\end{array}$ & $\begin{array}{l}0.9920 \\
0.9629 \\
0.9903 \\
0.9711 \\
0.9705 \\
0.9587\end{array}$ & $\begin{array}{l}+0.0118 \\
+0.0165 \\
+0.0140 \\
+0.0144 \\
+0.0162 \\
+0.0166\end{array}$ & $\begin{array}{l}-0.0122 \\
-0.0171 \\
-0.0147 \\
-0.0149 \\
-0.0169 \\
-0.0172\end{array}$ \\
\hline $\begin{array}{l}600 \\
601 \\
669 \\
695 \\
696 \\
703 \\
704\end{array}$ & $\begin{array}{l}\mathrm{Cu}^{*} \\
\mathrm{Cu} \\
\mathrm{Cu} \\
\mathrm{Cu} \\
\mathrm{Cu}^{*} \\
\mathrm{Cu} \\
\mathrm{Cu}\end{array}$ & $\begin{array}{l}110 \\
110 \\
110 \\
11 J \\
1: 0 \\
110 \\
110\end{array}$ & $\begin{array}{l}1481.2 \\
1473.0 \\
1533.0 \\
1507.4 \\
1497.5 \\
1433.1 \\
1520.9\end{array}$ & $\begin{array}{l}0.9683 \\
0.9747 \\
0.9993 \\
0.9529 \\
0.9869 \\
0.9744 \\
0.9858\end{array}$ & $\begin{array}{l}+0.0158 \\
+0.0172 \\
+0.0159 \\
+0.0183 \\
+0.0182 \\
+0.0156 \\
+0.0163\end{array}$ & $\begin{array}{l}-0.0164 \\
-0.0180 \\
-0.0167 \\
-0.0190 \\
-0.0189 \\
-0.0163 \\
-0.0170\end{array}$ \\
\hline
\end{tabular}

\begin{tabular}{|c|c|c|c|c|c|c|}
\hline Run & Target & ${ }^{3} \mathrm{~T}(\mathrm{G})$ & $x_{1229}^{2}$ & $P_{\mu} A(\ddot{x})$ & Statistical & I Error \\
\hline $\begin{array}{l}883 \\
884 \\
890 \\
896 \\
903 \\
909 \\
914 \\
921 \\
928 \\
934 \\
940 \\
947\end{array}$ & $\begin{array}{l}\text { Al } \\
\text { Al } \\
\text { Al } \\
\text { Al } \\
\text { Al } \\
\text { AI } \\
\text { AI } \\
\text { AI } \\
\text { AI } \\
\text { Al } \\
\text { AI } \\
\text { Al }\end{array}$ & $\begin{array}{l}110 \\
110 \\
110 \\
110 \\
110 \\
1: 0 \\
110 \\
1: 0 \\
110 \\
110 \\
110 \\
110\end{array}$ & $\begin{array}{l}1305.1 \\
1271.9 \\
1353.9 \\
1400.9 \\
1325.0 \\
1303.9 \\
1285.5 \\
1197.7 \\
1357.0 \\
1359.3 \\
1346.3 \\
1363.6\end{array}$ & $\begin{array}{l}1.0018 \\
0.9703 \\
1.0101 \\
1.0033 \\
1.0038 \\
0.9300 \\
1.0112 \\
0.9859 \\
1.0074 \\
0.9908 \\
0.9957 \\
0.9520\end{array}$ & $\begin{array}{l}+0.0175 \\
+0.0153 \\
+0.0144 \\
+0.0122 \\
+0.0150 \\
+0.0140 \\
+0.0149 \\
+0.0163 \\
+0.0128 \\
+0.0126 \\
+0.0135 \\
+0.0149\end{array}$ & $\begin{array}{l}-0.0183 \\
-0.0158 \\
-0.0151 \\
-0.0139 \\
-0.0156 \\
-0.0145 \\
-0.0155 \\
-0.0169 \\
-0.0133 \\
.0 .0131 \\
-0.0140 \\
-0.0153\end{array}$ \\
\hline
\end{tabular}

Table (C.2) cont. 


\section{References}

1. S.L. Glashow, Nucl. Phys. 22, 579 (1961).

2. S. Heinber 8, Phys. Rev. Lett. 19, 1264 (1967).

3. A. Salam, Proc. $8^{\text {th }}$ Nobel Symposium, Aspenasgarden (Almgrist and Wiskell, Stockholm, 1968).

4. E.M. Lipmanov, Yad. Fiz. 6, 541 (1967) [Sov. J. Nucl. Phys. 6, 395 (1968)].

5. J.C. Pati and A. Salam, Phys. Rev. Lett. 31, 661 (1973) and Phys. Rev. D10, 275 (1974).

6. R.N. Mohapatra and J.C. Pati, Phys. Rev. D11, 566, 2588 (1975).

7. G. Senjanovic and R.N. Mohapatra, Phys. Rev. D12, 1502 (1975).

8. A.E. Pifer et al., Nucl. Instr. Meth. 135, 39 (1976).

9. J. Carr et al., Phys. Rev. Lett. 51, 627 (1983).

10. G. Arnison et al., Phys. Lett. 122B, 103 (1983) and 126B, 398 (1983).

11. M. Banner et al., Phys. Lett. 122B, 476 (1983).

12. W.J. Marciano and A. Sirlin, Phys. Rev. D29, 945 (1984).

13. G. Senjanovic, Nuc1. Phys. B153, 334 (1979).

14. M. Gell-Mann, P. Ramond and R. Slansky, unpublished.

15. R.N. Mohapatra and G. Senjanovic, Phys. Rev. Lett. 44. 912 (1980) and Phys. Rev. D23, 165 (1981).

16. H. Abramowicz et al.. í. Phys. C12, 225 (1982).

17. Primary Inpit to the worid average is V.V. Akhmanov et al., Yad. Fiz. 6, 316 (1967) [Sov. J. Nucl. Phys. 6, 230 (1968)].

18. Primary input to the world average is $\mathrm{J}$. Peoples, Nevis Cyclotron Report No. $147(1966)$. 
19. ¿. Yan XIInken, Mucl. Phys. 15, 145 (1966).

20. J. Van X11nken et al.. Phys. Rev. Lett. 50, 94 (1983).

21. D. Schreiber and F.T. Calaprice, pri vate cominication;

D. Schreiber, Ph.D. thesis, Princeton University, 1983

(unpublished); F.T. Calaprice et al., Phys. Rev. Lett. 35, 1566 (1975).

22. T. Vitale et al. (unpublished) quoted by B.R. Holstein and S.B. Treiman, Phys. Rev. D16, 2369 (1977).

23. L. Holfenstein, Phys. Rev. D29, 2130 (1984).

24. J. Donahue and B. Holstein, Phys. Lett 113B, 382 (1982). See also I.I. Bigi and J.M. Frere, Phys. Lett. 110B, 25E (1982).

25. G. Beall et al., Phys, Rev. Let.t. 48,848 (1982).

26. F. J. Gilman and M.H. Reno, Phys. Lett. 127B, 426 (1983).

27. P. DeForcrand, Ph.D. thes1s, UnIv. of Calif., Berkeley (1982), Lawrence Berkeley Laboratory Report No. LBL-14692.

28. T.D. Lee and C.N. Yang, Phys. Rev, 108, 1611 (1957).

29. F. Scheck, Phys, Lett. C44, 187 (1978).

30. A.M. Sachs and A. Sirlin, in Muon Physics, Vol. II, V. Hughes and C.S. Wu, eds. (Acadernic Press, N.Y., 1975), p.50.

31. C.G. Wohl et al. (Particle Data Group), Rev. Mod. Phys. 56, S1 (1984). Primary Input to $p$ is Ref. 18; primary input to $n$ is

F. Corriveau et al., Phys. Lett. 129B, 260 (1983) and S.E. Derenzo, Phys.Rev. 181, 1854 (1969); primary input to $E P_{\mu}$ is Rer. 17; primary input to $\xi \mathrm{P}_{\mu} \delta / p$ is ?et. 9; primary input to $\delta$ is D. Fryber ger, Phys. Rev. 166, 1379 (1968).

32. M.A.B. Beg et al., Phys. Rev. Lett. 38, 1252 (1977).

33. H.E. Fischer and F. Scheck, Nucl. Phys. 883, 25 (1974). 
34. V. Florescu and O. Kanei, hwovo ciments 611, 967 (1968).

35. M.A.B. Beg and A. Sirlin, Ann. Rev. Nucl. Scl. 24, 579 (7974).

36. K. Mursula and F. Scheck, iucl. Phys. B253, 189 (:925).

37. J.H. Brewer et al., in Mwon tuysics, VoI. III, V. Huetses ano

C.S. Wu eds. (Academic Press, N.Y., 1975), p.4.

38. G.W. Ford and C.J. Mullin, Phys. Rev. 108, 477 (1957).

39. v. Hughes, Phys. Rev. 108, 1106 (1957).

40. Proceedings of recent $\mu S R$ conferences are contaiced in Hyperfine Interactions 6 (1979) and 17-19 (1984).

41. D. Richter, in Neutron Scattering and Muon Spin Rotation, Springer Tracts in Modern Physies 101 (Springer-Veriag 1983) p. 85.

42. R. Kubo and K. Tomita, J. Phys. Soc. Jpn 9, 888 (1954).

43. J.H. van Vleck, Phys. Rev. 74, 1168 (1948).

44. K.W. Kehr et al., Z. Phys. B32, 49 (1973).

45. A.P. Mills, Proc. 6th Int. Conf. on Positron Annihilation, P.G. Coleman, S.C. Sharma, and L.M. Diana eds. (North-Holland, Amsterdam 1982) p.121.

46. C.J. Oram et al., Nucl. Instr. Meth. 179, 95 (198i).

47. R. Sagane et al., Phys. Rev. 95, 863 (1954).

48. K. Halbach, private communication.

49. Y.S. Tsai, SLAC-PUB-848 (1971).

50. B.P. N1gam et al., Phys. Rev. 115, 491 (1959).

51. J.B. Marion and B.A. Zimmerman, Nucl. Instr. Meth. 51, 93 (1967).

52. A.P. Rekalo, Ukr. Fiz, Zh, 16(12), 1937 (1971) and Yad, Fiz, 13(6), 1288 (1971) [Sov. J. Nucl. Phys. 13(6) 741 (1971)].

53. B. Balke et al., Laurence Berkeley Laboratory Report No. LBL-18320. 
54. M. Abolins et al., Proc. 1982 ofF Sumer Study Elen. Part. Pnjs. and Future Fac1lities p.274 (Snomiass, 1982).

55. E.J. Elchten et al.. Phys. Rev. Lett. 50, 811 (1983).

56. M.E. Peskin, private comunication.

57. K.D. Lane and E. Barany, private communication. 